\title{
Mechanisms for Enhanced Turbulence in the Drake Passage Region of the Southern Ocean \\ by
}

\author{
Sophia Tiare Merrifield \\ B.S., Yale University (2010) \\ Submitted to the Joint Program in Physical Oceanography \\ in partial fulfillment of the requirements for the degree of \\ Doctor of Philosophy \\ at the \\ MASSACHUSETTS INSTITUTE OF TECHNOLOGY \\ and the \\ WOODS HOLE OCEANOGRAPHIC INSTITUTION \\ June 2016 \\ (C)2016 Sophia T. Merrifield. \\ All rights reserved.
}

The author hereby grants to MIT and WHOI permission to reproduce and to distribute publicly paper and electronic copies of this thesis document in whole or in part in any medium now known or hereafter created.

Author

Joint Program in Physical Oceanography

Massachusetts Institute of Technology

\& Woods Hole Oceanographic Institution

May 4, 2016

Certified by

Louis St. Laurent

Senior Scientist

Woods Hole Oceanographic Institution

Thesis Supervisor

Certified by

W. Brechner Owens

Scientist Emeritus

Woods Hole Oceanographic Institution

Thesis Supervisor

Accepted by

Larry Pratt

Chairman, Joint Committee for Physical Oceanography Massachusetts Institute of Technology

Woods Hole Oceanographic Institution 


\title{
Mechanisms for Enhanced Turbulence in the Drake Passage \\ Region of the Southern Ocean
}

by

\author{
Sophia Tiare Merrifield \\ Submitted to the Joint Program in Physical Oceanography \\ Massachusetts Institute of Technology \\ \& Woods Hole Oceanographic Institution \\ on May 4, 2016, in partial fulfillment of the \\ requirements for the degree of \\ Doctor of Philosophy
}

\begin{abstract}
The Southern Ocean is one of the most energetic regions of the world ocean due to intense winds and storm forcing, strong currents in the form of the Antarctic Circumpolar Current (ACC) interacting with steep topography, and enhanced mesoscale activity. Consequently, the Southern Ocean is believed to be a hotspot for enhanced oceanic mixing. Due to the remote location and harsh conditions, few direct measurements of turbulence have been collected in the Southern Ocean. Previous studies have used indirect methods based on finestructure observations to suggest that strong mixing is ubiquitous below the mixed layer. Results from a US/UK field program, however, showed that enhanced internal wave finestructure and turbulence levels are not widespread, but limited to frontal zones where strong bottom currents collide with steep, large amplitude topography.

This thesis studies the processes that support turbulence and mixing in the surface boundary layer and at intermediate depths in the Drake Passage region. Direct measurements of turbulence show that previous estimates of mixing rates in the upper $1 \mathrm{~km}$ are biased high by up to two orders of magnitude. These biases are discussed in the context of the internal wave environment and enhanced thermohaline finestructure. The dissipation rate of thermal variance is enhanced in the upper $1000 \mathrm{~m}$, with the highest values found in northern Drake Passage where water mass variability is the most pronounced. Double diffusive processes and turbulence both contribute to buoyancy flux, elevating the effective mixing efficiency above the canonical value of 0.2 in the upper $1 \mathrm{~km}$. Despite the prevalence of energetic wind events, turbulence driven by downward propagating near-inertial wave shear is weak below the mixed layer. The results of this study inform large-scale modeling efforts through parameterizations of mixing processes in the highly undersampled Southern Ocean.
\end{abstract}

Thesis Supervisor: Louis St. Laurent

Title: Senior Scientist

Woods Hole Oceanographic Institution 
Thesis Supervisor: W. Brechner Owens

Title: Scientist Emeritus

Woods Hole Oceanographic Institution 


\section{Acknowledgments}

I would like to thank my advisors, Dr. Lou St. Laurent and Dr. Breck Owens, for their support and guidance in both academics and life beyond. I first met Lou in June of 2010, he gave me scopolamine patches and was incredulous to find out I had never seen Star Wars or really any of the culturally important movies. However, he seemed to understand my sense of humor and respect my taste in music. The years that followed were full of vibrant discussions covering science and the superiority of Generation X. As Lou always says, 'we run a cowboy operation', but I have learned so much from him, he is creative, careful and brilliant - he helped me immensely to develop my own compass as a scientist. I am grateful for his unrelenting belief in me and for the adventures collecting datasets in difficult conditions. I thank Breck for his patience and support. We spent many hours together at the blackboard dissecting problems from the ground up. He helped me to articulate and organize my ideas, and he was always a kind and patient ear, regardless of the topic.

I benefited significantly from interactions with my commitee and other mentors. Professor Raffaele Ferrari is an intellectual wonder, every conversation with him is fruitful and inspired. I am lucky to have had his ideas and guidance for the past 6 years. I thank Elena for cat-sitting and great soccer matches. Dr. Tom Farrar provided his valuable expertise and enthusiasm for upper ocean datasets. Dr. Andreas Thurhnerr shared his impressive knowledge and curiosity of the finescale and his Spanish language skills in South America. Dr. John Toole provided his continued interest and expertise in Drake Passage interleaving. Dr. Jake Gebbie was a friend, a fellow soccer enthusiast, and a source of sound advice. I thank Dr. Steve Jayne for his mentorship, friendship, and for his endearing sarcasm.

Jessica Kozik and Sean Whelan were heroic in our lab operations, data collection, and have become dear friends. Andy Miller steadied the ship whenever I couldn't, I am so grateful for his friendship. Tom Miller and Brian Green were always there for moral support in any form, elaborate brunches to mini golf. I spent many hours at the field and on the court, and it was there that I kept perspective and found some of my most valuable friends. I have many others to thank, including Colin, Becca, Melissa, Dan, Deepak, Gualtiero, Sam, Alec, Elena, Stephen, Mike, Kate and Jamie. Outside of the scientific world, I am indebted to Nicole, Laurel, and Claire who were strong pillars of strength and joy in my life.

I was raised in Hawaii by two oceanographers, it is no coincidence I couldn't find any more enjoyable use of my physics degree. From an early age my mother was determined to teach us math, along with many other things about life. She is a powerhouse of a woman and I will never stop learning from her, math or otherwise. My sister Anna is fascinating and supportive, the best sister I could come up with. She provides perspective and organizational skills when I need them most. My father is the stability in all of our lives, his presence and patience are incomparable. I owe my tinkering skills, and my sense of humor, to him. Finally, I dedicate this thesis to my grandfathers, both of whom I greatly admired. 
My time in the Joint Program was funded by the National Science Foundation through OCE-1232962. 


\section{Contents}

1 Introduction $\quad 19$

1.1 Southern Ocean processes . . . . . . . . . . . . . . . 22

1.2 Ocean mixed layer . . . . . . . . . . . . . . . . . . . . . 22

1.3 Stratified interior . . . . . . . . . . . . . . . . . . . 24

1.3.1 Lee-waves . . . . . . . . . . . . . . . . . . 25

1.3.2 Near-inertial waves . . . . . . . . . . . . . . . . . . 27

1.3.3 Double-diffusion . . . . . . . . . . . . . . . . 27

1.4 Measurement techniques . . . . . . . . . . . . . . 28

1.5 Observations . . . . . . . . . . . . . . . . 30

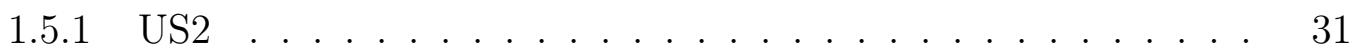

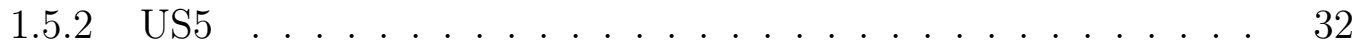

1.6 Outline . . . . . . . . . . . . . . . . . 37

2 Biases between finestructure and microstructure based mixing estimates $\quad 39$

2.1 Introduction . . . . . . . . . . . . . . . . . . 39

2.1.1 Estimates based on finestructure shear . . . . . . . . . . . . . 40

2.1.2 Estimates based on finestructure hydrography . . . . . . . . . 43

2.2 Data ........................... . . . 45

2.3 Results . . . . . . . . . . . . . . . . . . . . . 45

2.3.1 Strain parameterization . . . . . . . . . . . 48

2.4 Discussion . . . . . . . . . . . . . . . . . . . 52

3 Enhanced diapycnal diffusivity in intrusive regions 55

3.1 Introduction . . . . . . . . . . . . . . . . . 55

3.2 Water mass variability and $\mathrm{T} / \mathrm{S}$ instrusions . . . . . . . . . . . 58

3.3 Turbulent quantities in the upper $1500 \mathrm{~m}$. . . . . . . . . . . . 59

3.4 Statistics of dissipation at mid-depth sorted by density ratio and Richardson number . . . . . . . . . . . . . . . . 61

3.5 Methods of estimating vertical diffusivity . . . . . . . . . . . . 61

3.6 Spatial patterns in intrusive regions . . . . . . . . . . . . . 64

3.7 Discussion . . . . . . . . . . . . . . . . . . . 65

4 Mixed layer processes and boundary layer turbulence $\quad 73$ 
4.1 Introduction . . . . . . . . . . . . . . . . . . . 73

4.2 Mixed layer structure . . . . . . . . . . . . . . . . . . 75

4.2 .1 Hydrographic mixed layers . . . . . . . . . . . . . . 75

4.2 .2 Storm case studies . . . . . . . . . . . . . . . . . . . . 79

4.3 Boundary layer scalings . . . . . . . . . . . . . . . . . . . . . . . . . . 82

4.4 Discussion . . . . . . . . . . . . . . . . . . . . . . . 85

4.4.1 Langmuir turbulence . . . . . . . . . . . . . . 87

4.5 Appendix . . . . . . . . . . . . . . . . . . . . . . . . . . . 89

4.5 .1 Submesoscale instabilities . . . . . . . . . . . . . . 89

5 Near-inertial energy and turbulence in the wake of storms 91

5.1 Introduction . . . . . . . . . . . . . . . . . . . 91

5.2 Near-inertial energy flux to mixed layer motions . . . . . . . . . . . 93

5.2.1 Estimate from surface drifters and shipboard winds . . . . . 95

5.2 .2 Slab model . . . . . . . . . . . . . . . . . . . . 96

5.2 .3 Reanalysis wind products . . . . . . . . . . . . . . 101

5.3 Kinetic energy and dissipation . . . . . . . . . . . . . . . . . 104

5.4 Discussion . . . . . . . . . . . . . . . . . . . . 110

6 Discussion and Conclusions 113

6.1 Dynamical regimes in the Southern Ocean . . . . . . . . . . . . 113

6.2 Mixing efficiency . . . . . . . . . . . . . . . . . . . . . 113

6.3 Autonomous sampling strategies . . . . . . . . . . . . . . 116

6.4 Summary . . . . . . . . . . . . . . . . . . . . . . 118

$\begin{array}{ll}\text { A Appendix } & 121\end{array}$

A.0.1 Estimates and uncertainty associated with $\epsilon \ldots \ldots \ldots . \ldots 121$

A.0.2 Estimates and uncertainty associated with $\chi \ldots \ldots \ldots \ldots$ 


\section{List of Figures}

1-1 Schematic of the global overturning circulation from Lumpkin and Speer (2007). The Southern Ocean connects the three major ocean basins. . . . . . . . . . . . . . . . . . .

1-2 Schematic taken from Marshall and Speer (2012) representing the Upper and Lower Cell's of the MOC. The colors show zonally averaged oxygen values with high values (purple) showing recently ventilated water and low values (yellow) showing water that has not recently been ventilated. Internal mixing in the abyss is thought to add buoyancy to cause upwelling, as indicated by upward squiggly arrows. An additional upwelling pathway for NADW is adiabatic, involving advection southward toward the outcrop region of the Southern Ocean. . . . .

1-3 Schematic of important processes supporting turbulence and mixing in the Southern Ocean. Figure by Gualtiero Spiro Jaeger (MIT/WHOI $\mathrm{JP}) \ldots \ldots \ldots \ldots \ldots \ldots \ldots$

1-4 Images of the wave field during a storm off the Southern coast of Chile aboard the R/V Nathaniel B. Palmer. . . . . . . . . . . . . 23

1-5 Diagram describing Langmuir circulation from Thorpe (2004). . . . . 24

1-6 a) Schematic of the lee wave generation mechanism as a flow, $U$, interacts with topographic feature with wavelength $2 \pi / l$. b) Waves can be generated with the scale of the topography. They propagate upwards obeying a radiation condition with their ray path set in part by the local stratification, $N$. Ultimately these waves dissipate in the internal wave field at some distance downstream and aloft. (Adapted from Cushman-Roisin (1994)) . . . . . . . . . . . . . .

1-7 a) A parcel absorbs heat by diffusion and overshoots on vertical ascent leading to an oscillatory instability. b) A parcel equilibrates thermally but is still salty and therefore falls under gravity. . . . . . . . .

1-8 Piezoelectric air-foil shear probe created by T.R. Osborn. Figure from Lueck (2002). . . . . . . . . . . . . . . . . . . .

1-9 DIMES sampling, 2009-2014. Cruises which included microstructure measurements are shown in colors, deep microstructure profiles (DMP, x's) and CTD stations (o's). The water depth is shown in gray scale Smith and Sandwell (1997). The experiment sampled heavily in Drake Passage, with average winds exceeding 20 knots on most expeditions. Expeditions listed in white are not shown. . . . . . . . . . . 
1-10 Map showing the microstructure stations (magenta $x$ ) and LADCP (white $o$ ) taken during the US2 cruise. The colors are water depth from Smith and Sandwell (1997). . . . . . . . . . . . . . . .

1-11 A Rockland Scientific deep microstructure profiler is deployed off the stern of the Nathaniel B. Palmer during a cruise in Drake Passage. . 33

1-12 Same as Fig: 1-10 but for US5. . . . . . . . . . . . . . . . . . 34

1-13 Air temperature, sea surface temperature, windspeed, sensible, latent, shortwave, longwave and net heat fluxes during the US5 field expedition. The ship occupied a localized sampling area encountering storm activity multiple times. . . . . . . . . . . . . . . . .

1-14 Trajectories of 4 pairs of drifters deployed during the DIMES US5 cruise (Oct 2013). The distance between each pair is shown in the lower panel show for a 4 month period. The drifters are part of the Surface Velocity Program (SVP) with a $15 \mathrm{~m}$ drogue. . . . . . . . .

2-1 Comparison of dissipation rate and diffusivity estimates from two studies that examined turbulence and mixing levels along the 'Albatross' section crossing Drake Passage. The estimates of Naveira Garabato et al. (2004) used LADCP data and the Polzin et al. (2002) shearbased parameterization. The DIMES estimates were based on shear microstructure collected in 2010 (St. Laurent et al. (2012)). . . . . . .

2-2 Comparison of turbulent dissipation and and diffusivity estimated by Thompson et al. (2007) (black) to estimates from DIMES (blue) in the same region. . . . . . . . . . . . . . . . .

2-3 Map of CTD/LADCP and VMP stations taken during US5 overlaid on water depth from Smith and Sandwell (1997). The location of the time series stations and the number of casts delineated by the white boxes. . . . . . . . . . . . . . . . .

2-4 Potential temperature versus salinity for measurements made during the US5 expedition. The black line contours overlaid are potential density in units of $\mathrm{kg} / \mathrm{m}^{3}$. . . . . . . . . . . . . . .

2-5 PDF's of $\epsilon$ and $\chi$ in the near-surface, interleaving, and weakly stratified depth regimes relative to the full PDF over all depths. There is a distinct elevation in $\chi$ in the interleaving region where $T / S$ variance is significant. The dark purple color represents an overlap in the two PDF's. . . . . . . . . . . . . . . . .

2-6 Microstructure estimates of dissipation (blue) compared to finestructure strain estimates (red). There are significant discrepancies between the two methods at all depths, with the largest signal between 500$1500 \mathrm{~m}$. Estimates of $\epsilon_{\text {fine }}$ are shown for values of $R_{w}=3$ and 7 . .

2-7 Profiles of temperature, salinity, potential density and buoyancy frequency from a 16 hour time series. A profile with density step like features near $600 \mathrm{~m}$ is shown in the thick blue line. The thick black line represents the background stratification in the fourth panel. . . . . 
3-1 Map showing the microstructure stations taken during the US2 (blue) and US5 (red) cruises. The gray contours show water depth from Smith and Sandwell (1997). The white lines represent the climatological location of the Subantarctic and Polar Fronts, from Orsi et al. (1995).

3-2 Blue (US2, Jan-Mar 2010) and red (US5 Oct-Nov 2013) stations within $14 \mathrm{~km}$ of each other. Profiles of temperature (b) and salinity (c) show evidence of intrusive behavior between $300-1000 \mathrm{~m}$. The stratification (d) is similar in each season with the exception of a peak at the base of the mixed layer in Austral summer (Dec-Mar). Turbulent dissipation rates, $\epsilon(\mathrm{e})$ and $\chi(\mathrm{f})$ show similar seasonal characteristics in magnitude and depth structure. $\chi$ is enhanced in the regions of strong $\mathrm{T} / \mathrm{S}$ interleaving. The density ratio, $R_{\rho}(\mathrm{g})$ shows values in the intrusive region of the water column consistent with both diffusive convection and salt fingering. A closer look at the temperature profiles (h) shows regions where diffusive convection is likely occuring (black), and similarly for salt-fingering (green). . . . . . . . . . . . . . .

3-3 Averaged $\epsilon$ (a) and $\chi(\mathrm{b})$ as a function of depth. The colors represent different subsets of the data: US2 is split into upstream (SE Pacific) stations (black) and stations in Drake Passage (blue). Data from US5 are shown in red. The shaded regions represent error bars using a bootstrap method. The upper $200 \mathrm{~m}$ are not shown to remove the mixed layer and transition layer signal where stratification is highly variable and surface forcing has a significant impact. Averages are computed in 100 meter bins with stations whose bottom depth was shallower than $2000 \mathrm{~m}$ removed.

3-4 Probability density functions (PDFs) of $\epsilon(\mathrm{a}, \mathrm{b})$ and $\chi(\mathrm{c}, \mathrm{d})$ between 200-1000m are sorted by density ratio and Richardson number. Statistics in blue represent a stable density ratio $\left(R_{\rho}<0\right)$, and those in red represent a regime strongly susceptible to diffusive convection and salt fingering $\left(0.5<R_{\rho}<2\right)$. Statistics of $\epsilon$ are similar for all values of $R_{\rho}$. PDFs show preference to high values of $\chi$ for $R_{\rho}$ in the double diffusive regime. . . . . . . . . . . . . . . . .

3-5 Diffusivity as a function of depth computed using a) all data during the two expeditions and b) only data in the doubly stable regime, where $R_{\rho}<0$. The data are averaged in $100 \mathrm{~m}$ bins using the Osborn (1980) method $\left(\kappa_{\rho}\right.$, blue, a and b) and the Osborn and Cox (1972) method $\left(\kappa_{T}\right.$, red, a and $\left.\mathrm{b}\right)$. In the depth range associated with $\mathrm{T} / \mathrm{S}$ intrusions (400-800m), the values of $\kappa_{T}$ exceed $\kappa_{\rho}$ when all data are used. When data in the intrusive regime but with a doubly stable density ratio are used to compute diffusivity, this signal is eliminated and $\kappa_{T} \approx \kappa_{\rho}$. The ratio of c) $\kappa_{T} / \kappa_{\rho}$ as a function of depth is shown for the doubly stable regime (solid) and all data (dashed). . . . . . . . . . . . . . 
3-6 Three meridional transects of neutral density (colors) at a) $93^{\circ} \mathrm{W}$ b) $66^{\circ} \mathrm{W}$ and c) $65^{\circ} \mathrm{W}$. The circles represent regions in the water column where the density ratio $R_{\rho}$ is between $0.5-1$ (black) and 1-2 (white). These ranges represent potential for double-diffusive instabilities with growth rates comparable to the buoyancy frequency $(\mathrm{N})$, the timescale associated with internal wave-driven turbulence. The density ratio is preferable to double-diffusive instability in neutral density classes between 27.2 and $27.7 \mathrm{~kg} / \mathrm{m}^{3}$. The Polar Front in each panel is defined by the hydrographic measurements made during the respective cruises, US2 (a and b), US5 (c). . . . . . . . . . . . . . . .

3-7 The values of $\kappa_{\rho}$ (blue) and $\kappa_{T}$ (red) in $0.05 \mathrm{~kg} / \mathrm{m}^{3}$ neutral density bins using the US5 dataset. A linear regression over each intrusion is used to compute values of $\left|T_{z}\right|$ and $N^{2}$. The density is first sorted to the minimum potential energy state before calculating the buoyancy frequency. Only bins of neutral density, $\gamma$, that included intrusions are shown and the values of $\kappa_{T}$ and $\kappa_{\rho}$ are computed using intrusionscale quantities averaged in each bin. Additionally, only intrusions that satisfy the criterion of $0.5<R_{\rho}<2$ are shown to isolate the signal of features that are potentially doubly-diffusive. . . . . . . . . .

3-8 Ensemble averages in $0.05 \mathrm{~kg} / \mathrm{m}^{3}$ neutral density bins are taken of $\langle\chi\rangle / 2$ and $\left\langle\left|\nabla_{\text {iso }} T\right|\right\rangle^{2}$ to study the limit of the thermal variance budget where vertical dissipation is neglected. In this limit, the value of $K_{e}$, taken as upper bound, is generally larger than $10^{3} \mathrm{~m}^{2} / \mathrm{s}$. The black lines represent lines of constant $K_{e}$ of $10^{2}, 10^{3}$, and $10^{4} \mathrm{~m}^{2} / \mathrm{s} \ldots \ldots$

3-9 Values of the dissipation ratio, $\Gamma$, using intrusion scale quanitites that satisfy $0.5<R_{\rho}<2$. The values are consistently higher than 0.2 in the intrusive regime. . . . . . . . . . . .

4-1 Seasonal mean mixed layer depth from the 2010 climatology (Holte and Roemmich (2010)). Pink dots represent the location of DIMES microstructure measurements. All All DIMES data except US5 (OctNov) were collected in Austral Summer (January-March). . . . . . . .

4-2 Structure of temperature, salinity and potential density as a function of season in Drake Passage. The US2 survey (blue) extended further south in Drake Passage relative to the US5 survey (Red) which was localized in the northern region. . . . . . . . . . . . . .

4-3 The wind speed (a) as a function of time during the US2 deployment coincident to Shear (b), buoyancy frequency (c), and dissipation (d). The seasonal stratification at $75-150 \mathrm{~m}$ exceeds the shear acting to suppress turbulence at the base of the mixed layer. . . . . . . . . . .

4-4 Structure of temperature (upper row) and TKE dissipation rate (lower row) for the 5 VMP yo-yo sections taken during a storm. High values of TKE dissipation rate show the same structure as temperature suggesting an actively mixing layer. The very near surface shows large values of $\epsilon$ most likely associated with breaking surface waves. . . . . 
4-5 The a) wind stress b) latent and sensible heat fluxes c) shortwave and longwave heat fluxes d) total heat flux e) buoyancy flux and f) MoninObukhov length during the US5 field program. . . . . . . . . .

4-6 Near surface pressure from NASA MERRA during the US5 expedition. 82

4-7 Measurements of dissipation (gray) and temperature (black) offset to show the structure of the boundary layer during the second storm (beginning on Nov. 6). The boundary layer deepened in the first three profiles before a capping layer developed as the heat flux changed to stabilizing. The red dots represent values of $\epsilon_{s}$, the Monin-Obukhov scaling in the wind dominated regime. The blue line shows the value of the buoyancy flux, $J_{b}$, during the destabilizing phase. . . . . . . .

4-8 Depth structure of measured dissipation from 5 tow-yo transects during US5. The black line represents the transect mean, while the red and blue are the Monin-Obukhov scalings of wind stress, $\epsilon_{s}$ (red), and buoyancy flux, $J_{b}$ (blue). The values of the Obukhov length are given in the title, showing that $\zeta<<1$ throughout the the measurements. This suggests wind-stress dominates turbulent production. . . . . . .

4-9 Vertical structure of scaled dissipation as a function of depth normalized by the mixed layer depth. Averages are taken along each transect. Figure follows Lombardo and Gregg (1989) Fig. 11. . . . . . . . . .

4-10 The wind speed averaged over 2 hours prior to each microstructure measurement is shown relative to the mixing depth, $H_{\epsilon}$, calculated as the depth where $\epsilon$ decays to $10^{-9} \mathrm{~W} / \mathrm{kg}$. . . . . . . . . . . . .

4-11 Actively mixing layer depth vs. the mixed layer depth where the colors represent the wind speed averaged 2 hours prior to the measurement of turbulence. The lines represent (from top to bottom), $H_{\epsilon} / H_{\rho}=1$, $H_{\epsilon} / H_{\rho}=0.5$ and $H_{\epsilon} / H_{\rho}=0.1 . \ldots . . . . . . .$.

4-12 a) Strong wind speed in the Southern Ocean, b) Stokes drift, and as a result the Langmuir number, $L a$ is often lower than 0.3 in the Southern Ocean. Figures adapted from Liu et al. (2014) . . . . . . . . . . .

5-1 Map of the 3 drifter pair trajectories colored by the distance from the ship. Inset is a map with the cruise track (white) and drifter tracks (magenta) overlayed on water depth. . . . . . . . . . . .

5-2 a) Rotary spectrum of a representative drifter velocity time series (D1) shows significant CCW energy at near-inertial frequencies. The thick solid vertical line denotes the mean inertial period over the course of the drifter deployment, the dashed line the M2 frequency, and the thin lines the $0.65-1.5 \mathrm{f}$ frequency band. The thin blue line is the spectra before de-tiding. b) The zonal current component $(\mathrm{u})$ estimated from drifter D1, compared with the near-inertial bandpass current $\left(u_{i}\right)$ and the TPXO tidal current $\left(u_{t}\right)$. c) Same as (b) for the meridional current

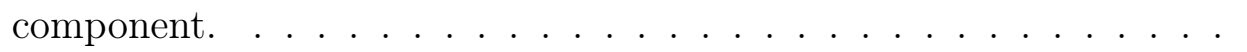


5-3 The (a) total and (b) inertial energy flux from the wind to mixed layer motions evaluated along the path of each drifter. c) Path of the six drifters with the distance between each drifter and the ship indicated in color. . . . . . . . . . . . . . . . . . .

5-4 Rotary frequency spectra of the velocity estimated from the slab model. 99

5-5 a) Zonal and b) meridional velocity (solid lines) estimated from the slab model forced with shipboard winds and a constant mixed layer $H=50 \mathrm{~m}$ and $r=0.15 \mathrm{f}$. The dashed line is the bandpass filtered near-inertial current which dominates the variance. . . . . . . . . . . 100

5-6 Similar to 5-5 but with $r=0.02 f$ to represent very weak damping. . 101

5-7 a) Zonal and b) meridional components of the wind vector measured from the ship (blue) and from the ECMWF reanalysis product (red). The wind product is well correlated to the record from the ship's mast. 102

5-8 Timeseries of inertial energy flux from three methods described in the text. The slab model forced with reanalysis winds gives the weakest near-inertial flux of the methods studied. . . . . . . . . . . . . .

5-9 Comparison of the decimated shipboard wind record to the ECMWF wind record where colors represent the difference in the inertial energy flux from the slab model between the two methods. The laregest discrepancies occur at the highest wind speeds. . . . . . . . . . .

5-10 a) AVISO geostrophic velocities vs. estimated geostrophic velocities computed by removing b) the equivalent barotropic vertical structure function, $\mu(z)$ from each profile. . . . . . . . . . . . .

5-11 The total (blue), residual (red) and inertial (green) velocities from a representative profile in the time series. The left panel shows the zonal component of the velocity and the right panel the meridional component. The inertial fit is estimated for two sets of timeseries stations during US5. The velocity components are fit to cosine and sine waves with inertial periods as a function of depth. . . . . . . .

5-12 Power spectra of vertical shear normalized by mean buoyancy frequency in $750 \mathrm{~m}$ vertical bins. The vertical columns are total energy $(\mathrm{a}, \mathrm{d}, \mathrm{g})$, counter-clockwise with depth $(\mathrm{b}, \mathrm{e}, \mathrm{h})$, and clockwise with depth $(c, f, i)$ separated by space (upstream vs. Drake Passage) and season (Austral summer vs. Austral spring). . . . . . . . . . . . . . 108

5-13 Inertial energy flux vs. integrated dissipation in the mixed layer (left panel) and at mid-depth (right panel). Integrated dissipation rates are large in the mixed layer, often exceeding values of $E_{i}$. Enhanced ageostrophic energy and dissipation are found at mid-depths during Austral spring in Drake Passage (red dots) and summer (blue dots). The weakest values are found upstream of the Passage in Austral summer (black dots). . . . . . . . . . . . . . . . 
5-14 PDF's of dissipation (a,b,c) in the 200-2000m range separated spatially and seasonally. Values of dissipation in the SE Pacific are nearbackground throughout the region below the mixed layer. The stratification profiles $(\mathrm{d}, \mathrm{e}, \mathrm{f})$ show a strongly stratified layer in Austral summer which had eroded during the Austral Spring field experiment. . . . . 110

6-1 Scatter plots of $R i_{g}$ vs. $R e_{b}$ in the a) interleaving regime (200-1000m) and $b)$ deep ocean $(z>1000 m)$. The data points are colored by the value of $\epsilon$ with values of $\epsilon<10^{-10}$ removed from the analysis. . . . . 114

6-2 The same as Fig. 6-1 except colors show the value of $\chi$. . . . . . . 115

6-3 Estimates of the dissipation ratio, $\Gamma$, from statistics of US5 below the interleaving features. The values are estimated for the upper quartile of the dataset based on values of $\epsilon$. . . . . . . . . . . . . . 116

A-1 Shear spectra as a function of pressure, black line represents the Nasmyth spectrum for a given value of $\epsilon$. The upper middle panel shows a case where one probe had an anomalous response, most likely due to impact with zoo plankton. In this case, only the spectral signal of the good probe is used for the estimate of $\epsilon$. . . . . . . . . . . . . 122 


\section{List of Tables}

1.1 DIMES cruises with microstructure measurements . . . . . . . . . . 31

5.1 Summary of methods used to estimate the inertial energy flux, $E_{i},{ }^{*} 2 \mathrm{~d}$ refers to the same wind record as method 2, but decimated to 6-hour temporal resolution. . . . . . . . . . . . . . . . . . . . 102 


\section{Chapter 1}

\section{Introduction}

Oceanic turbulence and mixing contribute significantly to the energetics and circulation of the global ocean. Turbulence results in the transfer of momentum and heat far exceeding those of molecular processes alone. Moreover, small-scale mixing is an important sink for kinetic energy in the interior ocean. At the largest scales, winds, tides, and solar forcing provide energy to the oceanic circulation. Through a variety of mechanisms, energy cascades downward to the smallest scales where it is dissipated by molecular viscosity into heat. These processes are well-studied but difficult to observe, such that closing the energy budget is still a fundamental open question in modern oceanography.

The Southern Ocean is a unique region of the global ocean. Strong westerly winds drive the Antarctic Circumpolar Current (ACC), an energetic current that flows unimpeded by geographical boundaries. The ACC connects the Indian, Atlantic and Pacific ocean basins as schematized by Lumpkin and Speer (2007) (Fig. 1-1). In this sense the Southern Ocean is a distinct branch of the Meridional Overturning Circulation (MOC). The MOC is responsible for the transport of heat from low to high latitudes and atmospheric carbon into the deep ocean. Knowledge of the spatial distribution of mixing rates plays a central role in our understanding of the MOC, and ultimately the climate system.

All models of the ocean's MOC are based on the assumption of the existence of a steady-state buoyancy balance of the deep water masses. The conceptual model for the buoyancy budget of the deep ocean interior was first formulated by Stommel and Arons (1960) and Munk (1966). The "Abyssal Recipes" study has been scrutinized 


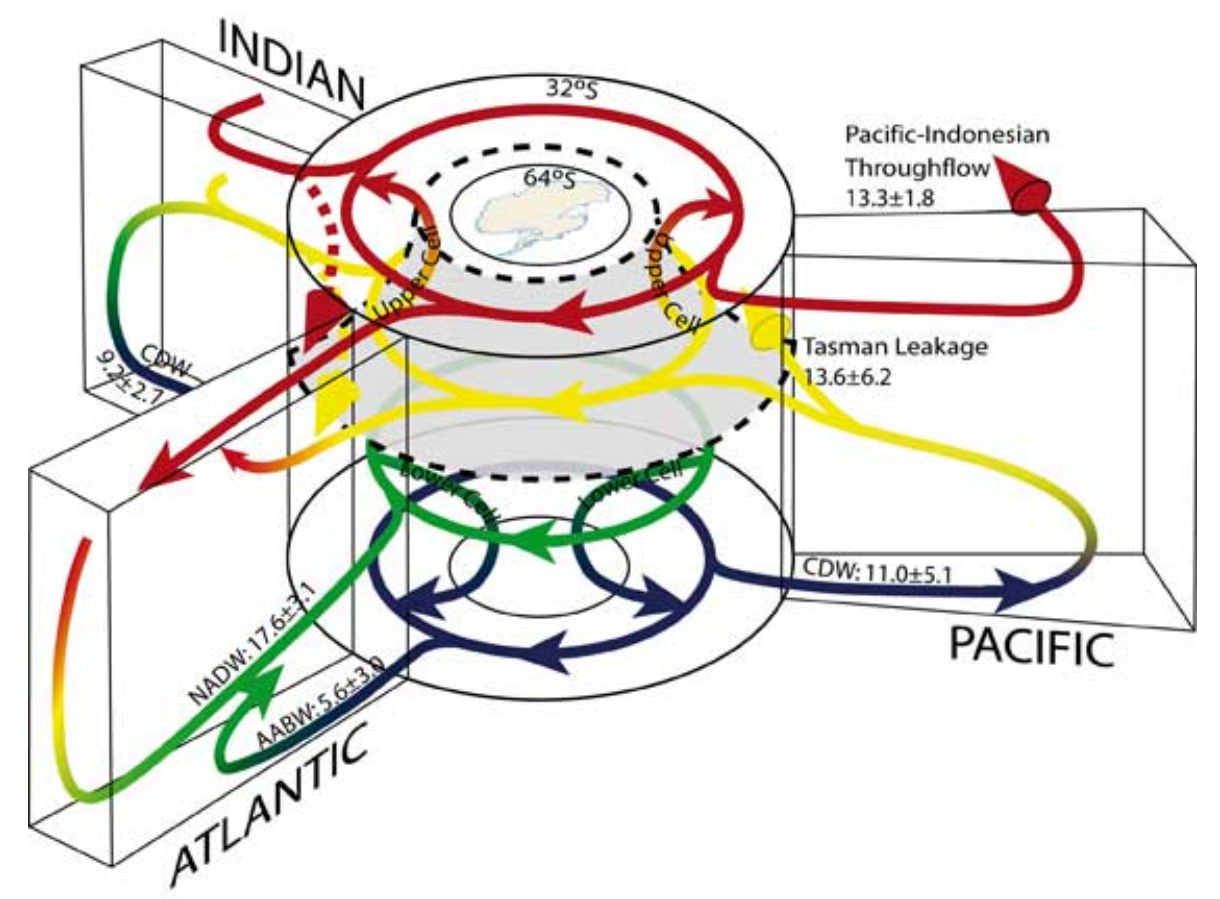

Figure 1-1: Schematic of the global overturning circulation from Lumpkin and Speer (2007). The Southern Ocean connects the three major ocean basins.

in more contemporary frameworks (Munk and Wunsch (1998), Wunsch and Ferrari (2004), St. Laurent and Simmons (2006)), but still stands as a pillar of our thermodynamic understanding of the large-scale deep circulation. Turbulent mixing has long been understood to provide the mechanism that transfers heat from the temperate waters of the upper ocean to the colder waters of the abyss. This heat transfer acts to modify the deep and bottom water masses of the abyss in the North Atlantic Deep Water (NADW) and Antarctic Bottom Water (AABW). The buoyancy exchanged by mixing results in a net upwelling of dense fluid within the abyssal ocean. In a globally averaged sense, this conversion and upwelling of abyssal water balances the production of deep water at high latitudes, allowing the ocean's density structure to remain in steady state.

The modern view of the MOC includes two meridional overturning cells as schematized by Marshall and Speer (2012) (Fig. 1-2). The upper cell is characterized by sinking dense waters in the northern North Atlantic and flow along isopycnals at mid-depth. In this view, the upper cell can be closed with only minimal mixing in the ocean interior (e.g. Sloyan and Rintoul (2001), Webb and Suginohara (2001)). The lower cell is characterized by dense water that forms near Antarctica and comes 
back toward the surface by crossing isopycnals as a consequence of the buoyancy gained through mixing. In this view, mixing in the ocean interior is thus only required for the densest waters of the lower cell. This Southern Ocean pathway has been demonstrated in the idealized numerical simulations of Toggweiler and Samuels (1998), Wolfe and Cessi (2010), and Nikurashin and Vallis (2011) and modelled in more realistic simulations by Marshall and Speer (2012).

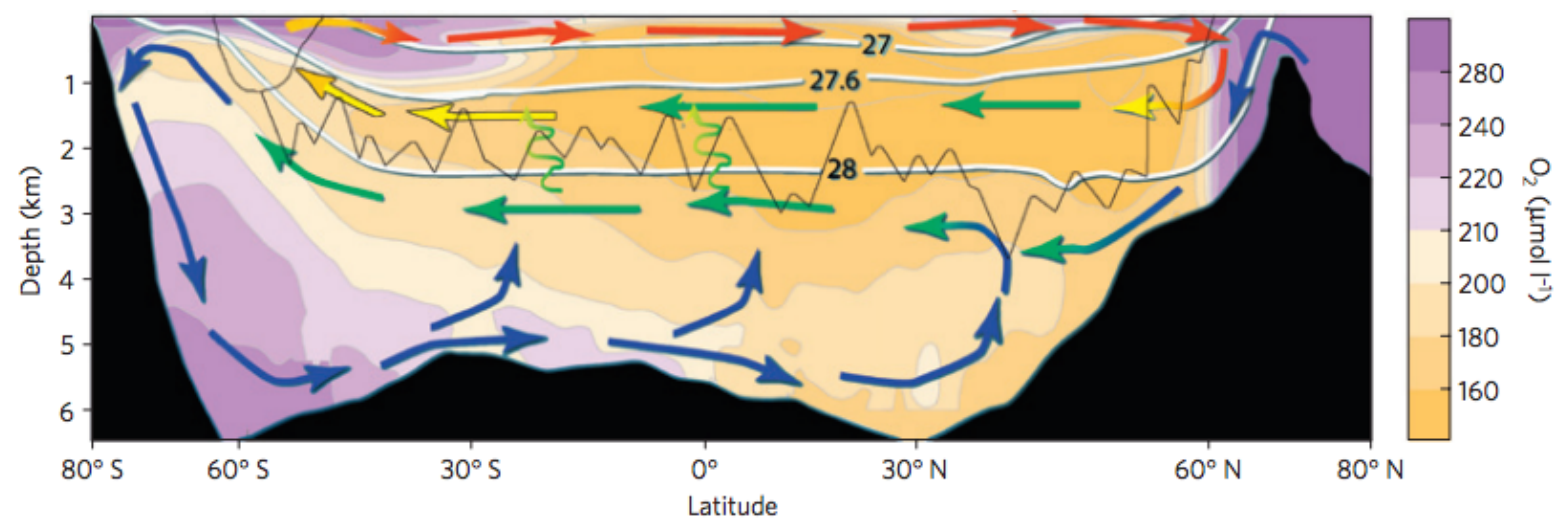

Figure 1-2: Schematic taken from Marshall and Speer (2012) representing the Upper and Lower Cell's of the MOC. The colors show zonally averaged oxygen values with high values (purple) showing recently ventilated water and low values (yellow) showing water that has not recently been ventilated. Internal mixing in the abyss is thought to add buoyancy to cause upwelling, as indicated by upward squiggly arrows. An additional upwelling pathway for NADW is adiabatic, involving advection southward toward the outcrop region of the Southern Ocean.

Observational support for the role of mixing in the lower and upper cells of the MOC remains elusive, especially in the remote Southern Ocean. It is still unclear whether diapycnal mixing is an order one process in the Southern Ocean, although observational studies have suggested that diapycnal mixing levels are very large in both the deep and shallow depths of the ACC (Naveira Garabato et al. (2004), Thompson et al. (2007)). The recently completed Diapycnal and Isopycnal Mixing Experiment in the Southern Ocean (DIMES) field program provides a comprehensive new dataset to study the role of mixing the Southern Ocean, and the processes that support it. In particular, as small-scale turbulent processes are not resolved in general ocean circulation models, physically based parameterizations determined from observational studies are the only means of introducing realistic mixing rates into simulations used to forecast the climate. 


\subsection{Southern Ocean processes}

There are a variety of processes that lead to turbulent mixing in the Southern Ocean. Internal waves are generated at both the sea surface and over varying bottom topography. Double-diffusive instabilities can develop in parts of the water column where watermass variability is pronounced. The mixed layer turbulence is generated from a combination of strong winds, convection, wave-driven processes such as Langmuir turbulence and overturning instabilities at small-scale fronts. This thesis explores the processes that support diapycnal mixing in the Southern Ocean (Fig. 1-3). A description of the relevant physics, with particular attention to the dominant processes in the Southern Ocean, follows.

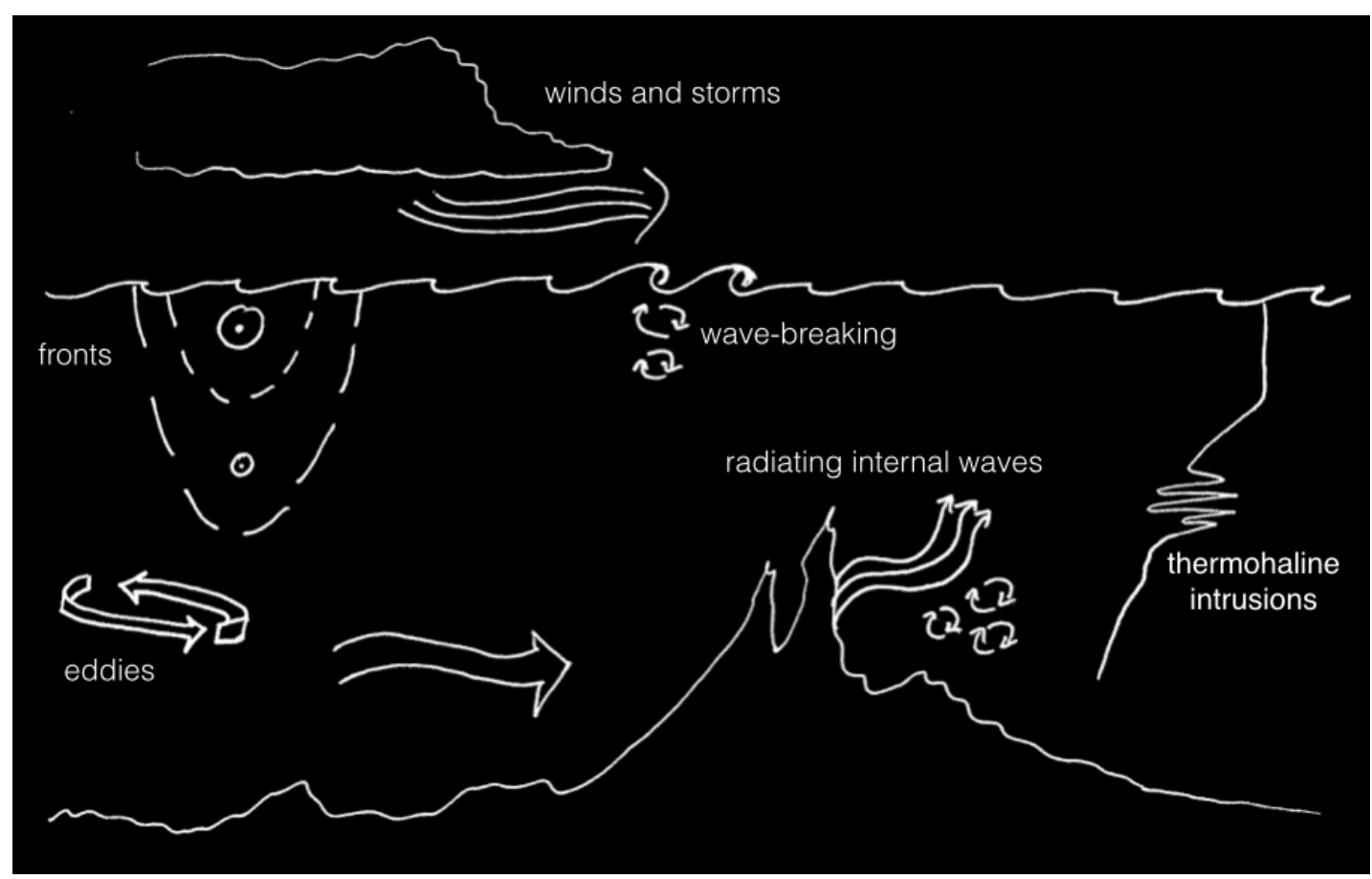

Figure 1-3: Schematic of important processes supporting turbulence and mixing in the Southern Ocean. Figure by Gualtiero Spiro Jaeger (MIT/WHOI JP).

\subsection{Ocean mixed layer}

Interactions between the atmosphere and ocean are crucial to the redistribution of heat and carbon dioxide. Water mass properties are modified at the atmosphere-ocean interface, resulting in new density classes. Outcropping isopycnals in the Southern 
Ocean provide a pathway for these surface properties to reach the interior ocean.

There are a variety of processes that support turbulent mixing in the oceanic surface boundary layer. At the surface, the mixed layer is driven by buoyancy fluxes and mechanical stress generated by the wind. Strong westerly winds and storms force the surface ocean at sub-inertial and near-inertial frequencies, where inertial refers to the local Coriolis frequency, $f$. Deep winter mixed layers driven by seasonal heat loss are characteristic of the region. Extreme wind and wave states (Fig. 1-4) suggest breaking waves are an important mechanism for enhanced dissipation in the near-surface layer.

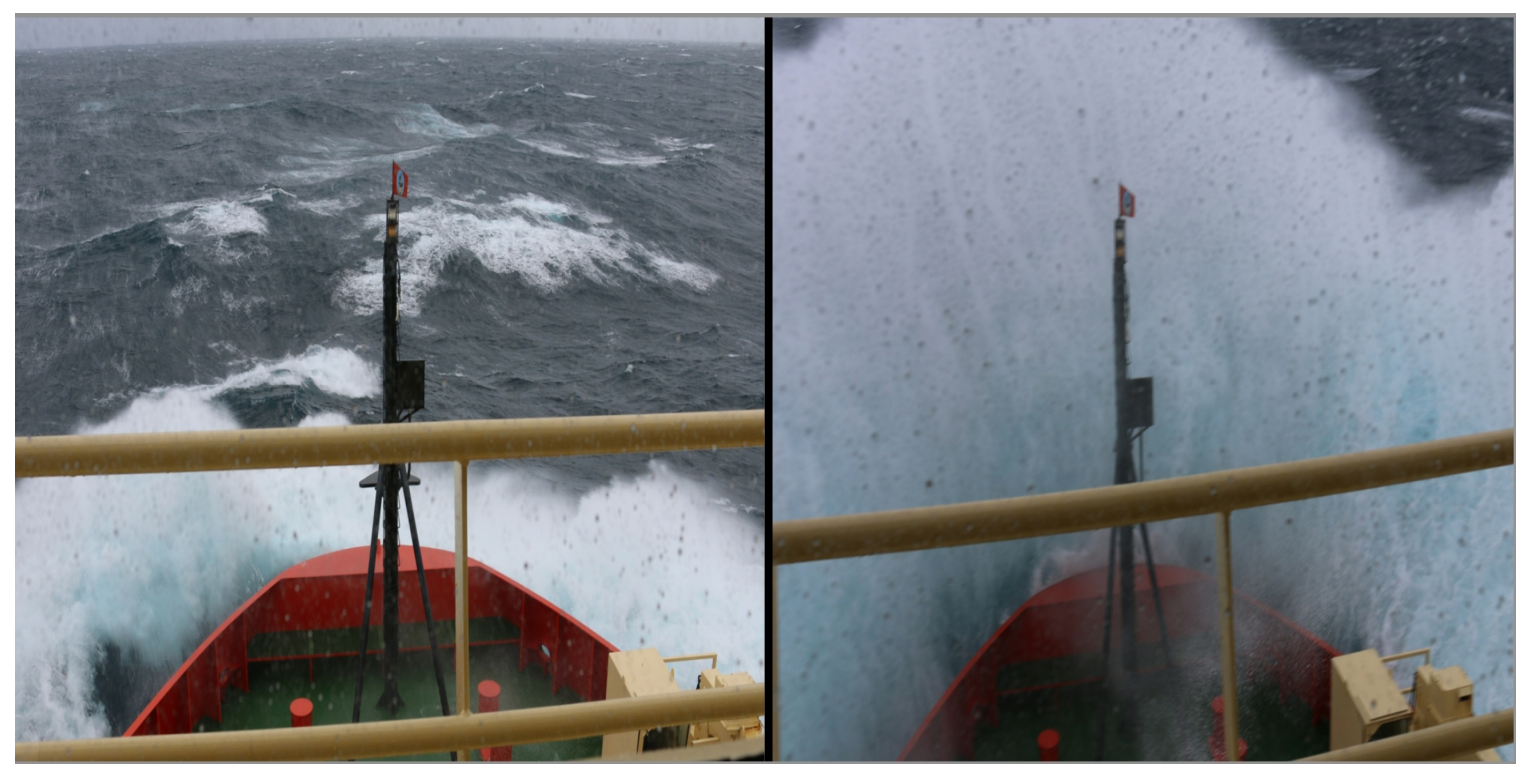

Figure 1-4: Images of the wave field during a storm off the Southern coast of Chile aboard the R/V Nathaniel B. Palmer.

In the boundary layer, the interaction between surface waves and ocean currents give rise to Langmuir circulations. Irvine Langmuir's observations in the 1920's of sargassum aligned in 'windrows' lead to a theory to explain the streaks (Langmuir et al. (1938), Leibovich (1983)). He proposed that counter-rotating vortices aligned with the wind likely contribute to maintaining the mixed layer (Fig. 1-5). The turbulent nature of these cells is an active area of research (e.g. Belcher et al. (2012), Li et al. (2015)). Notably, these cells can concentrate turbulent kinetic energy and deepen the boundary layer. It is believed that regions, such as the Southern Ocean, with strong, steady winds and an energtic surface wave field provide favorable conditions for Langmuir turbulence.

In addition to mixing driven by wind and buoyancy forcing at the surface, the bound- 


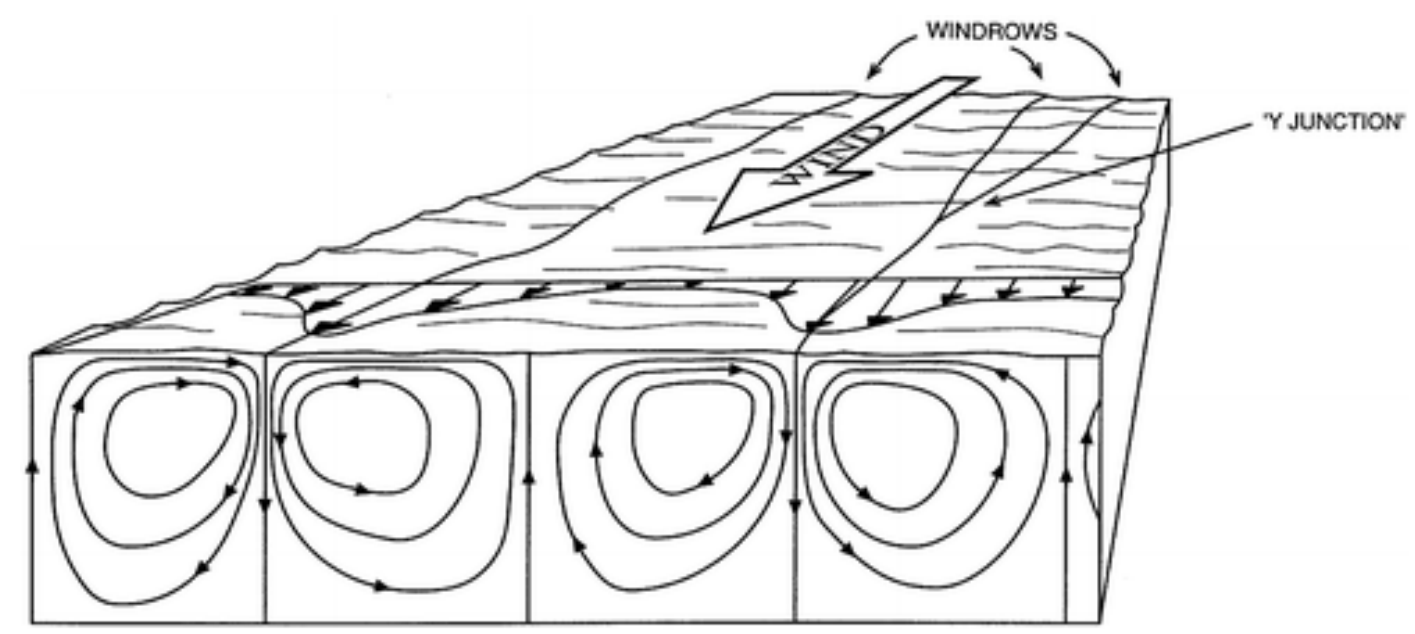

Figure 1-5: Diagram describing Langmuir circulation from Thorpe (2004).

ary layer is characterized by a rich submesoscale frontal field. These features are characterized by $O(1)$ Rossby number, $O(1 \mathrm{~km})$ spatial scales and $O(1$ day) timescales. Submesoscale instabilities are believed to lead to enhanced turbulent mixing that removes energy from the boundary layer (Thomas and Taylor (2010)). Examples of gravitational, symmetric, and centrifugal instability have been investigated in numerical simulations and observational campaigns (e.g. Callies et al. (2015), Thompson et al. (2016)). In chapter 4, we study the processes supporting mixed layer turbulent dissipation.

\subsection{Stratified interior}

Below the mixed layer, density stratification in the interior ocean supports internal waves with frequencies between the local Coriolis frequency, $f$, and the buoyancy frequency, $N$. Tidal currents are also contained within this frequency band. Sources for the internal waves are usually at large scales (inertial and tidal) and the broader spectrum is the result of wave-wave interactions. The relationship between the internal wave field and dissipation was emphasized by the seminal work of Munk (1966) which suggested that internal wave breaking was a significant source of ocean mixing away from boundaries. Internal wave breaking often occurs due to shear instabilities characterized by low Richardson numbers (the ratio of the buoyancy frequency to the square of the vertical velocity shear).

Internal waves can be generated at the surface through winds or from the bottom by 
geostrophic or tidal flows. In the Southern Ocean, tides are weak thus geostrophic motions dominate internal wave generation at the seafloor. Two classes of internal waves important to mixing and dissipation in the Southern Ocean are described in further detail.

\subsubsection{Lee-waves}

A well-studied feature of the Southern Ocean is the internal wave generation that results as the eddies and jets associated with the Antarctic Circumpolar Current (ACC) flows over topography. Since the advent of acoustic Doppler current profiling, measurements in the Southern Ocean have demonstrated internal wave velocity finestructure in regions of rough topography. This includes the Kerguelen Plateau (Polzin and Firing (1997), Waterman et al. (2014)) and the Scotia Sea (Naveira Garabato et al. (2004)). The presence of velocity, and in some cases, density finestructure has been widely interpreted as signatures of enhanced turbulence and mixing levels.

Geostrophic motions evolve on timescales greater than the local Coriolis frequency such that they can be considered stationary at leading order in wave radiation theory. The linearized governing equations for internal lee waves by steady flows are:

$$
\begin{array}{r}
U u_{x}+w U_{z}-f v=-p_{x} \\
U v_{x}+f u=0 \\
U w_{x}=-p_{z}+b \\
U b_{x}+N^{2} w=0 \\
u_{x}+w_{z}=0
\end{array}
$$

where $U=U(z)$ and $N$ are the parameters characterizing the background flow and stratification. At the lower boundary, the velocity perpendicular to topography must vanish which is equivalent to $w(x, 0)=U \frac{\partial h}{\partial x}$ where $h(x)$ is the topography. The system is considered to have no variations in the $y$-direction. Combining the equations into one for the vertical velocity, $w$, yields:

$$
U^{2}\left(w_{x x}+w_{z z}\right)_{x x}+N^{2} w_{x x}+f^{2} w_{z z}-U U_{z z} w_{x x}=0
$$

The intrinsic frequency, the frequency in the reference frame of the mean flow, of the 
waves is set by the topography and mean flow, ie $\omega=k U$. Assuming a plane wave solution, the relation for the vertical wavenumber, $m$, is given by

$$
m^{2}=\frac{k^{2}\left(N^{2}-k^{2} U^{2}\right)-k \omega U_{z z}}{\left(k^{2} U^{2}-f^{2}\right)}
$$

If the mean flow $\mathrm{U}$ is independent of depth, the relation reduces to, $m^{2}=k^{2}\left(N^{2}-\right.$ $\left.k^{2} U^{2}\right) /\left(k^{2} U^{2}-f^{2}\right)$.

Radiating internal lee waves are constrained by horizontal wavenumber according to $f / U<k<N / U$. Taking typical values of $f=1.2 \times 10^{-4} \mathrm{~s}^{-1}, N=10^{-3} \mathrm{~s}^{-1}$, and $U=10^{-1} \mathrm{~m} \mathrm{~s}^{-1}$, the lee wave band has horizontal wavelengths in the range of $500 \mathrm{~m}$ to $5 \mathrm{~km}$. Waves in this range have intrinsic frequencies $(\omega=k \cdot U)$ from $1.17 \cdot f$ to $7 \cdot f$.
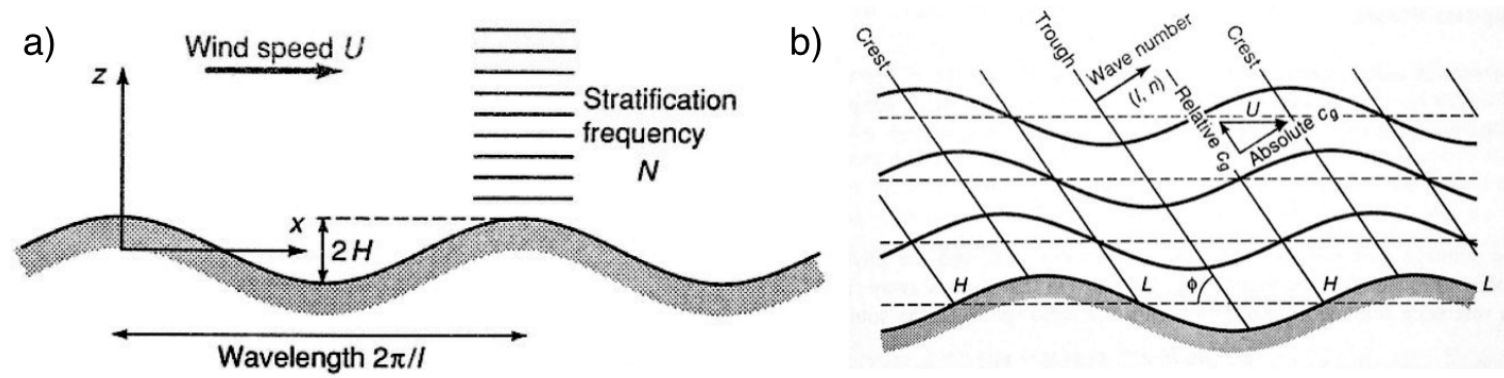

Figure 1-6: a) Schematic of the lee wave generation mechanism as a flow, $U$, interacts with topographic feature with wavelength $2 \pi / l$. b) Waves can be generated with the scale of the topography. They propagate upwards obeying a radiation condition with their ray path set in part by the local stratification, $N$. Ultimately these waves dissipate in the internal wave field at some distance downstream and aloft. (Adapted from Cushman-Roisin (1994))

The relationship between lee waves and turbulent mixing in the Southern Ocean is poorly constrained. The nonlocal effects of lee waves are particularly difficult to estimate from field observations. Studies based on indirect estimates of turbulence have suggested that mixing rates in the ACC range from modestly enhanced (Sloyan (2005), Kunze et al. (2006)) to extremely large (Naveira Garabato et al. (2004), Thompson et al. (2007)). Work based on finestructure has given ambiguous indications for the spatial distribution of enhanced turbulence. Studies by Sloyan (2005) and Kunze et al. (2006) suggest enhanced mixing rates occur local to topography in the frontal zones of the ACC. Naveira Garabato et al. (2004), on the other hand, characterized the occurrence of enhanced turbulence as "wide-spread," suggesting that internal wave 
processes somehow saturate the ACC region. Thompson et al. (2007) inferred very large mixing rates in the upper $1000 \mathrm{~m}$ downstream of the Phoenix Ridge/Shackleton Fracture Zone. In chapter 2 we discuss the use of finestructure parameterizations in the deep Southern Ocean where lee-wave activity is prominent. In chapter 5, we assess evidence for non-local lee-wave energy at mid-depths in the Drake Passage region.

\subsubsection{Near-inertial waves}

Intermittent wind events and storms lead to near-inertial motions in the mixed layer. After the passage of a storm, these currents undergo an adjustment generating internal waves with near-inertial frequencies. At frequencies close to $f$, internal wave currents are nearly horizontal. Observations reveal that enhanced variability near $f$ is ubiquitous in the global ocean. Near-inertial waves (NIWs) have short vertical scales and therefore are a source of enhanced turbulence in the interior ocean via shear instability.

The Southern Ocean has strong westerlies as well as mesoscale storms which provide time-variable winds and inertially rotating forcing to the system. Many studies have suggested that breaking NIWs generated by wind forcing contribute to mixing rates below the thermocline in the Southern Ocean (Thompson et al. (2007), Wu et al. (2011)). However, other studies aiming to quantify the flux of energy from the wind to near-inertial motions have found a global minima at $60^{\circ} \mathrm{S}$ (Alford (2003)). Due to a lack of in-situ measurements, the seasonality and storm response of the ocean in this region in the context of near-inertial variability are uncertain. In chapter 5, we use observations from the Drake Passage region of the Southern Ocean to address aspects of near-inertial energy and turbulence in the stratified interior.

\subsubsection{Double-diffusion}

In addition to internal wave breaking, an additional process that can drive mixing in the stratified interior is double-diffusive instability. A unique characteristic of the Southern Ocean is significant watermass variability and interleaving of temperature $(T)$ and salinity $(S)$. As a result, convective instability in these features is a potential source for enhanced mixing. Double-diffusion is a convective instability that occurs due to differing rates of molecular diffusion between heat and salt. This form of 
instability has implications for vertical fluxes of heat and salt in the ocean. The two forms of double-diffusive instability are diffusive convection, where cold and fresh water overlies warm and salty water, and salt fingers, which occurs in the reverse configuration (Fig. 1-7a and b, respectively).

a) diffusive convection COLD/FRESH
WARM/SALTY b) salt fingers

WARM/SALTY

COLD/FRESH

Figure 1-7: a) A parcel absorbs heat by diffusion and overshoots on vertical ascent leading to an oscillatory instability. b) A parcel equilibrates thermally but is still salty and therefore falls under gravity.

Double-diffusive instabilities are studied using the density ratio, $R_{\rho}=\frac{\alpha \bar{T}_{z}}{\beta \bar{S}_{z}}$, where $\alpha$ is the thermal expansion coefficient, $\beta$ is the haline contraction coefficient, $T_{z}$ is the vertical gradient of temperature, $S_{z}$ is the vertical gradient of salinity and the $\left(^{-}\right)$ operator refers to a background mean. The development of the instability can be described by a growth rate, $\lambda\left(R_{\rho}\right)$. Super-critical growing modes (Schmitt (1979)) develop when $\lambda\left(R_{\rho}\right)<2 \pi N^{-1}$, where $N$ is the local buoyancy frequency. $R_{\rho}$ can be estimated from hydrographic data. Double diffusion is observed typically in low energy regimes, where molecular diffusion is not overwhelmed by turbulent eddy fluxes. Despite energetic wind and buoyancy forcing, signatures of double diffusion have been observed in Southern Ocean frontal zones (Toole and Georgi (1981)). Further details of double-diffusive instablities in the Southern Ocean are described in chapter 3.

\subsection{Measurement techniques}

We review the techniques used to measure mixing in the stratified ocean. For decades, direct measurements of the turbulent dissipation, $\epsilon$, have been made with air-foil probes mounted on free-falling instruments (Osborn (1974)). The probes utilize a piezoelectric crystal covered in a rubber sheath (Fig. 1-8). Piezoelectric materials generate an electric charge in response to a mechanical force. As the profiler falls 
through the water, changes in the lateral water speed are measured by the probe and converted into a velocity shear. Turbulence instruments have the capability to detect values of $\epsilon=10^{-10} \mathrm{~W} / \mathrm{kg}($ Lueck (2002)).

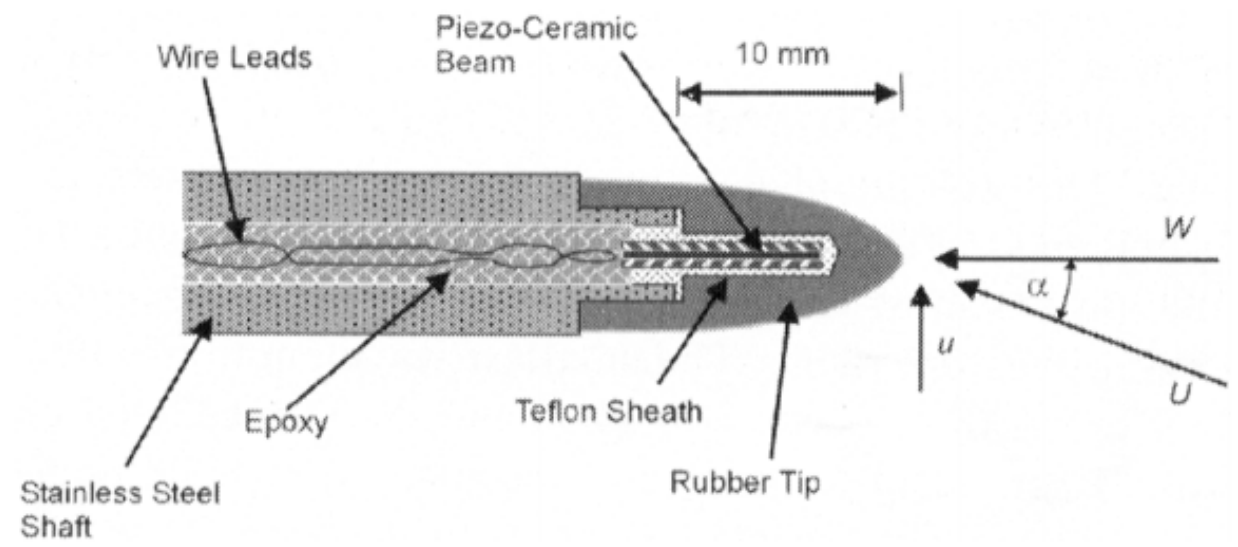

Figure 1-8: Piezoelectric air-foil shear probe created by T.R. Osborn. Figure from Lueck (2002).

In isotropic turbulence, the dissipation rate is related to the turbulent shear and the strain rate as

$$
\epsilon=\frac{15}{2} \nu \overline{\left(\frac{\partial u}{\partial z}\right)^{2}}=15 \nu \overline{\left(\frac{\partial u}{\partial x}\right)^{2}}
$$

where $\nu=10^{-6} \mathrm{~m}^{2} / \mathrm{s}$ is the kinematic moelcular viscosity, $\mathrm{u}$ is the velocity in the $\mathrm{x}$-direction, $\mathrm{z}$ is orthogonal to $\mathrm{x}$ and the overbar represents an ensemble average. The instrument must be able to measure the wavenumber range of microstructure velocity fluctuations. The scale at which motion is arrested and turbulent kinetic energy is dissipated into heat occurs near $\left(\nu^{3} / \epsilon\right)^{1 / 4}$. The microstructure range of velocity fluctuations is $0.23-11 \mathrm{cpm}$ in regions of the open ocean with low values of dissipation $\left(\epsilon \sim 10^{-10} \mathrm{~W} / \mathrm{kg}\right)$ and $2.3-110 \mathrm{cpm}$ in more energetic regions $\left(\epsilon \sim 10^{-6} \mathrm{~W} / \mathrm{kg}\right)$ (Lueck $(2002))$. These ranges put specific constraints on the fall-speed of the profiler and probe response. 


\subsection{Observations}

The Diapycnal and Isopycnal Mixing Experiment in the Southern Ocean (DIMES) recorded more than 500 hydrographic and 200 turbulence full-depth profiles with heavy sampling in Drake Passage (Fig 1-9, Table 1.1). This field program, and the findings that have resulted from it, have improved our understanding of the role of the Southern Ocean in the climate system. In particular, the first direct measurements of turbulent dissipation and mixing rates in the Southern Ocean were made as part of the DIMES study. Ledwell et al. (2010), St. Laurent et al. (2012), and Sheen et al. (2014) showed that enhanced internal wave fine-structure and turbulence levels were not widespread, but limited to frontal zones where strong bottom currents collide with steep large-amplitude topography.

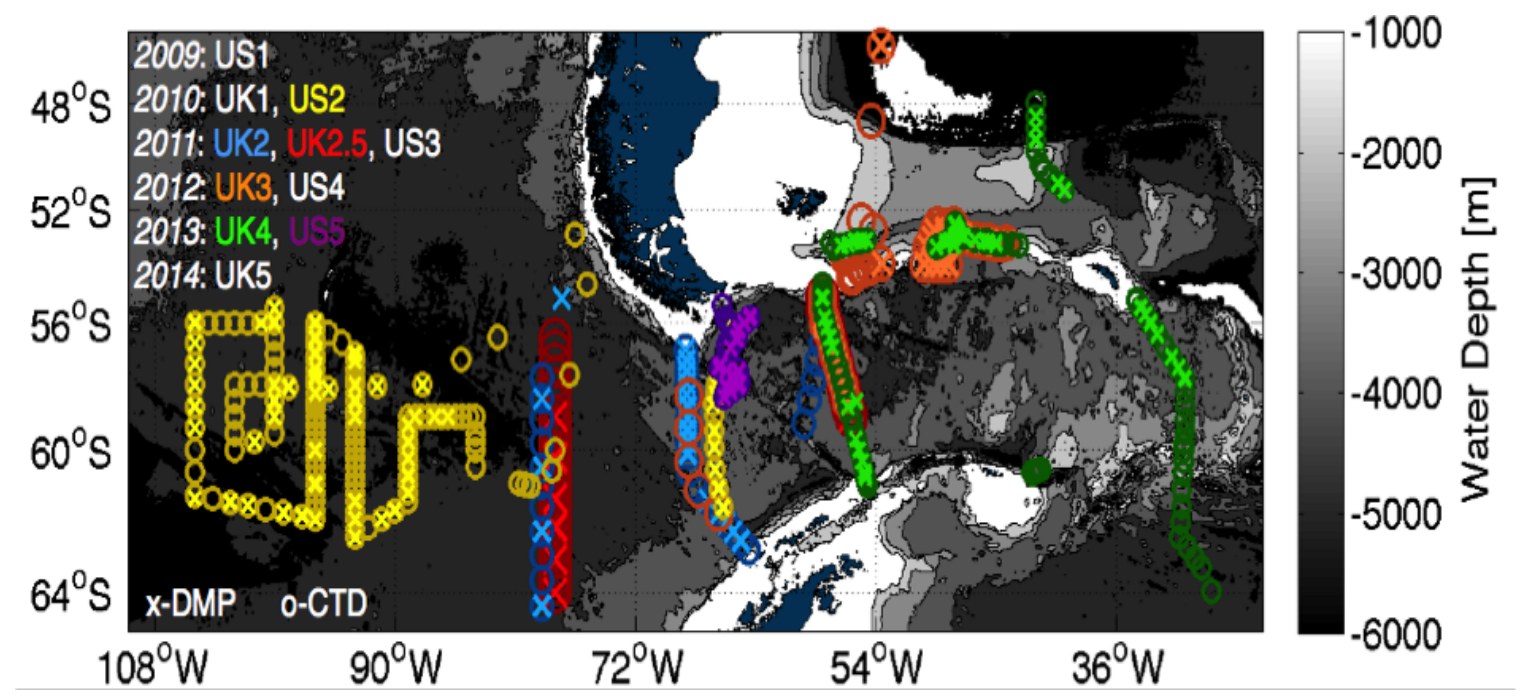

Figure 1-9: DIMES sampling, 2009-2014. Cruises which included microstructure measurements are shown in colors, deep microstructure profiles (DMP, x's) and CTD stations (o's). The water depth is shown in gray scale Smith and Sandwell (1997). The experiment sampled heavily in Drake Passage, with average winds exceeding 20 knots on most expeditions. Expeditions listed in white are not shown. 
Table 1.1: DIMES cruises with microstructure measurements

\begin{tabular}{|c|c|c|c|c|}
\hline Cruise & Ship & Dates & \# of DMP & \# of LADCP \\
\hline US2 & R/V Thomas G. Thompson & Jan-Mar 2010 & 65 & 139 \\
\hline UK2 & R/V James Clark Ross & Dec 2010-Jan 2011 & 30 & 55 \\
\hline UK2.5 & R/V James Clark Ross & April 2011 & 13 & 55 \\
\hline UK3 & R/V James Cook & Dec 2011- Jan 2012 & 47 & 100 \\
\hline UK4 & R/V James Clark Ross & Mar-Apr 2013 & 47 & 128 \\
\hline US5 & R/V Nathaniel B. Palmer & Oct-Nov 2013 & 23 & 47 \\
\hline
\end{tabular}

This thesis uses data primarily from two field expeditions of the DIMES study, hereafter referred to as the US2 and US5 expeditions (Fig 1-10 and 1-12). Both expeditions originated and ended in Punta Arenas, Chile. Each cruise included fineand microstructure profiling, CTD hydrographic measurements, and hull-mounted and lowered Acoustic Doppler Current Profiling (SADCP, LADCP) of the oceanic velocity structure.

\subsubsection{US2}

The US2 expedition, on the $\mathrm{R} / \mathrm{V}$ Thomas G. Thompson during January through March, 2010, was the most expansive of the DIMES surveys, occupying a survey grid over a nearly $10^{6} \mathrm{~km}^{2}$ region of the eastern Pacific sector of the Southern Ocean, as well as a zonal section across the Phoenix Ridge of Drake Passage. A description of the US2 study, as well as a discussion of the US2 data, has been previously presented by Ledwell et al. (2010) and St. Laurent et al. (2012).

A total of 139 measurements were taken during the US2 field programs (Fig. 110). Velocity measurements from two instruments, a $300 \mathrm{kHz}$ LADCP and a Modular Acoustic Velocity Sensor (MAVS) which is part of the High Resolution Profiler 2 (HRP2) package, were used in this study. The LADCP was mounted on the CTD rosette package which was lowered using a cable winch system. A subset of the profiles were co-located and concurrent to microstructure measurements from the free-falling profiler.

Measurements of velocity microstructure (reported here in terms of the dissipation rate of turbulent kinetic energy) were acquired at a total of 65 discrete stations: 15 stations in Drake Passage along the Phoenix Ridge and 50 stations in the upstream region of the eastern Pacific, as shown in Fig. 1-10. During the US2 survey, two profiling instrument systems were used: the HRP2, built at Woods Hole Oceano- 


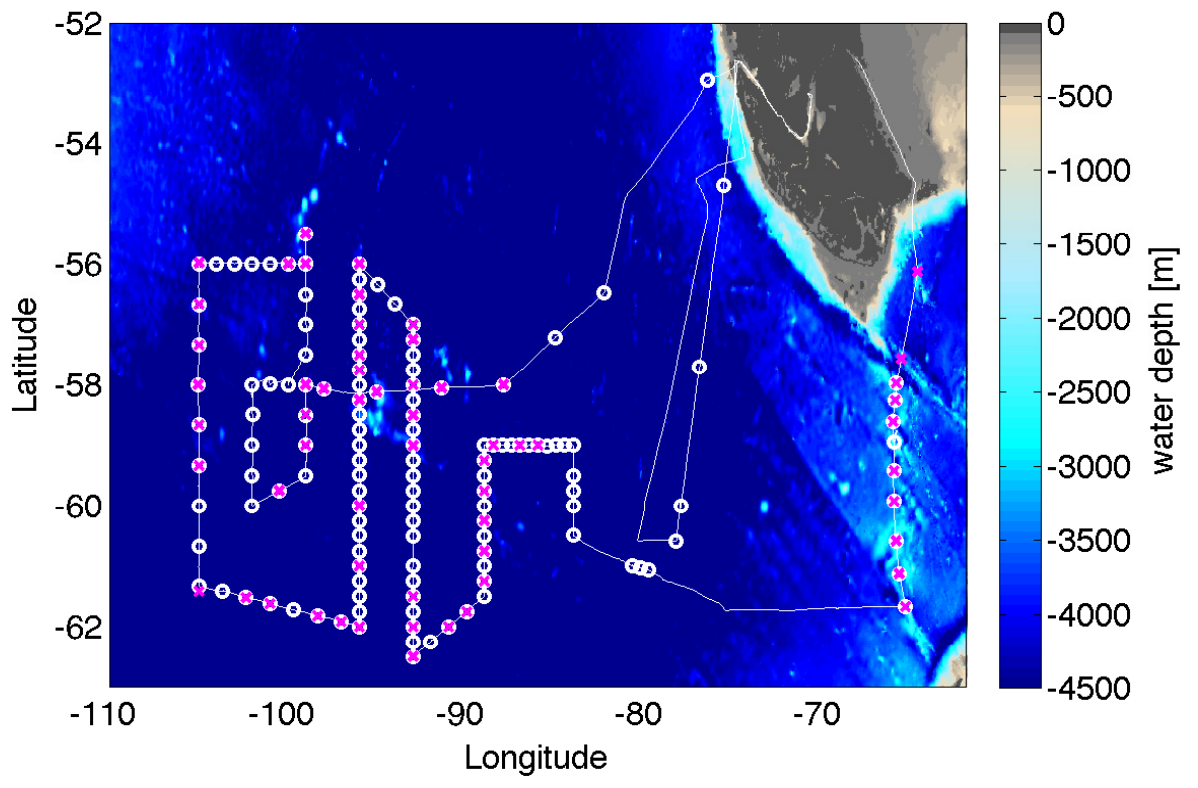

Figure 1-10: Map showing the microstructure stations (magenta $x$ ) and LADCP (white $o$ ) taken during the US2 cruise. The colors are water depth from Smith and Sandwell (1997).

graphic Institution, and the Rockland VMP-6000 (Vertical Microstructure Profiler, VMP http://www.rocklandscientific.com, Fig. 1-11), manufactured by Rockland Scientific International, Inc. HRP2 is similar to the original HRP (Schmitt et al. (1988)). Both instruments measure centimeter-scale shear and temperature from which the dissipation rates of turbulent kinetic energy, $\epsilon$, and the thermal variance, $\chi$, can be estimated. Additionally, the profilers have CTD's for measurements of temperature and salinity.

The VMP uses dual shear and thermistor probes, while the HRP2 uses two shear probes, one thermistor and one dual-needle micro-conductivity sensor. The dissipation rates reported are typically the mean of the independently estimated dissipation rates from each probe.

\subsubsection{US5}

The US5 expedition, on the RVIB Nathaniel B. Palmer, between October and November, 2013, was the first DIMES measurement program done during Austral Spring. The survey grid was situated over the Subantarctic Front and Polar Front regions, 


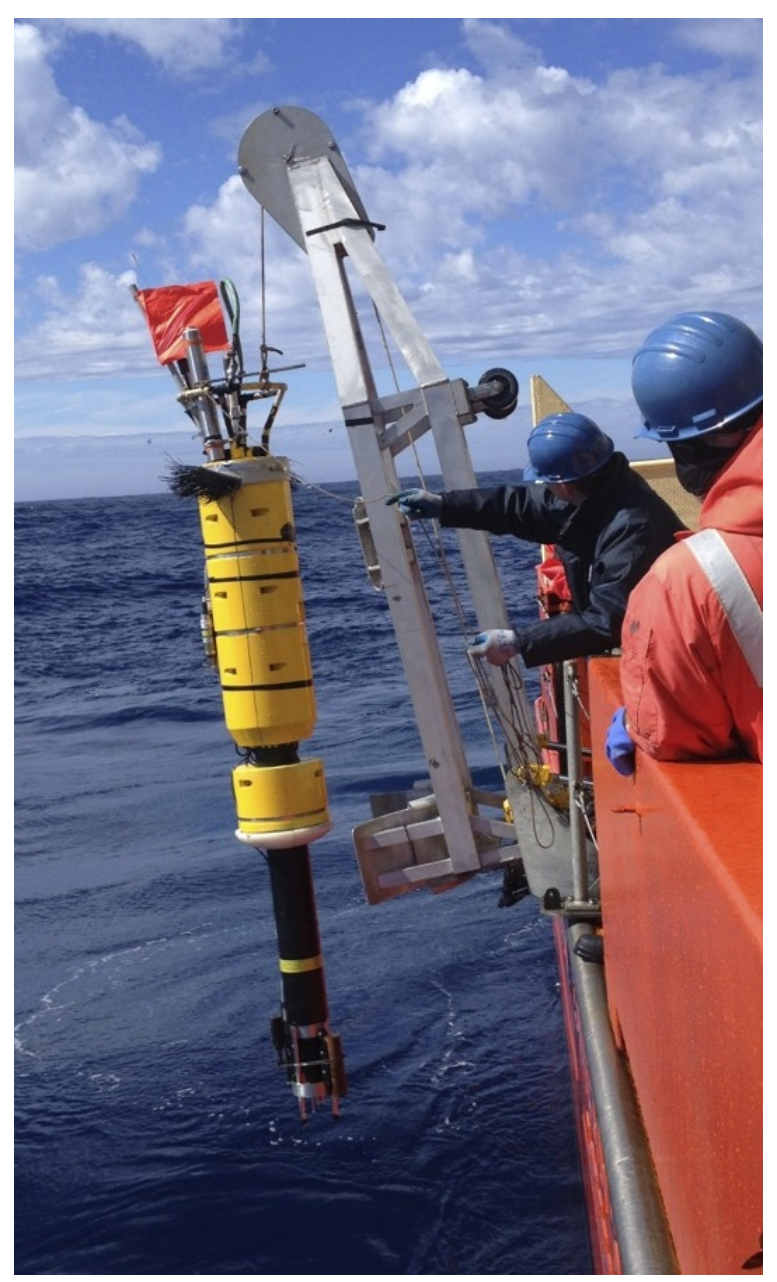

Figure 1-11: A Rockland Scientific deep microstructure profiler is deployed off the stern of the Nathaniel B. Palmer during a cruise in Drake Passage.

downstream of the Phoenix Ridge and extending over the seafloor topography of the Shackleton Fracture Zone. US2 and US5 are the only DIMES expeditions to survey the Drake Passage region, which we define as the region between Cape Horn and the Antarctic Peninsula. In this region the various fronts of the Antarctic Circumpolar Current (ACC) are often compressed to the northern half of the passage, enhancing the flow over the highly variable seafloor topography of the region.

The measurements of velocity and finestructure and microstructure were made using the same instruments and techniques as on US2. US5 included 47 lowered $300 \mathrm{kHz}$ ADCP profiles and 23 microstructure profiles. During the US5 survey, only the RVMP was operated for deep profiling. A similar profiling instrument, a VMP-250, was also used in the collection of these data. The sensor suite and analysis is the same, but the instrument is tethered to the ship and deployed using a winch system. 


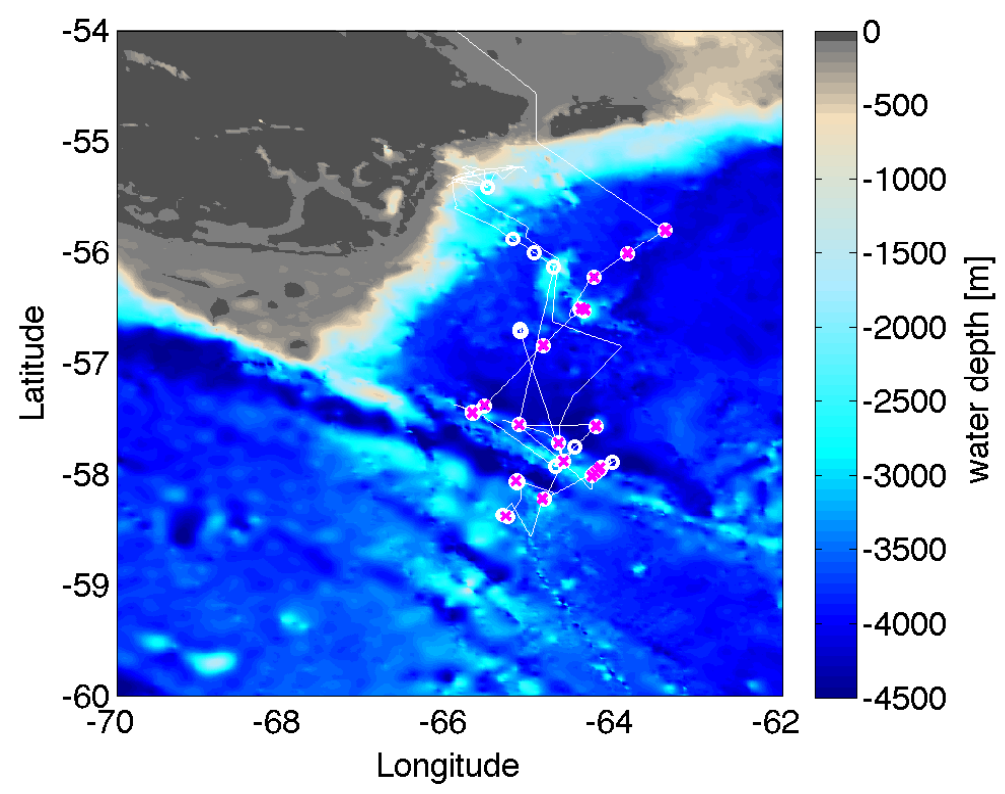

Figure 1-12: Same as Fig: 1-10 but for US5.

US5 had the most comprehensive set of meteorological measurements of all the DIMES cruises. Meteorological sensors were mounted on a science mast $15 \mathrm{~m}$ above water level typically at the center of the ships used in the DIMES field programs. Measurements of wind speed and direction, pressure, long-wave and short-wave radiation, humidity, and air temperature were taken at 1 second sampling intervals. A thermosalinigraph provided measurements of sea surface temperature. Bulk fluxes are estimated using the COARE Bulk flux algorithm (version 2, Fairall et al. (1996)). Measurements were averaged to 15 min prior to bulk flux computation, and averaged to 1 hour afterwards (Fig. 1-13). During the US2 cruise, the air temperature sensor failed prohibiting the calculation of the turbulent heat fluxes.

Eight surface drifters from the Global Drifter Program (GDP) were deployed in pairs in the Polar Front region during US5 (Fig. 1-14). Each drifter consisted of a surface buoy tethered to a drogue at $15 \mathrm{~m}$. The drifters use Global Positioning System (GPS) technology and Iridium capabilities to report their locations. This incurs a minimal position error which is estimated to be on the order of 10 meters. The zonal and meridional velocities are computed using the basic equations, $u=\frac{d x}{d t}$ and $v=\frac{d y}{d t}$ where the time step in communication, $d t$ is 1 hour. The resulting velocity estimates are considered to be representative of the surface velocity. More details on these drifters are given in (Centurioni (2011)). 

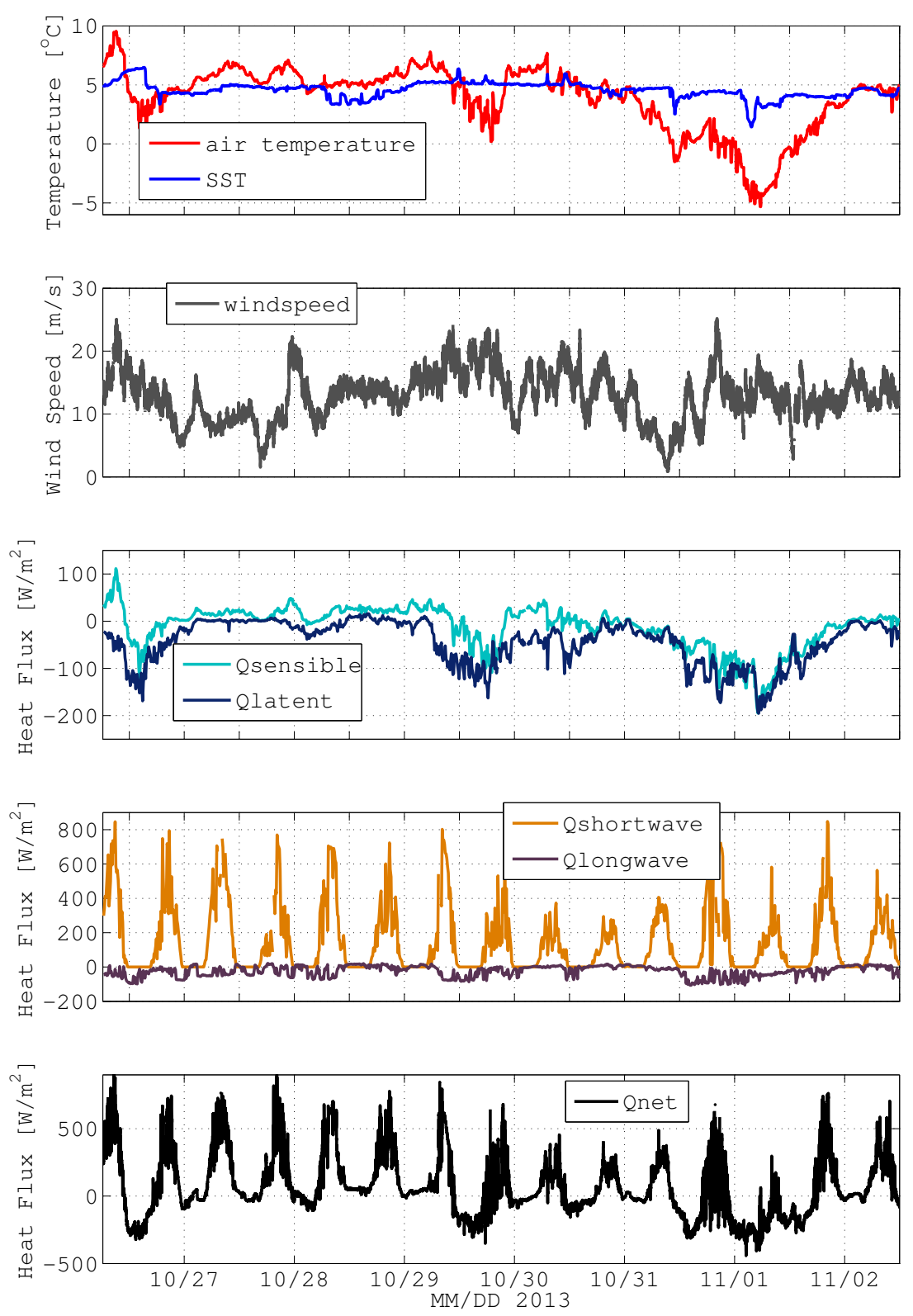

Figure 1-13: Air temperature, sea surface temperature, windspeed, sensible, latent, shortwave, longwave and net heat fluxes during the US5 field expedition. The ship occupied a localized sampling area encountering storm activity multiple times. 

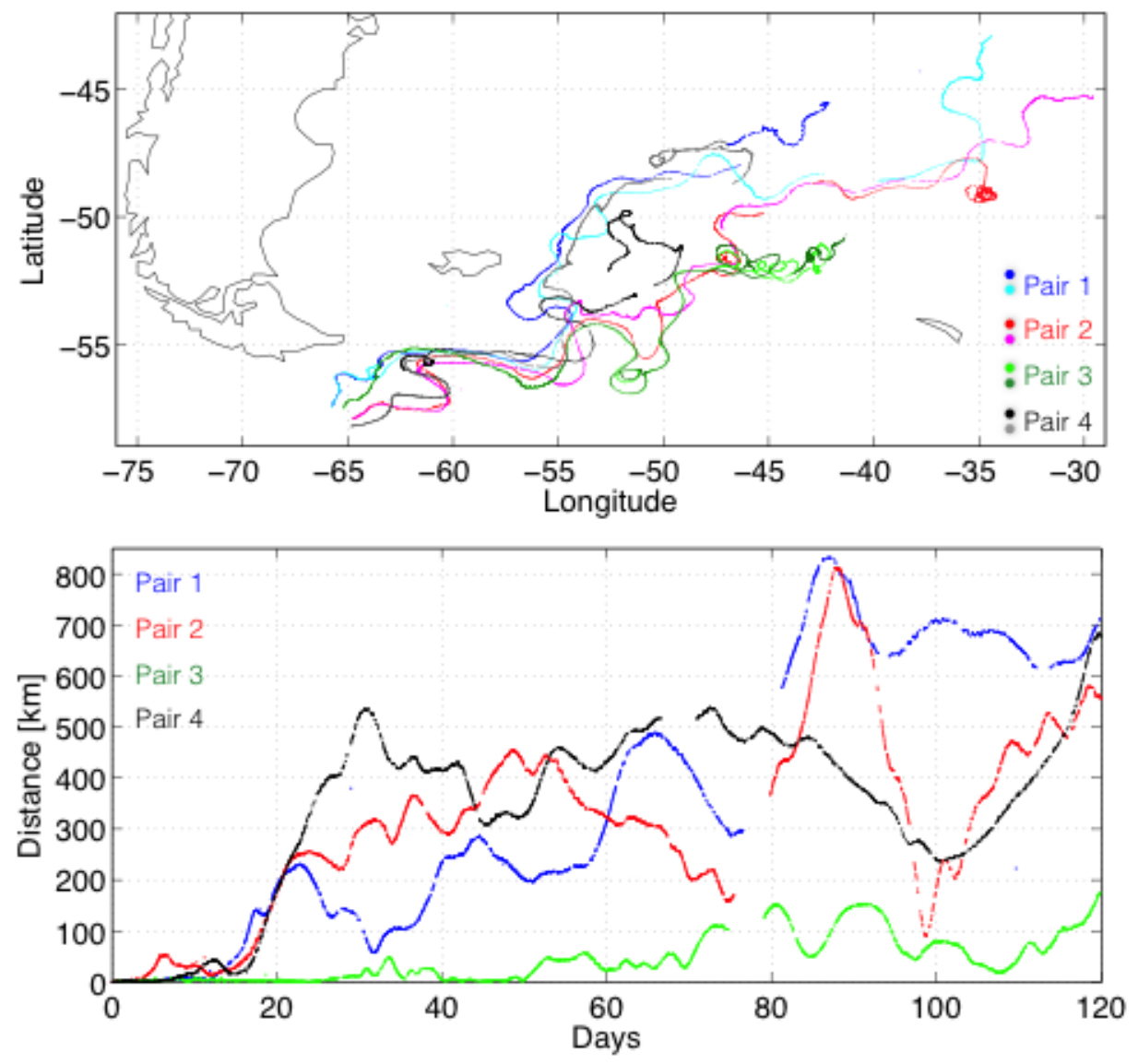

Figure 1-14: Trajectories of 4 pairs of drifters deployed during the DIMES US5 cruise (Oct 2013). The distance between each pair is shown in the lower panel show for a 4 month period. The drifters are part of the Surface Velocity Program (SVP) with a $15 \mathrm{~m}$ drogue. 


\subsection{Outline}

This thesis uses data from DIMES to study upper ocean processes that lead to turbulence. Chapter 2 addresses the discrepancy between finestructure parameterizations and observed microstructure based rates of mixing in intrusive regions of the water column. Enhanced thermohaline variability in frontal regions can bias indirect methods that relate internal wave shear and strain to dissipation.

Chapter 3 explores the mixing implications of strong intrusions in the Southern Ocean. Intrusions in temperature and salinity are common in the upper $1000 \mathrm{~m}$, with temperature changing by as much as $2^{\circ} \mathrm{C}$ over $50 \mathrm{~m}$ vertical scales. These large gradients in temperature and salinity are density compensated, but support double diffusive instability. This instability is due to the difference in the molecular diffusivity of heat and salt. In Chapter 4, I show that this form of instability can develop in the Southern Ocean contributing to enhanced values of mixing. Mixing rates can be 3 times larger than estimates that only consider internal wave breaking.

Chapter 4 studies the near-surface layer focusing on the structure of turbulent dissipation and shear in the mixed layer. Conventional scalings are unable to account for the observed structures likely due to the extreme wind and surface wave state. Breaking surface waves and Langmuir turbulence are considered as mechanisms causing the structure of turbulent dissipation to vary from classical scalings.

Chapter 5 addresses the fate of wind-generated energy in the wake of atmospheric storms. Strong surface fluxes of momentum during the passage of storms excites mixed-layer inertial currents, which can feed energy into the internal wave field below the ocean layer that directly interacts with the atmosphere. We estimate the nearinertial energy flux from the wind to mixed layer motions using in-situ data collected in Drake Passage. We consider finestructure internal wave energy and dissipation at mid-depth, finding the weakest values upstream of Drake Passage.

The research presented in this dissertation involves analysis of data collected from turbulence microstructure profilers, surface drifters, meteorological sensors, CTD, and acoustic Doppler current profilers in one of the most energetic and remote locations in the world ocean. These data are used to study processes that support mixing, and to quantify the rates of mixing measured via direct measurements. The resulting conceptual framework will help to ground truth parameterizations used in global map 
estimates and to provide insight into dominant processes in the upper ocean in Drake Passage. 


\section{Chapter 2}

\section{Biases between finestructure and microstructure based mixing estimates}

\subsection{Introduction}

The global distribution of diapycnal mixing has significant impact on the large-scale ocean circulation (Wunsch and Ferrari (2004)). Microstructure measurements provide the most accurate estimates of turbulent dissipation, but they require specialized instrumentation (e.g Lueck (2002)) and are scarce in the global ocean. To study mixing rates on regional and global scales, many studies have implemented finescale parameterizations based on estimates of internal wave shear and strain (e.g. Naveira Garabato et al. (2004), Kunze et al. (2006), Wu et al. (2011), Whalen et al. (2012)). These parameterizations are based on the assumption that turbulent dissipation results from a continuum of internal wave-wave interactions that transfer energy downscale.

Climate models do not resolve the physical processes that contribute to diapycnal mixing, hence, assessing the accuracy of current mixing parameterizations and developing new parameterizations for diapycnal mixing are active areas of research (e.g. Polzin et al. (2014), Thurnherr et al. (2015)). To obtain realistic parameterizations of the dissipation rate of turbulent kinetic energy, $\epsilon$, interpreting mixing observations in the context of the underlying dynamical processes is essential. Here, we use the 
DIMES data set to assess scalings developed for internal wave-driven mixing which has been hypothesized to be a dominant mixing mechanism in the stratified ocean interior.

Prior to DIMES, the Southern Ocean (SO) was believed to be a region of enhanced mixing due to strong atmospheric forcing, steep topography, and the Antarctic Circumpolar Current (ACC), yet few direct measurements of turbulence existed in the SO to validate this assumption. Instead, most estimates of mixing in the SO prior to DIMES were based on parameterizations applied to finestructure velocity and hydrographic data. In this chapter, we review notable previous estimates of mixing that helped motivate the DIMES study and compare our microstructure observations obtained during two cruises in the Drake Passage with these estimates. We find that previous dissipation rates and diffusivity estimates were biased high relative to mixing rates based on DIMES microstructure measurements, often by an order of magnitude.

The study is organized as follows. In Section 2.1.1, we show that dissipation rate and diffusivity estimates in the Drake Passage estimated from LADCP observations (Naveira Garabato et al. (2004)) are larger than those estimated with shear microstructure measurements (St. Laurent et al. (2012)). In Section 2.1.2, we review estimates of turbulent dissipation and diffusivity based on finestructure hydrography following Thorpe (1977), and Kunze et al. (2006). The observations used in this analysis are described briefly in Section 2.2. Section 2.3 provides a detailed comparison of the observed microstructure estimates and a strain-based parameterization of dissipation rates (Whalen et al. (2012)). We conclude with a discussion of the differences between the microstructure and strain-based estimates in section 2.4.

\subsubsection{Estimates based on finestructure shear}

Mixing parameterizations based on finescale shear are among the most well-developed formulations for estimating turbulence levels in the ocean interior. These parameterizations are based on the pioneering work of Gregg (1989) with important refinements provided by Polzin et al. (1995), Gregg et al. (2003) and others. Empirical relationships were determined using shear measured at the 10-m scale by specialized profiling systems (Sanford et al. (1982), Schmitt et al. (1988), Lueck (2002)).

With the proliferation of acoustic Doppler current measurements, considerable at- 
tention has been focused on using vertical shear estimates from Doppler profilers to estimate dissipation rates. Conventional acoustic Doppler current systems resolve velocity variations to vertical scales of generally between 50-100 m (Polzin et al. (1995), Thurnherr et al. (2015)). To address resolution limitations of shear measurements, Polzin et al. (2002) proposed an empirically derived transfer function that accounts for the loss of measured velocity variance at smaller vertical scales.

The Polzin et al. (2002) adaptation of shear based mixing parameterization may lead to differing estimates of diffusivity depending upon the values of a shear to strain ratio, $R_{w}$. The parameterized diffusivity is proportional to $R_{w}=\frac{\left\langle V_{z}^{2}>\right.}{N^{2}<\zeta_{z}^{2}>}$ (Kunze et al. (1990), Polzin et al. (1995)), where $\left\langle V_{z}^{2}\right\rangle$ is the internal wave shear, $<\zeta_{z}^{2}>$ the internal wave strain, and $\bar{N}^{2}$ is a mean buoyancy frequency. Here, the overbar denotes the buoyancy frequency calculated over a large vertical scale, and the brackets denote ensemble averages. $R_{w}$ can be thought of as an aspect ratio of the horizontal kinetic energy to the potential energy and details the frequency content of the wave field. When both velocity shear and density measurements are available, $R_{w}$ may be estimated directly. One of the first applications of the Polzin et al. (2002) adaptation was by Naveira Garabato et al. (2004), who used lowered Acoustic Doppler Current Profiler (LADCP) measurements from a collection of large-scale hydrographic surveys taken in the Drake Passage through the Scotia Sea. They inferred large diffusivity levels, from $\mathcal{O}\left(10^{-4}\right) \mathrm{m}^{2} \mathrm{~s}^{-1}$ to $\mathcal{O}\left(10^{-3}\right) \mathrm{m}^{2} \mathrm{~s}^{-1}$, below approximately 1000$m$ depth throughout the Drake Passage. In contrast, the same parameterization used by Naveira Garabato et al. (2004) has been used in the global ocean by Kunze et al. (2006) who inferred more modest values of deep diffusivity, from $\mathcal{O}\left(10^{-5}\right) \mathrm{m}^{2} \mathrm{~s}^{-1}$ to $\mathcal{O}\left(10^{-4}\right) \mathrm{m}^{2} \mathrm{~s}^{-1}$, with local enhancements generally confined to depths below $1500 \mathrm{~m}$. Notably, Kunze et al. (2006) included data used by Naveira Garabato et al. (2004) highlighting the discrepancies in the implementation of the parameterization. The differences in diffusivities estimated in the aforementioned studies are in part due to the variability in values of $R_{w}$.

Recent studies have shown discrepancies between dissipation and diffusivity estimates from microstructure measurements and finestructure parameterizations (Sheen et al. (2014), Waterman et al. (2014)) in the Southern Ocean. Both Sheen et al. (2014) and Waterman et al. (2014) showed that the shear based parameterization overestimates observed mixing rates in near-bottom regions of strong internal wave generation. The parameterization relies on the assumption that the background internal-wave field is well characterized by the Garrett-Munk (GM) spectrum. The frontal region of the 

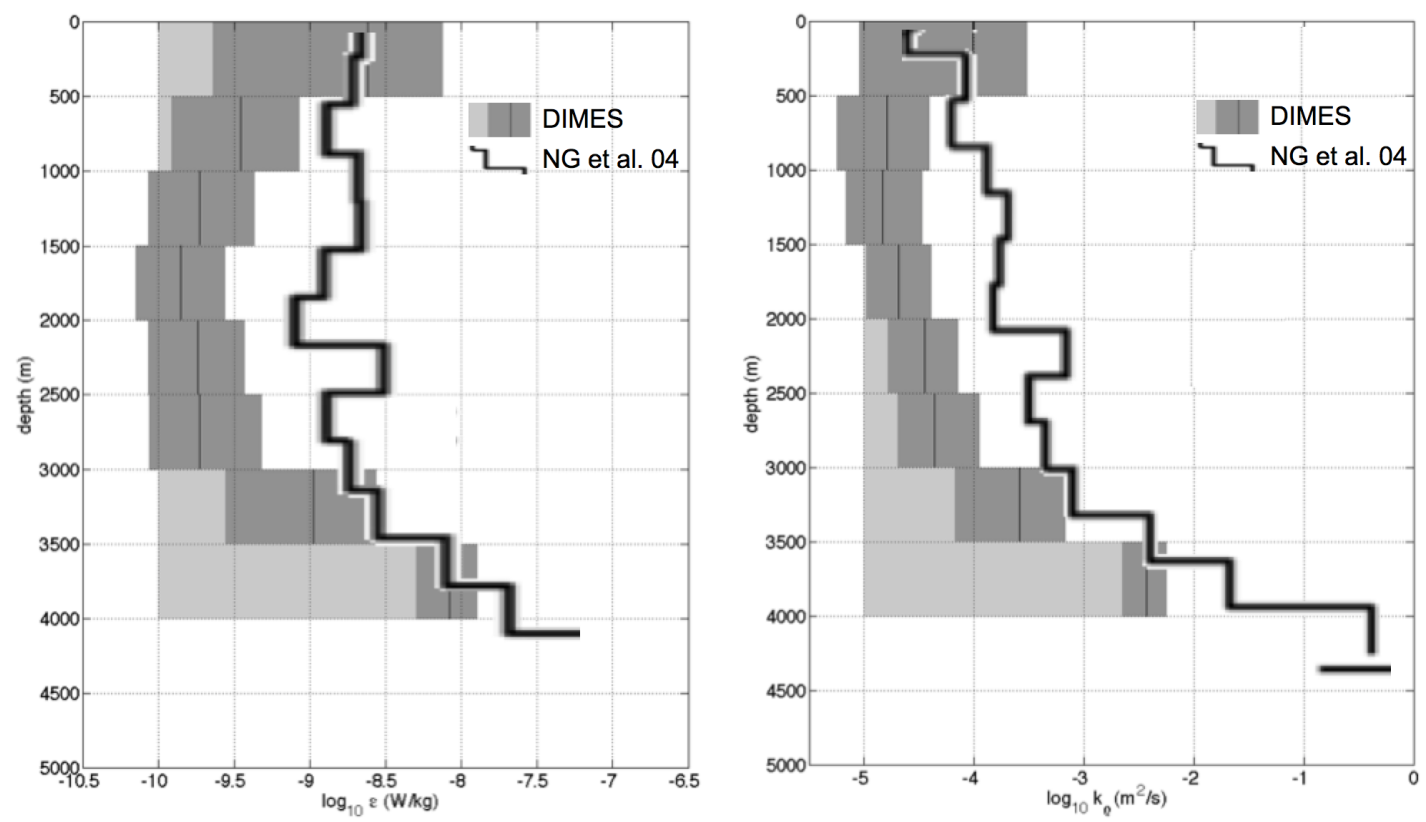

Figure 2-1: Comparison of dissipation rate and diffusivity estimates from two studies that examined turbulence and mixing levels along the 'Albatross' section crossing Drake Passage. The estimates of Naveira Garabato et al. (2004) used LADCP data and the Polzin et al. (2002) shear-based parameterization. The DIMES estimates were based on shear microstructure collected in 2010 (St. Laurent et al. (2012)).

Southern Ocean fails this characterization because it is forced by energetic internal waves that originate as lee waves in the deep flow over topography. As discussed by Waterman et al. (2014), the extent to which the internal wave environment of the Southern Ocean can be modeled by the GM spectrum is unclear.

A comparison of diffusivity estimates in the Drake Passage region obtained from microstructure measurements (St. Laurent et al. (2012)) and finestructure parameterizations (Naveira Garabato et al. (2004)) shows discrepancies up to an order of magnitude in the Drake Passage region (Fig. 2-1). The discrepancy is the most pronounced at mid-depth (between 500-200m). Naveira Garabato et al. (2004)'s anomalously large dissipation estimates based on velocity shear are the result of two contributing issues. First, noise levels in the ADCP-derived shear spectrum become acute at wavelengths shorter than $50 \mathrm{~m}$. In the case of Naveira Garabato et al. (2004), the inclusion of noisy shear variance at short wavelengths biased their estimates high (Kunze et al. (2006)). Second, Naveira Garabato et al. (2004) estimated values of $R_{w}$ between 8 and 12, using a bulk value of $R_{w}=10$ in their study. This value is significantly higher than the GM value of 3, which suggests that there is enhanced near-inertial 
internal wave energy relative to the GM spectrum in the Southern Ocean. Kunze et al. (2006) estimated a globally averaged value of $R_{w}=7$, while Waterman et al. (2013) found that $R_{w}$ was much lower than 7 in the SO in regions of rough topography and strong frontal flows. While the values of Naveira Garabato et al. (2004) are recognized to be biased high, discrepancies between finescale parameterizations and microstructure based mixing rates require further study, especially at mid-depth in the Drake Passage region.

\subsubsection{Estimates based on finestructure hydrography}

Thorpe (1977) proposed that density overturns in hydrographic profiles may be related to mixing parameters. The Thorpe scale method is attractive because of the difficulties and costs associated with measuring microscale quantities, and because of the wide availability of hydrographic profiles from a variety of shipboard and autonomous platforms including Argo floats. Parameterizing turbulent dissipation from density overturns requires estimates of the Thorpe scale, $L_{t}$, which is proportional to the distance a water parcel must move to obtain a statically stable density profile. $L_{t}$ is related to the Ozmidov scale, $L_{o}$, the scale at which turbulent eddies are affected by buoyancy. The turbulent dissipation $\epsilon$ is related to $L_{o}$ through the relationship:

$$
\epsilon=c L_{o}^{2} N^{3}
$$

where $c$ is a constant often taken to be 0.8 (Dillon (1982)).

In the Southern Ocean, the Thorpe scale method has been used on hydrographic data from Argo floats, shipboard CTDs, and expendable CTD (XCTDs). In particular, Thompson et al. (2007) applied a Thorpe scale analysis to XCTD data from Drake Passage (along the World Ocean Circulation Experiment (WOCE) transect AX22) and obtained diffusivities in the range of $\mathcal{O}\left(10^{-3}\right) \mathrm{m}^{2} \mathrm{~s}^{-1}$ to $\mathcal{O}\left(10^{-2}\right) \mathrm{m}^{2} s^{-1}$ in the upper 1000m (Fig. 2-2). Frants et al. (2013) found that the Thompson et al. (2007) diffusivities were biased high by 1-2 orders of magnitude for reasons related to the detection of overturns with poor signal to noise resolution. Mixing rates derived from the DIMES US2 data (described in Section 1.5.1) show discrepancies with Thompson et al. (2007)'s Thorpe scale estimates (Fig. 2-2). These discrepancies are consistent with the results of Frants et al. (2013). 

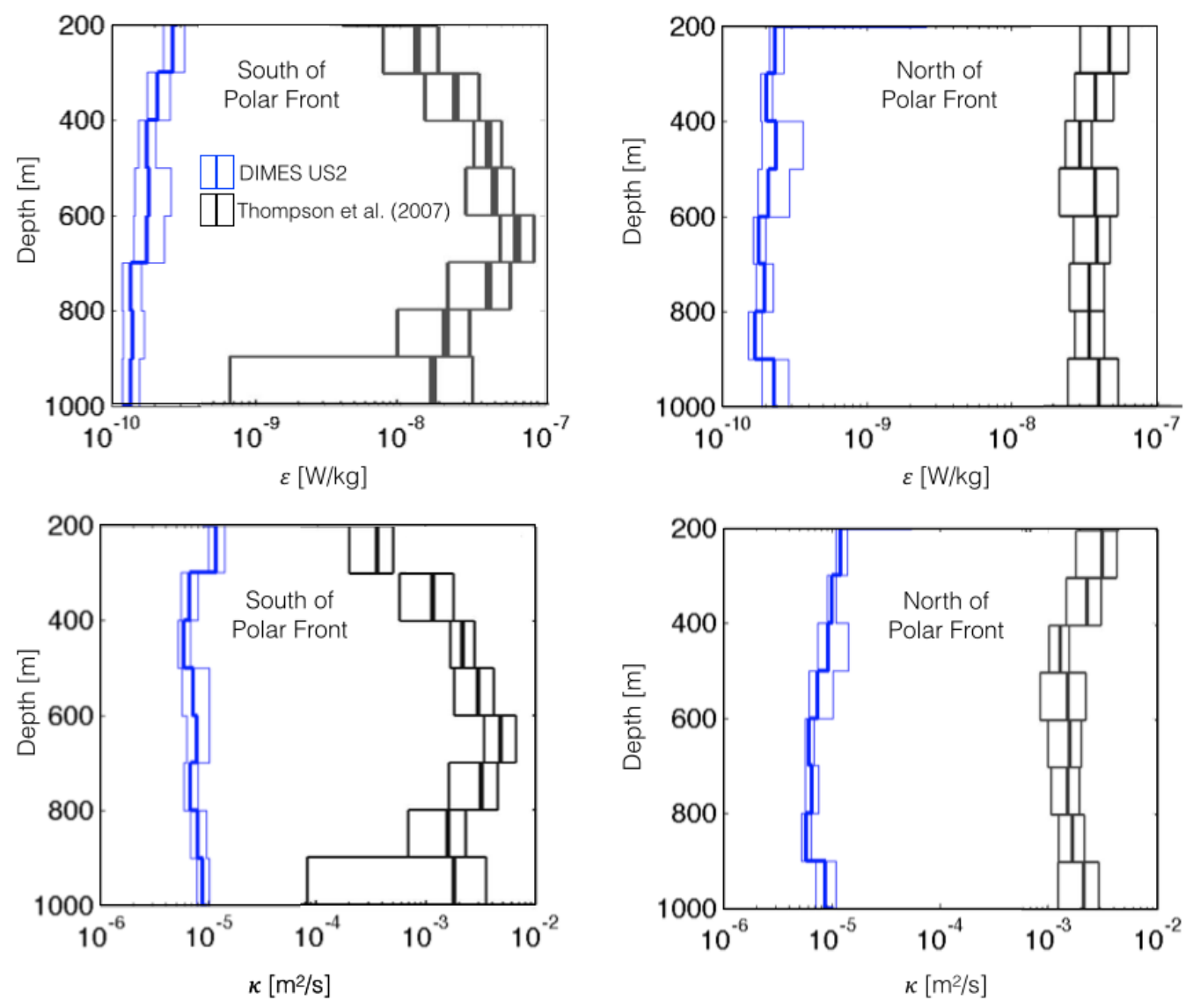

Figure 2-2: Comparison of turbulent dissipation and and diffusivity estimated by Thompson et al. (2007) (black) to estimates from DIMES (blue) in the same region.

Finescale strain, defined as the vertical derivative of isopycnal displacements, may also be used to estimate dissipation from hydrographic data. Wijesekera et al. (1993) adapted the early methods of Gregg (1989) to relate the strain variance to the turbulent energy dissipation rate. Kunze et al. (2006) applied this method to World Ocean Circulation Experiment (WOCE) LADCP measurements. This method also relies on the shear-to-strain ratio, which in the absence of shear measurements is often assumed to be $R_{w}=3$ based on Garrett and Munk (1972). Other studies based on Argo float data in the Southern Ocean (Wu et al. (2011)) and globally (Whalen et al. (2012)) use $R_{w}=7$ and $R_{w}=3$, respectively, in their estimates of dissipation. The strainbased dissipation estimates of Kunze et al. (2006) and Whalen et al. (2012) have been converted to diffusivity maps using the Osborn (1980) relation $\left(\kappa_{\rho}=\Gamma \frac{<\epsilon>}{\left\langle N^{2}\right\rangle}\right)$ yielding a range of diffusivity values between $\mathcal{O}\left(10^{-5}\right) m^{2} s^{-1}$ to $\mathcal{O}\left(10^{-3}\right) m^{2} s^{-1}$ in the Drake Passage region. 


\subsection{Data}

The data used in this study were collected during DIMES cruise US5 in northern Drake Passage, in both the Polar and Subantarctic Fronts. CTD and LADCP profiles were collected at 47 stations, and 23 full depth microstructure stations were occupied with the VMP. Further details on the dataset are available in Chapters 1 and 3 and in Merrifield et al. (2016). Three stations were occupied for 14, 17, and 14 hours respectively for time series sampling and are shown in 2-3 in white boxes.

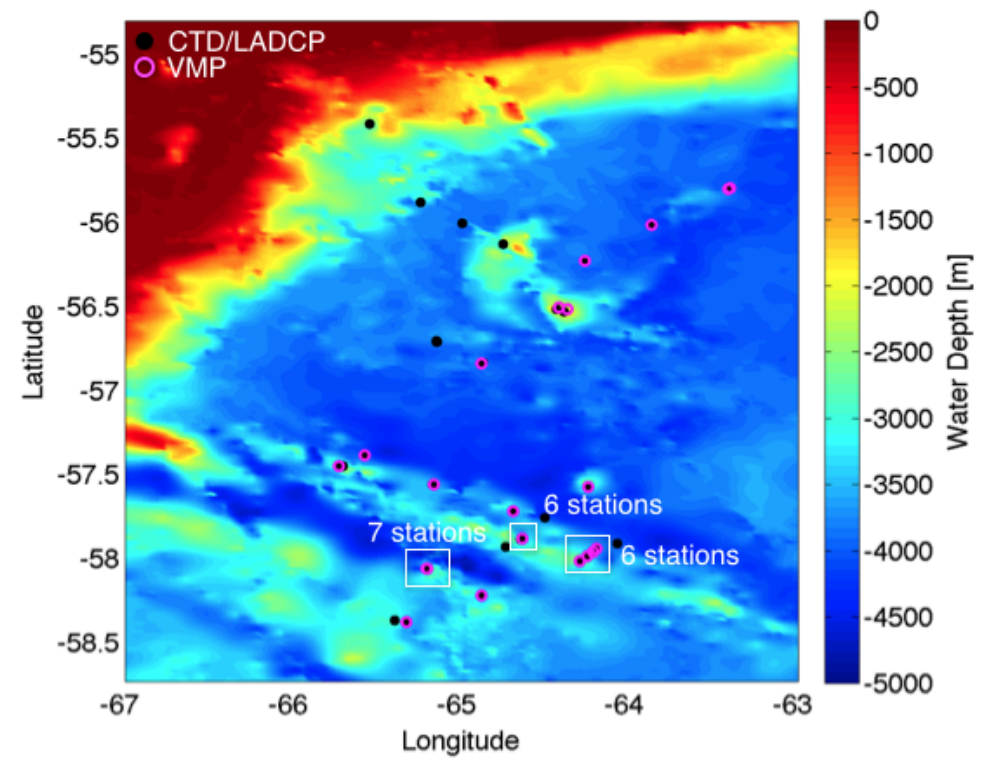

Figure 2-3: Map of CTD/LADCP and VMP stations taken during US5 overlaid on water depth from Smith and Sandwell (1997). The location of the time series stations and the number of casts delineated by the white boxes.

\subsection{Results}

Estimates of velocity shear require specialized instruments and are scarce in the global ocean relative to hydrographic measurements. With the advent of global observing networks such as Argo, global coverage of hydrographic measurements has increased significantly. The strain parameterization described in Section 2.2.1 has become popular because global maps of diffusivity may be generated from hydrographic measurements. Here, we compare our estimates of turbulent dissipation obtained from 
Vertical Microstucture Profiler (VMP) measurements during DIMES cruise US5 (OctNov 2013) within the Drake Passage region of the Southern Ocean with those from the strain-based parameterization of Whalen et al. (2012). We apply the method used to estimate strain and assess the physical assumptions of the parameterization. The estimates are examined in three distinct depth regimes in the sampling region; the near surface layer, the interleaving layer, and the stratified interior. In Austral Spring in northern Drake Passage, mixed layers are shallow $(<200 \mathrm{~m})$ and comprised of the lightest waters in the region with potential densities less that $1027.1 \mathrm{~kg} / \mathrm{m}^{3}$ (Fig. 2-4). At intermediate depths, between $200-1000 \mathrm{~m}, \mathrm{~T} / \mathrm{S}$ variance is enhanced in potential density classes between $1027.1-1027.5 \mathrm{~kg} / \mathrm{m}^{3}$ due to interleaving water masses (Merrifield et al. (2016); chapter 3). Below that, the stratified interior is salinity stratified at depths shallower than $1800 \mathrm{~m}$ and temperature stratified at deeper depths.

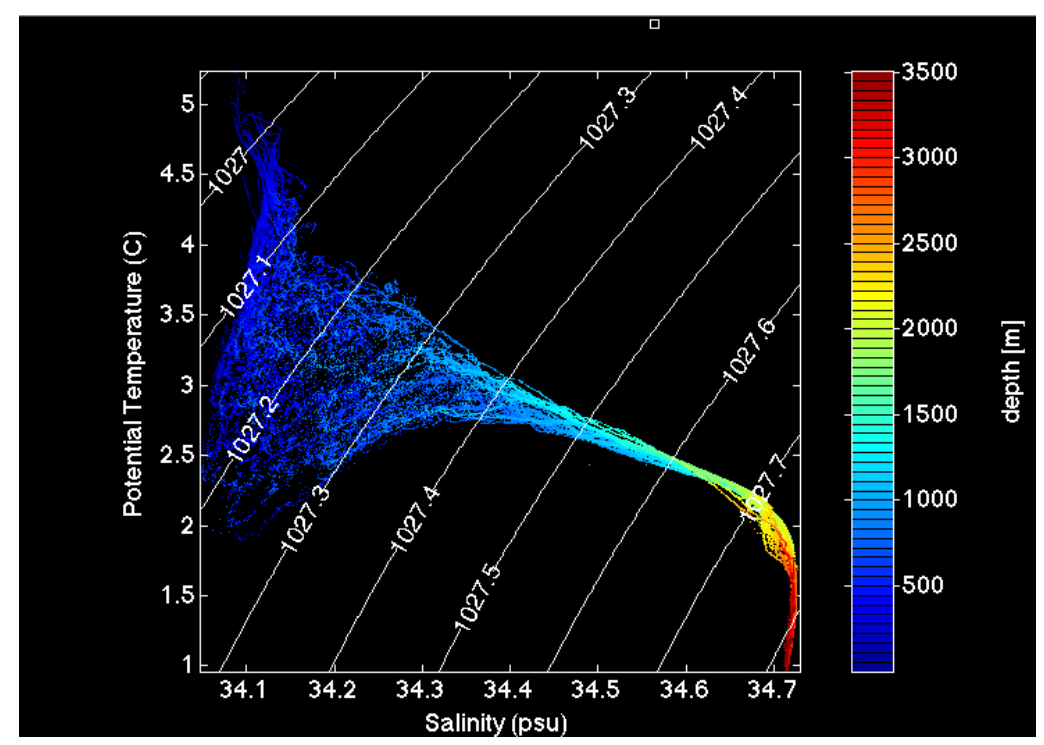

Figure 2-4: Potential temperature versus salinity for measurements made during the US5 expedition. The black line contours overlaid are potential density in units of $k g / m^{3}$.

The three depth regimes exhibit distinct patterns in the turbulent dissipation rates of kinetic energy, $\epsilon$ and thermal variance, $\chi$. To characterize the variability of $\epsilon$ and $\chi$ in density space, we estimate probability density functions (PDFs) of both parameters are presented in the three depth regimes, delineated by density, and compared to the full PDFs over all densities (Fig. 2-5). For example, PDFs in the near surface layer $\left(\sigma<27.1 \mathrm{~kg} / \mathrm{m}^{3}\right.$, top panels, $8 \%$ of total distribution), where $\epsilon$ and $\chi$ are often elevated, are shown in red relative to PDFs of the entire water column which are shown in blue. In the interleaving layer $(27.1<\sigma<27.5$, middle panels, $32 \%$ of distribution), the $\epsilon$ PDF 
is very similar to that of the full dataset, whereas the $\chi \mathrm{PDF}$ is significantly enhanced. In the stratified interior $\left(\sigma>27.5 \mathrm{~kg} / \mathrm{m}^{3}\right.$, bottom panels, $60 \%$ of distribution), the PDFs of $\epsilon$ are indistinct from the full depth PDFs while the PDF of $\chi$ in this depth range has fewer high values than the full distribution.

The differences among the PDFs in the three regimes suggest that distinct dynamical processes may contribute to the observed mixing rates. In the deepest layers, bottom-generated lee-waves (see section 1.3.1) that break locally may lead to enhanced turbulence (e.g. St. Laurent et al. (2012)). In the interleaving region, the results presented in chapter 3 support the hypothesis that double-diffusive instability likely is contributing to enhanced rates of diapycnal diffusivity (see also Merrifield et al. (2016)). The different dynamical regimes are important because the finestructure parameterizations described in sections 2.1.1 and 2.1.2 are based on the assumption that mixing is driven by internal wave breaking. In particular, the finescale methods rely on the assumption that the background wave field is GM and that the shear and strain fluctuations are due to internal wave activity. The present results suggest that thermohaline variability and double-diffusive instability may affect the diffusivity estimates used in the finescale parameterizations. 


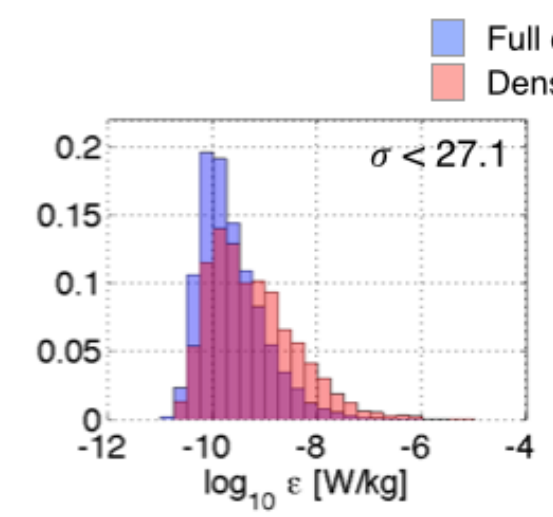

Full distribution

Density range distribution
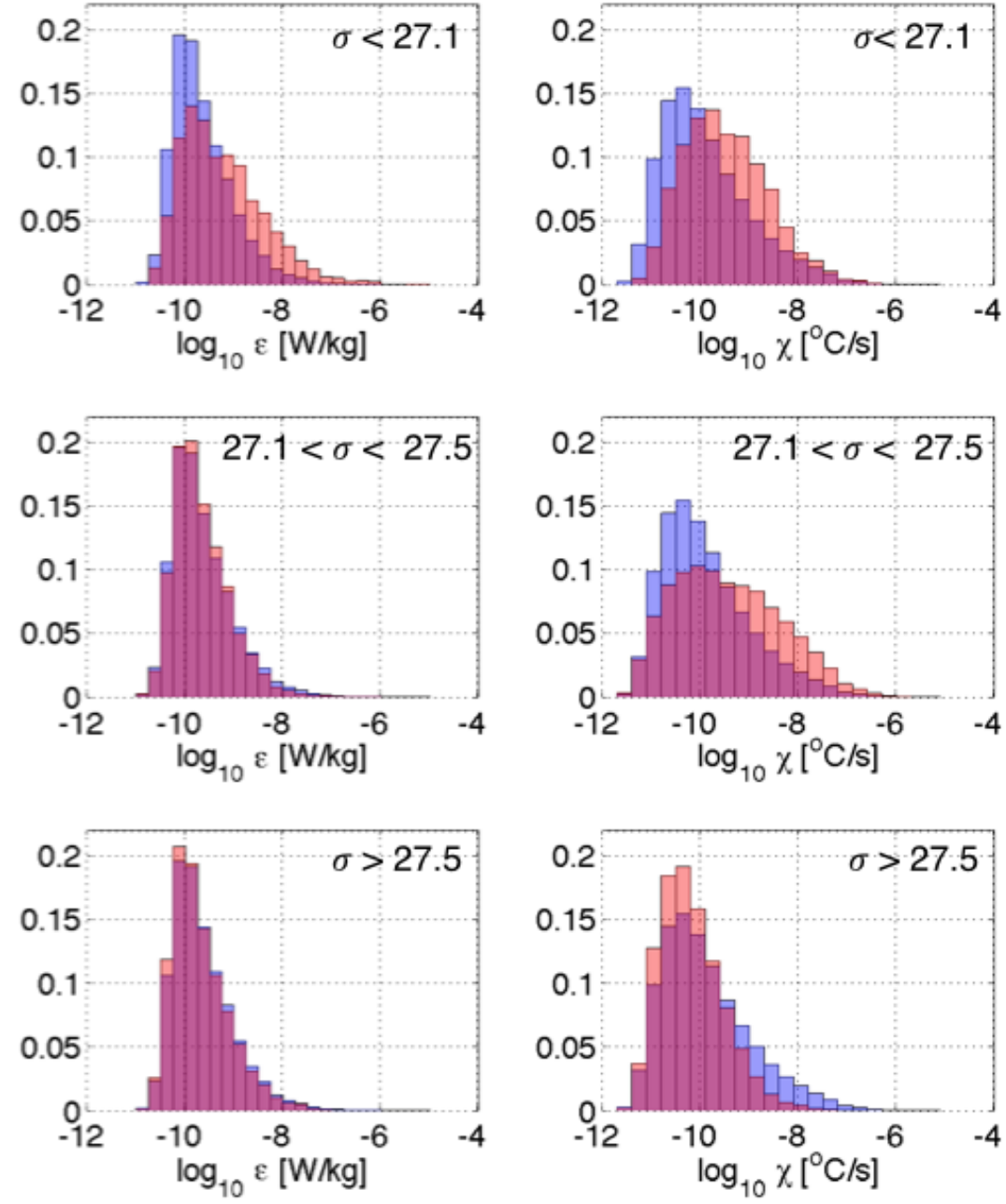

Figure 2-5: PDF's of $\epsilon$ and $\chi$ in the near-surface, interleaving, and weakly stratified depth regimes relative to the full PDF over all depths. There is a distinct elevation in $\chi$ in the interleaving region where $\mathrm{T} / \mathrm{S}$ variance is significant. The dark purple color represents an overlap in the two PDF's.

\subsubsection{Strain parameterization}

Following Whalen et al. (2012)'s study based on Argo hydrographic data, we estimate strain from the US5 CTD data as

$$
\zeta_{z}=\frac{N^{2}-N_{r e f}^{2}}{\overline{N^{2}}}
$$


where $N^{2}$ is the buoyancy frequency estimated from each potential density profile, $N_{r e f}^{2}$ is a quadratic fit of the buoyancy frequency in $200 \mathrm{~m}$ half-overlapping segments, and the over-bar represents the vertically averaged value of $N^{2}$ in each segment. Strain variance is obtained by integrating the strain wave number spectrum, $\phi$,

$$
\left\langle\zeta_{z}^{2}\right\rangle=\int_{\min \left(k_{z}\right)}^{\max \left(k_{z}\right)} \phi(m) d m
$$

The integration limits were fixed at $1 / 100 m$ and $1 / 40 m$, except in the case that $\left\langle\zeta_{z}^{2}\right\rangle>0.2$. In that case we adjust the value of $\max \left(k_{z}\right)$ was adjusted to satisfy the condition $\left\langle\zeta_{z}^{2}\right\rangle<0.2$. We also integrate the GM strain variance $\left\langle\zeta_{z G M}^{2}\right\rangle$ defined as (2.3) with $\phi_{G M}$ therein over the same wavenumber range including adjustments of $\max \left(k_{z}\right)$.

The parameterization of $\epsilon$ based on strain variance is:

$$
\epsilon_{\text {fine }}=\epsilon_{0} \frac{\overline{N^{2}}}{N_{0}^{2}} \frac{<\zeta_{z}^{2}>^{2}}{<\zeta_{z G M}^{2}>^{2}} h\left(R_{w}\right) L(f, N)
$$

where

$$
L(f, N)=\frac{f \operatorname{arccosh}\left(\frac{\bar{N}}{f}\right)}{f_{30} \operatorname{arccosh}\left(\frac{N_{0}}{f_{30}}\right)}
$$

corrects for the latitudinal variability in the internal wave field. The expression for the finescale parameterization of $\epsilon$ depends on constants $N_{0}=5.24 \times 10^{-3} \mathrm{rad} / \mathrm{s}$ and $\epsilon_{0}=6.73 \times 10^{-10} \mathrm{~m}^{2} / \mathrm{s}^{-2}$. The function, $h\left(R_{w}\right)$ represents the internal wave field frequency content

$$
h\left(R_{w}\right)=\frac{1}{6 \sqrt{2}} \frac{R_{w}\left(R_{w}+1\right)}{\sqrt{R_{w}-1}}
$$

The values of $\epsilon_{\text {fine }}$ to $\epsilon_{\text {micro }}$ averaged over the duration of the US5 cruise show varying discrepancies as a function of depth (Fig. 2-6). Estimates of $\epsilon_{\text {fine }}$ are shown for $R_{w}=3$ and $R_{w}=7$, following values used in this region in previous studies (Whalen et al. (2012), Kunze et al. (2006)). The value of $R_{w}$, an estimate of the frequency content of the internal wave field, is a source of the discrepancy between previous estimates of dissipation in this region. The function $h\left(R_{w}\right)$, proportional to $\epsilon_{\text {fine }}$, is 2.7 times larger for $R_{w}=7$ versus $R_{w}=3$. Both choices of $R_{w}$ give values of $\epsilon_{\text {fine }}$ that exceed the observed values of dissipation from microstructure measurements.

In the deepest bins $\epsilon_{\text {micro }}<\epsilon_{\text {fine }}$. Turbulent mixing likely is due to breaking internal 


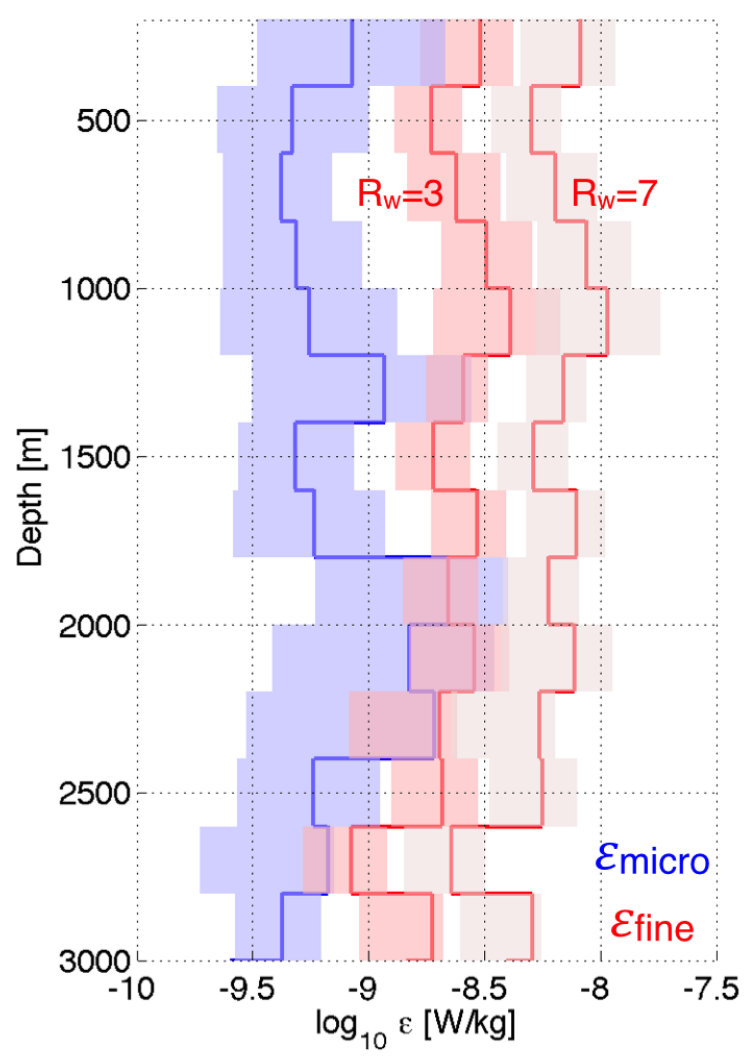

Figure 2-6: Microstructure estimates of dissipation (blue) compared to finestructure strain estimates (red). There are significant discrepancies between the two methods at all depths, with the largest signal between $500-1500 \mathrm{~m}$. Estimates of $\epsilon_{\text {fine }}$ are shown for values of $R_{w}=3$ and 7 .

lee-waves generated as frontal flows encounter rough topography. The microstructure data used here were collected predominately in the Polar Front over rough topography such that lee wave generation is expected. We note that the variability in $\epsilon_{\text {micro }}$ below $1500 \mathrm{~m}$ would be best studied in a height-above-bottom coordinate, to avoid aliasing shallow stations into mid-depths. Here we have removed stations with bottom depths shallower than $2000 \mathrm{~m}$. The strain parameterization assumes that the background wave field is GM, or spatially homogeneous and vertically isotropic, which may be violated in the presence of internal wave generation. The relevance of the strain parameterization in regions of the Southern Ocean with strong lee wave generation is an area of active research (Waterman et al. (2014)).

In the intermediate depth region (200-1000m) associated with interleaving features, the two estimates deviate the most significantly, with higher $\epsilon$ values obtained from the strain parameterization. We attribute the discrepancy to two issues. First, the 
fundamental assumption of the strain parameterization is that mixing is due to internal wave breaking. In the interleaving region, we show in chapter 3 that doublediffusive instability contributes to mixing rates (see also Merrifield et al. (2016)). Double-diffusive instabilities can only develop in regions of weak internal-wave shear. Stern et al. (2001) showed salt-fingers are resilient to shear processes at Richardson numbers (the ratio of the buoyancy frequency to the vertical velocity shear) greater than 0.5. Internal-wave shear may not be the dominant source of mixing in the interleaving region, which brings into question the use of the strain-parameterization in this portion of the water column.
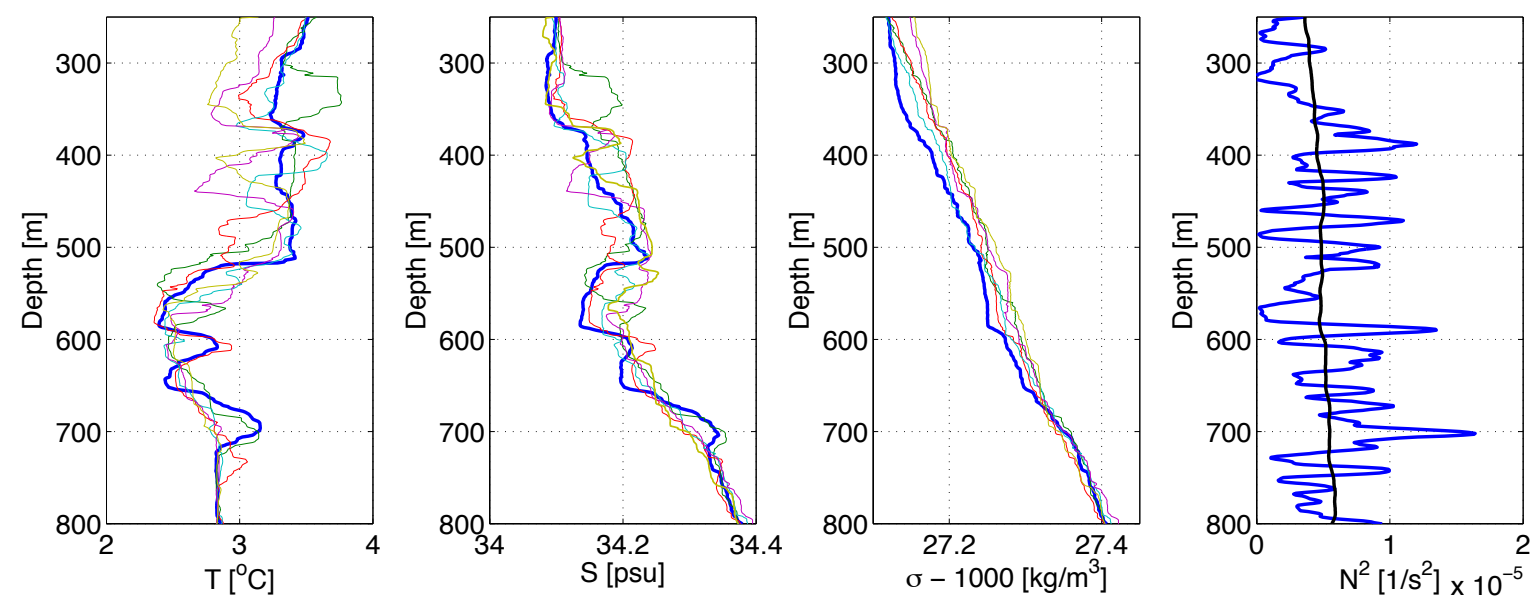

Figure 2-7: Profiles of temperature, salinity, potential density and buoyancy frequency from a 16 hour time series. A profile with density step like features near $600 \mathrm{~m}$ is shown in the thick blue line. The thick black line represents the background stratification in the fourth panel.

Second, the discrepancy between the values of $\epsilon_{\text {fine }}$ and $\epsilon_{\text {micro }}$ in the interleaving regime may be due to apparent strain, variability in $\zeta_{z}$ that is not associated with internal wave activity. Variability in an individual density profile can look step like like due to double-diffusive instabilities, with interfaces of steep-gradients separating wellmixed layers. This issue is discussed by Polzin et al. (2014) as a form of finestructure contamination. To illustrate this we show temperature, salinity, and potential density profiles from a 16 hour time-series of profiles taken in the Polar Front (Fig. 2-7). The thick blue line emphasizes one profile with a step-like feature just above $600 \mathrm{~m}$. The corresponding buoyancy frequency reflects aspects of the step structure with peaks associated with interface gradients and lower values in layers where the density profile varies weakly with depth. The vertical scales of the interleaving features range from tens to hundreds of meters. Estimates of internal wave strain as characterized by equation 2.2 , are sensitive to structures in the density profile, and can be biased high 
by apparent strain. In the depth bin centered on $600 \mathrm{~m}$, the value of $\epsilon_{\text {fine }}$ estimated from the thick blue line is as much as 6 times larger than $\epsilon_{\text {fine }}$ in the other profiles in the time series.

\subsection{Discussion}

This study suggests that the application of finestructure parameterizations in the Southern Ocean may lead to overestimates of mixing rates in some regions. In particular, we bring attention to frontal regions where water mass interleaving and thermohaline variance are enhanced. The Drake Passage region of the Southern Ocean is a convergence point for three climatological fronts: the Subantarctic Front, the Polar Front, and the Southern Antarctic Circumpolar Front. Baroclinic instabilities generate mesoscale eddies which strain the density field in this region, where strong temperature and salinity variability is observed both along and across isopycnals.

The application of the strain-based finestructure method to hydrographic data collected in Drake Passage shows significant deviations from microstructure based estimates. Values of turbulent dissipation estimated from a strain-based method are often an order of magnitude larger than rates based on microstructure measurements in the upper $1500 \mathrm{~m}$. We have identified two reasons for these deviations. First the assumption that internal wave shear is the dominant mixing process may not be valid in the interleaving regime. Recent work has suggested that double-diffusive instability contributes to mixing rates in this region of the water column (see Chapter 3, Merrifield et al. (2016)). Second, the strain variance is an integral over 40-100m vertical wavelengths, a characteristic scale of the interleaving features. Identifying internal wave strain using equation 2.2 is sensitive to thermohaline variability. This is particularly evident when a density profile has step-like features reflecting steep gradients and more homogenous layers of temperature and salinity. Studies such as Sloyan (2005) and Whalen et al. (2012) assume that vertical averaging and therefore large vertical length scales in individual density profiles are a reasonable estimate of the time-mean density field. This method relies on a scale separation between subinertial variability of the background stratification and smaller vertical length scales associated with internal wave activity. The premise of a scale separation is violated in the presence of density finestructure that is not due to internal wave activity. The Drake Passage region has significant watermass variability, particularly in the fronts, and 
it is unclear whether the time-mean density field is well estimated by an individual profile with vertical averaging.

Detecting isopycnal displacement and vertical advection associated with internal waves is difficult in the presence of lateral intrusions. Lateral intrusions may displace density surfaces introducing apparent strain. Alford and Pinkel (2000) defined an 'effective strain rate' by introducing a correction to the true vertical divergence, $\frac{\partial \mathrm{w}}{\partial z}$, to account for lateral advection of horizontal features (see (8) of Alford and Pinkel (2000)). The 'effective strain rate' may be related to the strain derived from the buoyancy frequency, $\zeta$, according to

$$
\frac{\partial \hat{\mathrm{w}}}{\partial z}=\frac{1}{\zeta} \frac{\partial \zeta}{\partial t}
$$

Similar corrections to the apparent strain may be feasible given sufficient spatial and temporal information of the vertical velocity field; however, such measurements are difficult to make in the field. In any case, we caution that strain-based parameterizations of dissipation based on hydrographic profiles should be evaluated carefully in regions of known intrusive activity.

In addition to the strain method, finestructure parameterizations based on velocity shear and density overturns have also been used in the Southern Ocean, many yielding large dissipation rates. Both shear-based and Thorpe scale estimates are sensitive to the resolution and noise of the observational dataset. Naveira Garabato et al. (2004)'s anomalously large dissipation estimates based on velocity shear are partially the result of noise levels in the ADCP-derived shear spectrum which become acute at wavelengths shorter than $50 \mathrm{~m}$. In the case of Naveira Garabato et al. (2004), the inclusion of noisy shear variance at short wavelengths biased their estimates high (Kunze et al. (2006)).

Thorpe scale estimation in the Southern Ocean is difficult because the stratification is weak compared to mid-latitude locations, and estimates of stability are sensitive to small errors in salinity. This is a significant issue in the Thompson et al. (2007) study, as they used expendable CTD instrumentation which have notoriously poor salinity resolution. As a result, their dissipation and diffusivity estimates based on Thorpe scales were biased high by up to two orders of magnitude (Frants et al. (2013)).

Assumptions that underpin finestructure parameterizations include: internal wave breaking drives diapycnal mixing, the background internal wave field can be described 
in relation to the Garrett and Munk spectrum, and finally that internal wave strain can be accurately diagnosed from hydrographic measurements. These assumptions are violated most significantly in the intrusive regime of the water column in the Southern Ocean, however, they are also violated in the presence of internal lee-wave generation near the seafloor. This study emphasizes the importance of obtaining additional direct measurements of turbulent dissipation to better understand the seasonal and spatial patterns of mixing in the Southern Ocean.

We have identified a host of uncertainties in both the physical assumptions of finestructure parameterizations and the methods used to implement them in the Southern Ocean. The relevance of finestructure parameterizations based on internal-wave driven physics in a regime where both internal wave activity and double-diffusion are active is unclear and is left for future work. This caveat is not particular to the Southern Ocean. In a global context, this study suggests that the dominant physical processes that drive mixing and existing parameterizations need to be revisited, especially in regions where thermohaline variance is pronounced. 


\section{Chapter 3}

\section{Enhanced diapycnal diffusivity in intrusive regions ${ }^{1}$}

\subsection{Introduction}

A number of processes in the Southern Ocean are thought to support high levels of mixing relative to other regions of the global ocean. At the surface, strong winds and storms force the ocean at near-inertial frequencies, generating internal waves that can propagate downward (Price, 1981). Upper ocean and mid-depth values of diapycnal diffusivity are believed to be set in part by the breaking of these nearinertial waves (e.g. Wu et al. (2011)). Deep reaching currents associated with density fronts flow over rough topography generating internal lee-waves which radiate energy and provide power for turbulence in the stratified ocean interior (e.g. Nikurashin and Ferrari (2010)). Watermass variability and strong mesoscale activity also precondition the water column for double-diffusive instability (e.g. Joyce et al. (1978)).

Due to the remoteness and harsh conditions, few direct measurements of mixing have been made in the Southern Ocean. A growing body of work has used hydrographic and velocity finescale data to estimate diapycnal mixing rates in this region (Naveira Garabato et al. (2004), Thompson et al. (2007), Sloyan et al. (2010), Wu et al. (2011),

\footnotetext{
${ }^{1}$ The text in this chapter has been accepted for publication as Merrifield, S. T., Laurent, L. S., Owens, B., Thurnherr, A. M., \& Toole, J. M. (2016). Enhanced Diapycnal Diffusivity in Intrusive Regions of the Drake Passage. Journal of Physical Oceanography, 46(4), 1309-1321. American Meteorological Society. Reproduced with permission.
} 
Whalen et al. (2012)). Thorpe scale analysis (Thorpe, 1977) and shear/strain based methods (Kunze et al. (1990)) are most commonly used to estimate microscale quantities. However, recent work has shown the potential for biases between mixing rates inferred from both of those methods and those from direct measurements. Mater et al. (2015) found Thorpe scale methods overestimate mixing rates when compared to direct measurements especially in the presence of large overturns. In the Southern Ocean, mixing rates inferred from finescale parameterizations are shown to overestimate those from microstructure measurements by as much as 1-2 orders of magnitude (Frants et al., 2013).

Previous estimates of mid-depth mixing rates based on finescale methods have been linked to both surface and bottom generated breaking internal waves. Naveira Garabato et al. (2004) attributed high values of diffusivity $\left(\kappa_{\rho} \sim 10^{-4} \mathrm{~m}^{2} \mathrm{~s}^{-1}\right.$ between $500-$ $1000 \mathrm{~m}$ ) to bottom-generated lee-waves. Multiple studies (Wu et al. (2011), Sloyan et al. (2010), Thompson et al. (2007), Kilbourne and Girton (2015)) suggest wind excited near-inertial waves radiate into the interior and break, contributing to high values of mixing below the surface layer. However, direct evidence of this process is still lacking. From these estimates of mixing rates, spatial and temporal patterns have been inferred. Thompson et al. (2007) conclude that the regions north and south of the Polar Front (PF) are dynamically different. Wu et al. (2011) suggested seasonality in the wind stress at the surface contributes to seasonality in mixing rates as deep as $1500 \mathrm{~m}$. Due to finescale biases in mixing rates from previously published studies, there is a renewed need to discuss the patterns and dynamics using direct measurements in this region.

The role of double-diffusive instability in driving mixing in the Southern Ocean has not been addressed using microstructure measurements. Studies focusing on the intrusive watermass characteristics in this region suggested that double-diffusive instability could be important to the dynamics (Joyce et al. (1978), Toole and Georgi (1981), You (2002)), but a comprehensive effort to study this using direct fine- and microstructure temperature $(\mathrm{T})$ and salinity $(\mathrm{S})$ has been missing. This work seeks to fill that gap by evaluating the density ratio, $R_{\rho}=\frac{\alpha \bar{T}_{z}}{\beta \bar{S}_{z}}$, where $g$ is gravity, $\rho$ is the surface density, $\alpha$ is the thermal expansion coefficient, $\beta$ is the haline contraction coefficient, $\bar{S}_{z}$ is the mean vertical gradient of salinity, and $\bar{T}_{z}$ is the mean vertical gradient of temperature. $R_{\rho}$ is a parameter which quantifies the favorability to double-diffusive instability. The two flavors of double-diffusive instability, salt-fingering and diffusive convection, can occur when $1<R_{\rho}<100$ and $0<R_{\rho}<1$, respectively. A narrower range of this 

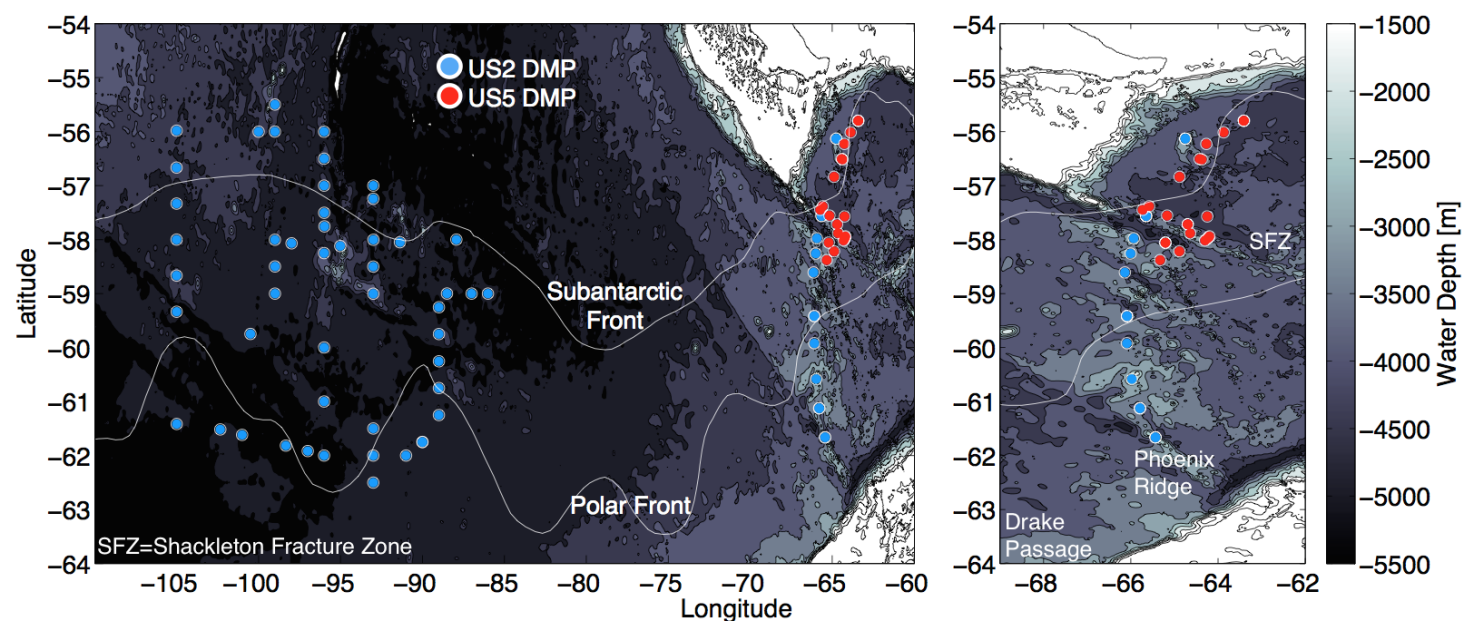

Figure 3-1: Map showing the microstructure stations taken during the US2 (blue) and US5 (red) cruises. The gray contours show water depth from Smith and Sandwell (1997). The white lines represent the climatological location of the Subantarctic and Polar Fronts, from Orsi et al. (1995).

ratio results in a growth rate that allows the instability to develop on a time scale comparable to the local buoyancy period $\left(\frac{2 \pi}{N}\right)$. This range has been estimated to be $1<R_{\rho}<2$ for salt fingering and $0.5<R_{\rho}<1$ for diffusive convection (Schmitt, 1979).

The Diapycnal and Isopycnal Mixing Experiment in the Southern Ocean (DIMES) has recorded more than $500 \mathrm{CTD}$ and 200 microstructure profiles with heavy sampling in Drake Passage. The direct measurements of dissipation made as part of the DIMES study are described by Ledwell et al. (2010), St. Laurent et al. (2012) and Sheen et al. (2014). These studies showed that enhanced internal wave fine structure and turbulence levels were not widespread, but limited to frontal zones where strong bottom currents collide with significant topography. In particular, St. Laurent et al. (2012) showed that velocity fine- and microstructure characteristics above the Phoenix Ridge are consistent with internal lee waves. There, the signal of enhanced dissipation extended to heights of $1500 \mathrm{~m}$ above the local ridge topography.

Here we present direct measurements of dissipation to study mid-depth values of $\epsilon$ and $\chi$. Values of dissipation are shown to be up to two orders of magnitude smaller than previously published values in the upper $1000 \mathrm{~m}$ inferred from finescale parameterizations. This work seeks to:

1. Report estimates of dissipation and diffusivity in the upper $1500 \mathrm{~m}$ of the Drake 
Passage region.

2. Present evidence that double-diffusion is a potentially important contributor to enhanced rates of thermal variance dissipation and vertical diffusivity.

3. Generate maps of the density ratio from hydrographic data to indicate regions of the Southern Ocean that may be susceptible to double-diffusive instability.

\subsection{Water mass variability and $\mathrm{T} / \mathrm{S}$ instrusions}

Water mass variability in the study region is high, as illustrated by hydrographic profiles from two stations in northern Drake Passage separated by $<14 \mathrm{~km}$. The two profiles were collected on separate cruises between the Polar and Subantarctic fronts and shed light on seasonal variability (Fig. 3-2a). Interleaving patterns in temperature (Fig 3-2b) and salinity (Fig 3-2c) are characteristic of the upper 200$1000 \mathrm{~m}$ throughout the region. These features are density-compensated and supported by mesoscale eddy activity and water mass variability. The buoyancy frequency (Fig $3-2 d$ ) peaks at the base of the mixed layer in US2 whereas the water column is weakly stratified during US5. Shallow mixed layers $(<100 \mathrm{~m})$ in the region are characteristic of Austral summer Dong et al. (2008). The water masses that contribute to the intrusive behavior in the upper $1000 \mathrm{~m}$ are Antarctic Surface Water (ASW), Upper Circumpolar Deep Water (UCDW), Winter Water (WW) and Antarctic Intermediate Water (AAIW).

The dissipation rate of turbulent kinetic energy (Fig. 3-2e) is elevated in the near surface and decays to background levels between 200-1000m. We attribute higher values of $\epsilon$ near $1500 \mathrm{~m}$, particularly during US5, to breaking internal waves that were generated at the seafloor. In contrast, the dissipation rate of thermal variance, $\chi$ (Fig. 3-2f) is enhanced at mid-depth, in the same depth range as strong $\mathrm{T} / \mathrm{S}$ intrusions. The highest values are found between $400-800 \mathrm{~m}$. The density ratio for the two profiles shown in Fig. 3-2g is computed using a 50 meter depth interval for the vertical gradients of $\mathrm{T}$ and $\mathrm{S}$. Based on the density ratio, we identify depth ranges in the temperature profile (Fig. 3-2h) that are favorable to salt fingering $\left(1<R_{\rho}<2\right)$

and diffusive convection $\left(0.5<R_{\rho}<1\right)$. The ranges of $R_{\rho}$ chosen correspond to growth rates that exceed the buoyancy period Schmitt (1994). The regions identified in Fig. $2 \mathrm{~h}$ correspond to enhanced values of $\chi$ in Fig. $2 \mathrm{f}$. 

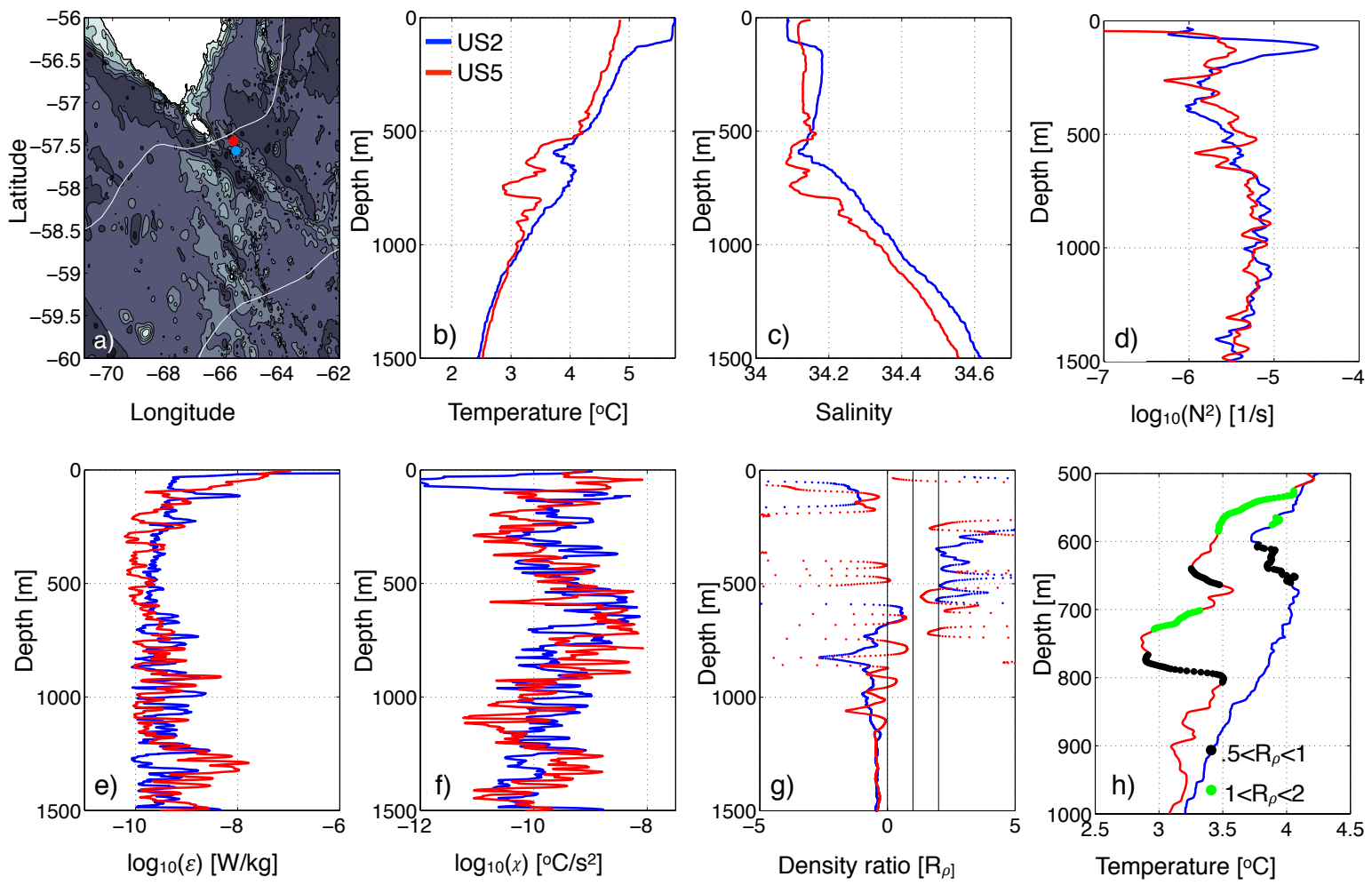

Figure 3-2: Blue (US2, Jan-Mar 2010) and red (US5 Oct-Nov 2013) stations within $14 \mathrm{~km}$ of each other. Profiles of temperature (b) and salinity (c) show evidence of intrusive behavior between $300-1000 \mathrm{~m}$. The stratification (d) is similar in each season with the exception of a peak at the base of the mixed layer in Austral summer (Dec-Mar). Turbulent dissipation rates, $\epsilon$ (e) and $\chi(\mathrm{f})$ show similar seasonal characteristics in magnitude and depth structure. $\chi$ is enhanced in the regions of strong $\mathrm{T} / \mathrm{S}$ interleaving. The density ratio, $R_{\rho}(\mathrm{g})$ shows values in the intrusive region of the water column consistent with both diffusive convection and salt fingering. A closer look at the temperature profiles (h) shows regions where diffusive convection is likely occuring (black), and similarly for salt-fingering (green).

\subsection{Turbulent quantities in the upper $1500 \mathrm{~m}$}

Mean estimates of the dissipation rates, $\epsilon$ and $\chi$, are shown in Fig. 3-3a and Fig. 3-3b, respectively. The profiles are averaged in 100 meter depth bins where the upper 200 $m$ are excluded to remove mixed layer and transition layer effects Sun et al. (2013). The shaded region represents the $95 \%$ confidence interval using a bootstrap method where the degrees of freedom is equal to the number of ten meter intervals in each bin. The ten meter length scale is chosen as an approximate value of the Ozmidov scale, $L_{o}=\sqrt{\epsilon / N^{3}}$, the eddy-overturning length scale in a stratified flow. 

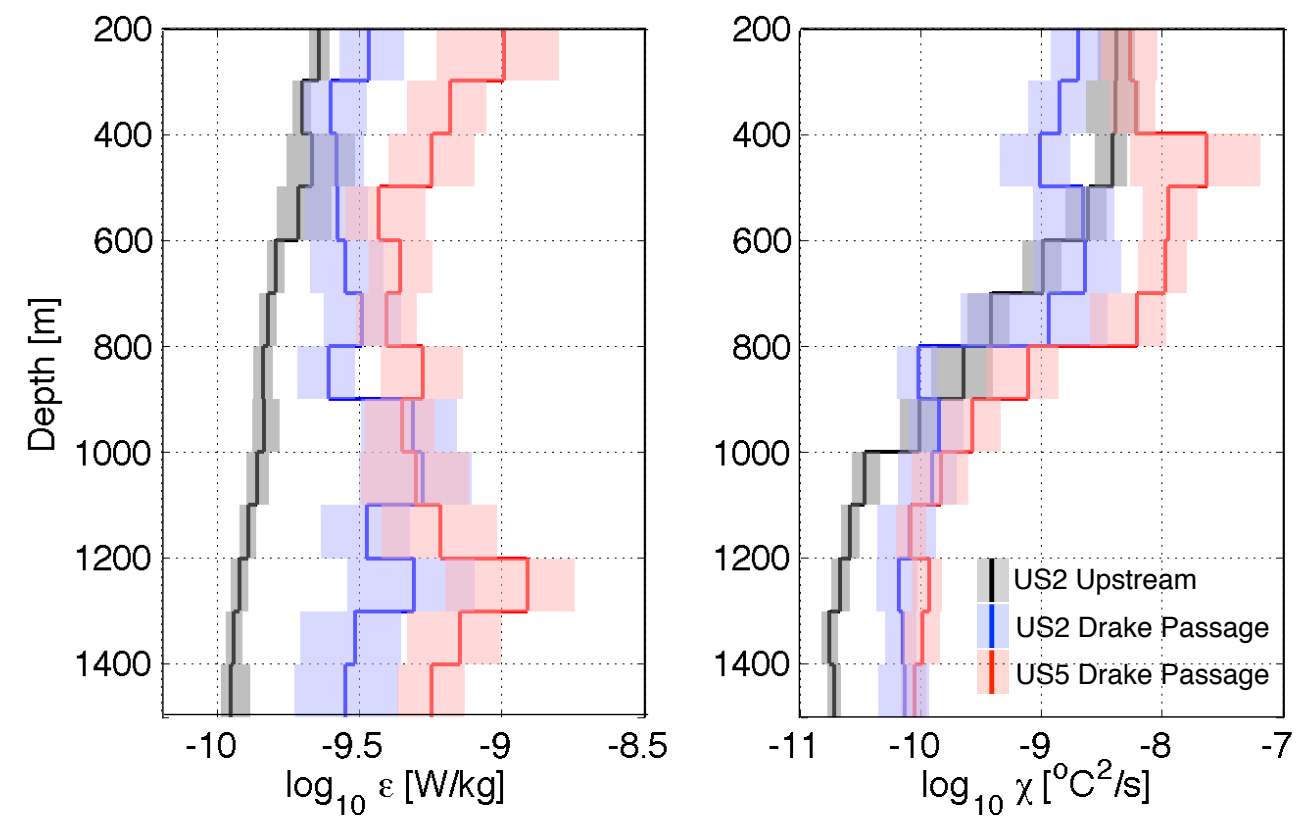

Figure 3-3: Averaged $\epsilon$ (a) and $\chi(\mathrm{b})$ as a function of depth. The colors represent different subsets of the data: US2 is split into upstream (SE Pacific) stations (black) and stations in Drake Passage (blue). Data from US5 are shown in red. The shaded regions represent error bars using a bootstrap method. The upper $200 \mathrm{~m}$ are not shown to remove the mixed layer and transition layer signal where stratification is highly variable and surface forcing has a significant impact. Averages are computed in 100 meter bins with stations whose bottom depth was shallower than $2000 \mathrm{~m}$ removed.

The average rates of turbulent kinetic energy dissipation $(\epsilon)$ range from $10^{-10} \mathrm{~W} / \mathrm{kg}$ upstream to $5 \times 10^{-10} \mathrm{~W} / \mathrm{kg}$ in Drake Passage. Mixing rates taken during the US5 cruise are higher at all depths between 200 and $1500 \mathrm{~m}$, suggestive of a seasonal difference between Austral summer (US2) and spring (US5). However, it is noted that US5 was concentrated in the Polar Front region while the US2 survey sampled farther south in Drake Passage. All mixing rates in Drake Passage, including those from US2, are higher in this depth range than those taken upstream. This is likely due to topographic roughness and convergence of the frontal jets in Drake Passage which support lee-wave activity and enhance the internal wave field at mid-depth. The thermal variance dissipation rate is enhanced between 200 and $1000 \mathrm{~m}$, with the largest values occurring in the US5 survey which was primarily conducted in the Subantarctic Front and Polar Front. The large water mass variability in this region preconditions the water column for double diffusive instabilities. 


\subsection{Statistics of dissipation at mid-depth sorted by density ratio and Richardson number}

To study the relationship between double-diffusive instability and the dissipation rates, $\epsilon$ and $\chi$, histograms of each are examined relative to the Richardson number and the density ratio. Only data in the depth range of $200-1000 \mathrm{~m}$ are used for this analysis. This eliminates surface and bottom boundary layer effects and isolates the signal associated with intrusive $\mathrm{T} / \mathrm{S}$ features. The Richardson number is defined as

$R i=\frac{N^{2}}{S h^{2}}$ where $S h^{2}=\frac{\partial u}{\partial z}+\frac{\partial v}{\partial z}^{2}$. Low Ri $(<0.25)$ is a necessary condition for (linear) shear instability resulting in turbulence and mixing. The probability density functions (PDFs) of $\epsilon$ and $\chi$ are sorted by the Richardson number, $R i$, and density ratio, $R_{\rho}$, (Fig 3-4). Statistics are done using the subset of microstructure profiles from the HRP2 because of the high vertical resolution velocity measurement from the MAVS. The Richardson number is computed over $10 \mathrm{~m}$ intervals to represent the Ozmidov scale. The density ratio, $R_{\rho}$, is computed from averages over 50 meter intervals. Stable density ratios (blue histograms) are associated with low values of $\chi$ at all values of the Richardson number. The histograms of $\epsilon$ show no significant trend when binned by the density ratio. The levels of $\chi$ are higher for values of $R i>0.25$ and $R_{\rho}$ in the range favorable to both diffusive convection and salt fingering. These patterns suggest that double-diffusive instabilities may be more important for mixing in the 500-1000m depth range than shear instabilities. We will explore this possibility in section $3 \mathrm{~d}$ in the context of vertical diffusivities.

\subsection{Methods of estimating vertical diffusivity}

Vertical diffusivity estimates typically invoke the Osborn (1980) method which is based on an energetic argument that steady-state, ensemble-averaged flow is in balance as $\langle P\rangle=\langle\epsilon\rangle+\left\langle J_{b}\right\rangle$ where $P$ stands for production of turbulent kinetic energy by the Reynolds stresses acting on the mean background shear. The viscous dissipation rate of turbulent kinetic energy $(\epsilon)$ and the increase in mean potential energy by the turbulent buoyancy flux $\left(J_{b}\right)$ represent two sinks of energy. Assuming a down-gradient eddy diffusion parameterization yields the vertical diffusivity,

$$
\kappa_{\rho}=\frac{\Gamma^{(t)}\langle\epsilon\rangle}{\left\langle N^{2}\right\rangle}
$$


$\mathrm{Ri}<.25$

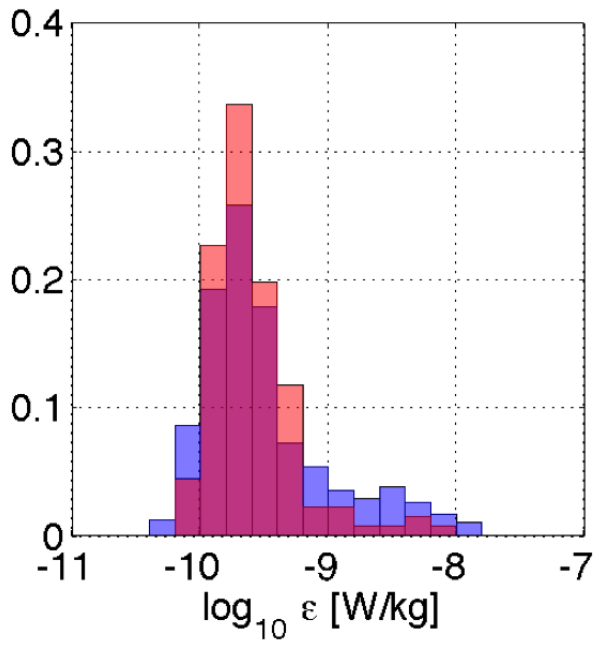

$\mathrm{Ri}<.25$

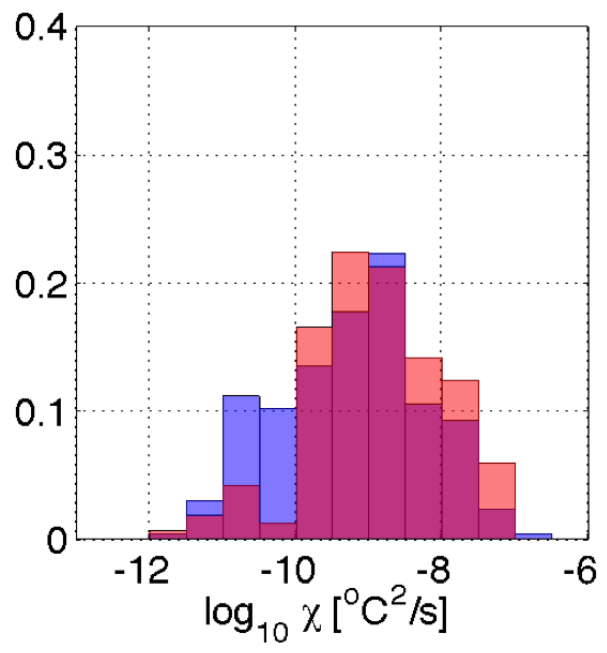

Ri $>.25$

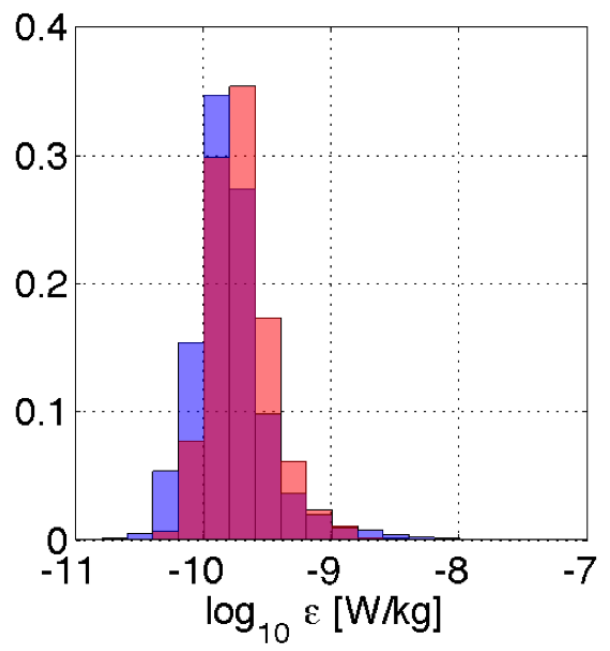

$\mathrm{Ri}>.25$

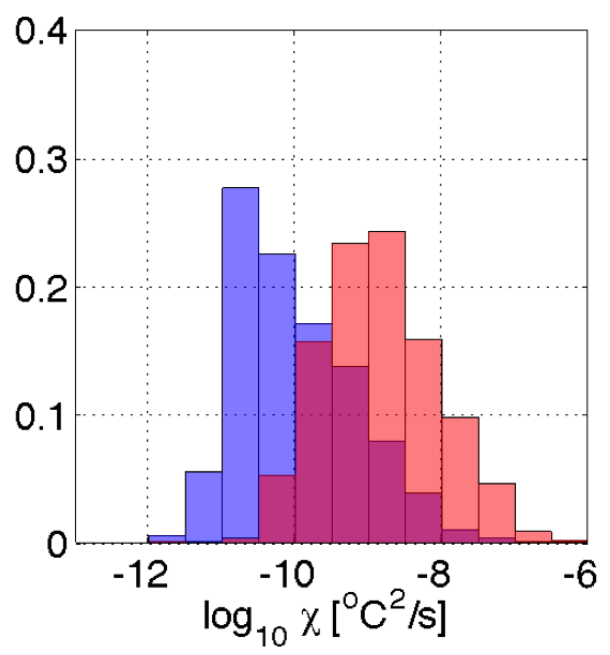

Figure 3-4: Probability density functions (PDFs) of $\epsilon(\mathrm{a}, \mathrm{b})$ and $\chi(\mathrm{c}, \mathrm{d})$ between $200-1000 \mathrm{~m}$ are sorted by density ratio and Richardson number. Statistics in blue represent a stable density ratio $\left(R_{\rho}<0\right)$, and those in red represent a regime strongly susceptible to diffusive convection and salt fingering $\left(0.5<R_{\rho}<2\right)$. Statistics of $\epsilon$ are similar for all values of $R_{\rho}$. PDFs show preference to high values of $\chi$ for $R_{\rho}$ in the double diffusive regime.

where $\Gamma^{(t)}$ is the ratio of potential energy gain to turbulent kinetic energy loss. This term is related to the mixing efficiency of turbulence and the scaled ratio of $\chi$ to $\epsilon$ (see below), and $\Gamma^{(t)}$ is often taken as a constant value of 0.2 . The averaging operations represented by $(\langle\cdot\rangle)$ are ensembles over many turbulent patches. A comprehensive discussion of these concepts is presented by St. Laurent and Schmitt (1999), and others. 
The Osborn and Cox (1972) method gives an alternate way of estimating vertical diffusivity based on the rate of molecular diffusion of scalar variance. The scalar in this study is temperature. The fundamental assumption in this method is that at the scale relevant to the estimation of $\chi$, the vertical fluxes dominate the variance production. Using a down-gradient eddy diffusion parameterization as in the Osborn (1980) method, diffusivity is estimated as

$$
\kappa_{T}=\frac{\langle\chi\rangle}{2\left(\frac{\partial \bar{T}}{\partial z}\right)^{2}}
$$

Using both methods and direct measurements of the turbulent quantities $\epsilon$ and $\chi$, vertical diffusivity is evaluated as a function of depth. The mean estimates are computed in $100 \mathrm{~m}$ bins shown in Fig 3-5 with blue curves for $\kappa_{\rho}$ and red curves for $\kappa_{T}$. The shaded regions represent the $95 \%$ confidence intervals based on a bootstrap method where the degrees of freedom are set by the number of twenty meter segments in each bin (a conservative estimate of degrees of freedom based on the larger estimates of the Ozmidov scale). Diffusivity is computed using all data from both expeditions (Fig. 3-5a) and the subset of data with a doubly stable density ratio $\left(R_{\rho}<0\right.$, Fig. $3-5 \mathrm{~b})$. The values of $\epsilon, \chi, T_{z}$ and $N^{2}$ are taken as ensemble averages over each $100 \mathrm{~m}$ bin. To strengthen the criterion for doubly stable statistics, the density ratio $R_{\rho}$ for that subset of the data was required to be less than zero on scales of $2 \mathrm{~m}, 5 \mathrm{~m}, 10 \mathrm{~m}$ and $25 \mathrm{~m}$. This eliminates the subjective nature of classifying a hydrographic segment based on scale-dependent gradients. To represent the enhanced temperature gradients associated with intrusions of various vertical scales, the $100 \mathrm{~m}$ ensemble averages of $\bar{T}_{z}$ are composed of gradients over scales of $5 \mathrm{~m}$.

In the region where $\mathrm{T} / \mathrm{S}$ intrusions are prevalent, the values of $\kappa_{T}$ exceed $\kappa_{\rho}$. Where there is no intrusive behavior notably at depths below $800 \mathrm{~m}, \kappa_{T} \approx \kappa_{\rho}$ (Fig 3-5c). If shear-driven turbulence is dominant, it is expected that the two methods for computing diffusivity will produce equal values. However, in the case of double-diffusive instability contributing to turbulent mixing, the Osborn (1980) model fails, as the concept of mixing efficiency becomes ill-defined St. Laurent and Schmitt (1999). Thus, the Osborn and Cox (1972) method provides the reliable means for estimating diffusivity for double-diffusive scenarios. This is validated further by the result that $\kappa_{T} \approx \kappa_{\rho}$ for data in the intrusive depth range but with doubly stable density ratio (Fig 3-5b) . This is consistent with Stern et al. (2001) who showed that for Richardson numbers $>0.5$, salt fingers are resilient to shear processes. For this subset of the 

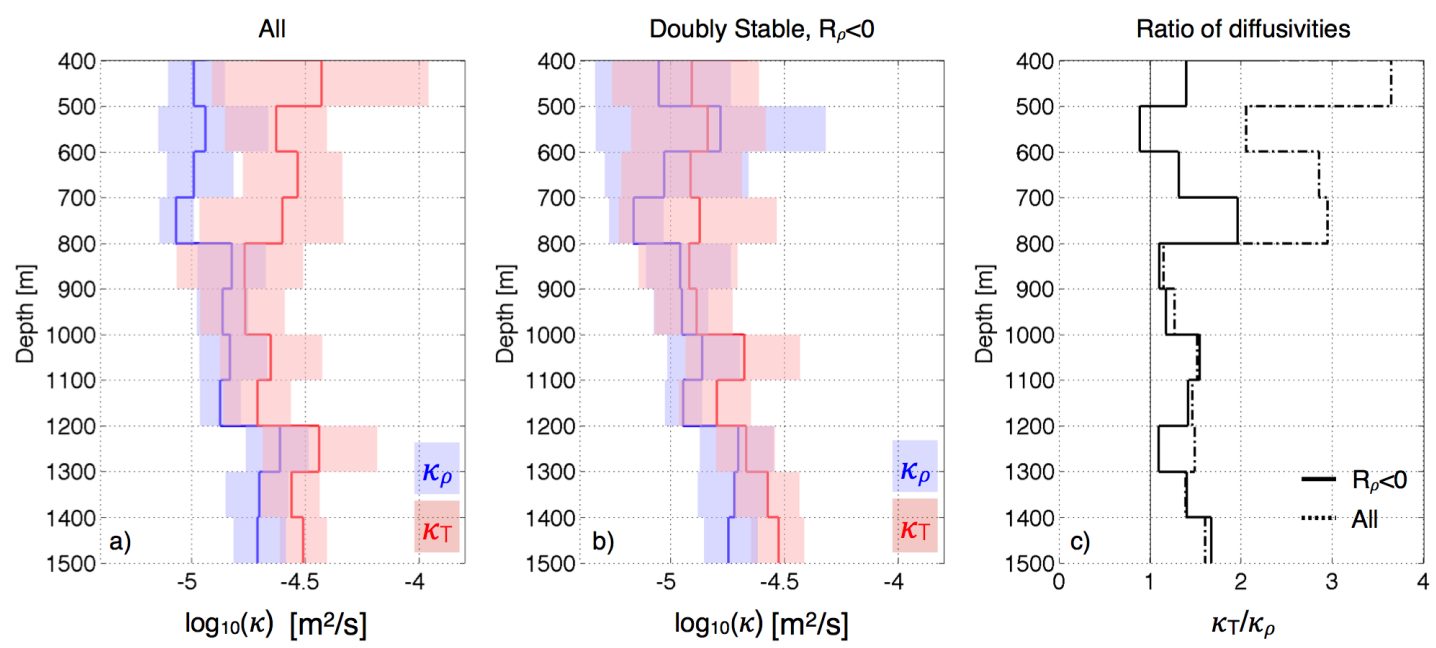

Figure 3-5: Diffusivity as a function of depth computed using a) all data during the two expeditions and b) only data in the doubly stable regime, where $R_{\rho}<0$. The data are averaged in $100 \mathrm{~m}$ bins using the Osborn (1980) method $\left(\kappa_{\rho}\right.$, blue, a and b) and the Osborn and Cox (1972) method ( $\kappa_{T}$, red, a and b). In the depth range associated with $\mathrm{T} / \mathrm{S}$ intrusions $(400-800 \mathrm{~m})$, the values of $\kappa_{T}$ exceed $\kappa_{\rho}$ when all data are used. When data in the intrusive regime but with a doubly stable density ratio are used to compute diffusivity, this signal is eliminated and $\kappa_{T} \approx \kappa_{\rho}$. The ratio of c) $\kappa_{T} / \kappa_{\rho}$ as a function of depth is shown for the doubly stable regime (solid) and all data (dashed).

data, the mesoscale eddy field is active, the water column is not susceptible to double diffusion, and the two methods for computing diffusivity yield the same value. The ratio of $\kappa_{T}$ to $\kappa_{\rho}$ (Fig. $3-5 \mathrm{c}$ ), for all data (dashed) show that $\kappa_{T}$ exceeds $\kappa_{\rho}$ by a factor of 3 in the intrusive regime. The ratio of diffusivities for the doubly stable data (solid) is less than 1.5 and converges with the ratio for all data below the intrusive regime $(>800 \mathrm{~m})$.

\subsection{Spatial patterns in intrusive regions}

It has been shown using direct measurements that the dissipation rate of thermal variance is enhanced in intrusive regions of the water column. With limited measurements from microstructure profilers, hydrographic data are used to study large-scale spatial patterns of the density ratio in this region. Using temperature and salinity data from CTD casts, the density ratio is computed in $50 \mathrm{~m}$ depth intervals for three meridional sections at $93^{\circ} W$ (Fig. 3-6a) and in the Drake Passage (Fig. 3-6b,c). 

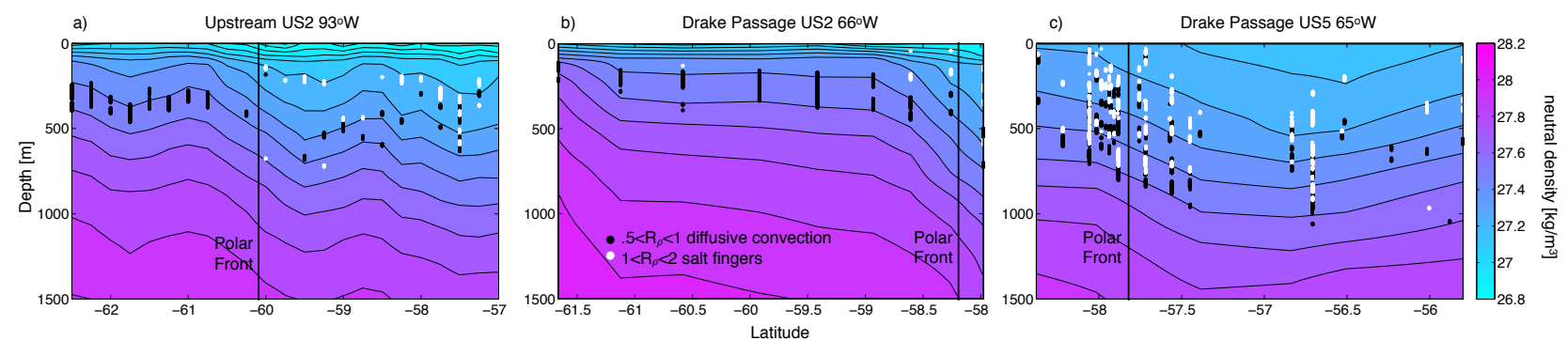

Figure 3-6: Three meridional transects of neutral density (colors) at a) $93^{\circ} \mathrm{W} \mathrm{b)} 66^{\circ} \mathrm{W}$ and c) $65^{\circ} \mathrm{W}$. The circles represent regions in the water column where the density ratio $R_{\rho}$ is between $0.5-1$ (black) and 1-2 (white). These ranges represent potential for double-diffusive instabilities with growth rates comparable to the buoyancy frequency $(\mathrm{N})$, the timescale associated with internal wave-driven turbulence. The density ratio is preferable to double-diffusive instability in neutral density classes between 27.2 and $27.7 \mathrm{~kg} / \mathrm{m}^{3}$. The Polar Front in each panel is defined by the hydrographic measurements made during the respective cruises, US2 (a and b), US5 (c).

The region of the water column susceptible to double diffusive instability corresponds to density classes between 27.2 and $27.7 \mathrm{~kg} / \mathrm{m}^{3}$. South of the Polar Front, the density ratio is predominately in the range favoring diffusive convection. North of the PF and in the frontal region, salt fingering becomes more prevalent. This pattern south of the $\mathrm{PF}$ is likely due to the temperature inversion that exists between 100-300 $\mathrm{m}$ as winter water subducts. Because the isopycnals deepen from south to north through Drake Passage, the potential for double diffusion extends deeper into the water column in the Polar Front and Subantarctic Front.

\subsection{Discussion}

It has been difficult to assess values and patterns of subsurface mixing due to a lack of direct observations in the Southern Ocean. Turbulent quantities are important to the energy balance of the global ocean and are prevalently parameterized in largescale circulation models. Results from previous work using finestructure methods to interpret mixing rates have recently been shown to be biased high (Frants et al. (2013), Mater et al. (2015), Waterman et al. (2014)), often by up to two orders of magnitude. This work has revisted the values and patterns of mid-depth mixing in this region to inform modeling efforts and large-scale estimates of vertical diffusivity. Even with double-diffusive enhancement of $\kappa_{T}$ relative to $\kappa_{\rho}$, our estimates are still typically an order of magnitude less than values of diffusivity estimated from finestructure 
methods.

Intrusive features in the water column are associated with statistics of $\epsilon$ and $\chi$ which, when sorted by the density ratio and Richardson number, show a prevalence toward high values of $\chi$ for $R i>1$ and $0.5<R_{\rho}<2$. This corresponds to the highly doublediffusive favorable regime described by Schmitt (1994) and St. Laurent and Schmitt (1999), where double-diffusive growth rates are comparable to the local buoyancy frequency. The potentially important role of double-diffusive processes in enhancing mixing rates has long been suggested for this region of the Southern Ocean (Joyce et al. (1978), Toole and Georgi (1981), Thompson et al. (2007)).

To study interactions between the large, intermediate and dissipative scale, we consider the triple decomposition, proposed by Stern (1967). This approach has been used in various forms by other investigators (Joyce (1977); Davis (1994); Garrett (2001); Ferrari and Polzin (2005)). Here, we use the formulation of Joyce (1977), who specifically considered the case of intrusions occupying the intermediate scale between the large scale mean (dominated by eddies) and the turbulent scale where dissipation occurs. The velocity and temperature parameters are taken to be the sum of contributions from the three scales: $\phi=\bar{\phi}+\tilde{\phi}+\phi^{\prime}$, where the $(\cdot),(\tilde{\cdot})$, and $(\cdot)^{\prime}$ denote the large, intrusive and dissipative scales, respectively. The statistics on these disparate scales are assumed to be uncorrelated such that averaging operations obey $\overline{\tilde{\phi}}=\widetilde{\phi}^{\prime}=0$. This particular assumption is equivalent to asserting there is a spectral gap between the classes of processes involved, which is impossibe to justify formally. As discussed by Davis (1994), the value of the triple decomposition may be more pedagogical than literal. Here, we present the formulation of thermal variance to establish a framework connecting the dissipative processes to the larger scale system of the Southern Ocean.

Under these assumptions, the steady state, non-divergent thermal variance balances connecting the intermediate to the large scale, and the small to the intermediate scale, become:

$$
\begin{array}{r}
\langle\tilde{\mathbf{u}} \tilde{T}\rangle \cdot \nabla \bar{T}-\left\langle\widetilde{\mathbf{u}^{\prime} T^{\prime}} \cdot \nabla \tilde{T}\right\rangle=0 \\
\left\langle\widetilde{\mathbf{u}^{\prime} T^{\prime}} \cdot \nabla \tilde{T}\right\rangle+\left\langle\mathbf{u}^{\prime} T^{\prime}\right\rangle \cdot \nabla \bar{T}=-\frac{1}{2}\langle\chi\rangle
\end{array}
$$

where $\widetilde{(\cdot)}$ represents an ensemble average over scales large compared to the dissipative scale and small compared to the intermediate scale, and $\langle\cdot\rangle$ represents an ensemble 
average over scales large compared to the intermediate scale and small compared to the large scale. Ferrari and Polzin (2005) employ these same equations, but label the intermediate scale as being attributable to mesoscale eddies rather than intrusions. The representation of intrusions as the intermediate scale is consistent with the original formulations of Stern (1967) and Joyce (1977). Joyce (1977) specifically discussed (3.3) in the context of the variance production of the intrusive scale balancing that of the larger scale. Substituting (3.3) into (3.4) gives:

$$
\langle\tilde{\mathbf{u}} \tilde{T}\rangle \cdot \nabla \bar{T}+\left\langle\mathbf{u}^{\prime} T^{\prime}\right\rangle \cdot \nabla \bar{T}=-\frac{\langle\chi\rangle}{2} .
$$

As described by Ferrari and Polzin (2005), it is conventional to represent the terms on the left-hand-side as diffusion terms. The first term, representing the variance production of intermediate scale, is taken as a lateral (isopycnal) eddy flux acting on the lateral temperature gradient, such that $\langle\tilde{\mathbf{u}} \tilde{T}\rangle \cdot \nabla_{\text {iso }} \bar{T}=-K_{e}|\nabla \bar{T}|^{2}$, where $K_{e}$ represents a diffusivity for eddy stirring. The second term, representing the variance production by the turbulent scale, is taken as a vertical (diapycnal) turbulent flux acting on the vertical temperature gradient, such that $\left\langle\mathbf{u}^{\prime} T^{\prime}\right\rangle \cdot \nabla \bar{T}=-\kappa_{T} \bar{T}_{z}^{2}$. Used in (3.5), these give

$$
K_{e}\left|\nabla_{i s o} \bar{T}\right|^{2}+\kappa_{T} \bar{T}_{z}^{2}=\frac{\langle\chi\rangle}{2}
$$

This expression may be thought of as a generalized version of the Osborn and Cox (1972) relation (3.2), with the thermal variance production now being shared by the intermediate scale, as represented by the $K_{e}$ term, and the turbulent scale. Barring some additional information, it is not possible to calculate the diffusivity parameters $\left(K_{e}, \kappa_{T}\right)$ in (6) from measured data. Notably, this study shows that replacing $\kappa_{\rho}$ from (3.1) with $\kappa_{T}$ is not an acceptable choice in an intrusive watermass where double-diffusive processes are active. Alternatively, if independent estimates of $K_{e}$ are available, they could be used in (3.6) to estimate $\kappa_{T}$ from estimates of $\langle\chi\rangle$. Lacking such additional information the problem is underdetermined, so we study the limits of the balance by evaluating the upper bound of each term separately. For this assessment, we will use data from US5, as it comes from a small region of the frontal zone where the hydrography does not vary widely by latitude, and the sample grid allows us to estimate lateral gradients on the mesocale. 
First, we study the limit where $K_{e}$ term can be neglected in (3.6) and estimate $\kappa_{T}$. As an alternative to the estimates presented in the previous section, we base our estimates here using the scale of the intrusions as defining the vertical scale (typically 50 to $100 \mathrm{~m}$ ) for the estimates of $\bar{T}_{z}$. We also estimate the buoyancy gradient, $N^{2}$, for use in comparative estimates of $\kappa_{\rho}$ from (3.1). To accurately estimate vertical gradients in the intrusions, the density is sorted to the minimum potential energy state Winters and D'Asaro (1996) over each intrusion. This removes any bias incurred by an arbitrary choice of vertical scale. The vertical gradient of temperature is done as a linear regression. The ensemble average is done over $0.05 \mathrm{~kg} / \mathrm{m}^{3}$ bins of neutral density, and the resulting $\kappa_{\rho}$ and $\kappa_{T}$ estimates are given in Fig 3-7. Errorbars are consistent with bootstrap esimates from Fig. 5. Data are used from US5 in the density bins associated with intrusions with criteria that the intrusions are at least $5 \mathrm{~m}$ in length and that the density ratio is in the range of $0.5<R_{\rho}<2$. This isolates the signal in the intrusive regime of features that are potentially doubly-diffusive. In this range, $\kappa_{T}>\kappa_{\rho}$ further corroborating the evidence that double-diffusive instabilities are contributing to enhanced levels of diapycnal diffusivity.

Next, we study the limit of the maximum potential role of eddy stirring in supporting thermal variance production by neglecting the $\kappa_{T}$ term in (3.6) and estimating $K_{e}$. This gives

$$
K_{e} \sim \frac{\langle\chi\rangle}{\left\langle 2\left|\nabla_{i s o} \bar{T}\right|^{2}\right\rangle}
$$

Using (3.7) with objectively mapped isopycnal temperature gradients from the US5 dataset over scales greater than $100 \mathrm{~km}$, we estimated the parameter $K_{e}$ as shown in Fig 3-8. The values inferred by $(3.7)$ are $O\left(10^{3}-10^{4} \mathrm{~m}^{2} / \mathrm{s}\right)$ in the intrusive regime.

Our estimates of $K_{e}$ are an upper bound, representing the limit where the variance budget is closed between mesoscale stirring along isopycnals and the thermal variance dissipation. This is extremely unlikely, as variance production by vertical mixing must play a role. To assess the relative role of eddy stirring relative to vertical mixing, we consider previous estimates of $K_{e}$ from modeling studies. High-resolution modeling of the ACC system has been considered by a number of investigators (Abernathey et al. (2010), Ferrari and Nikurashin (2010), Klocker and Abernathey (2014)). Estimates for the general ACC system show $K_{e}$ in the range $O\left(10^{2}-10^{3} \mathrm{~m}^{2} / \mathrm{s}\right)$. Ferrari and Nikurashin (2010) specifically considered the case of eddy stirring in the frontal jet 


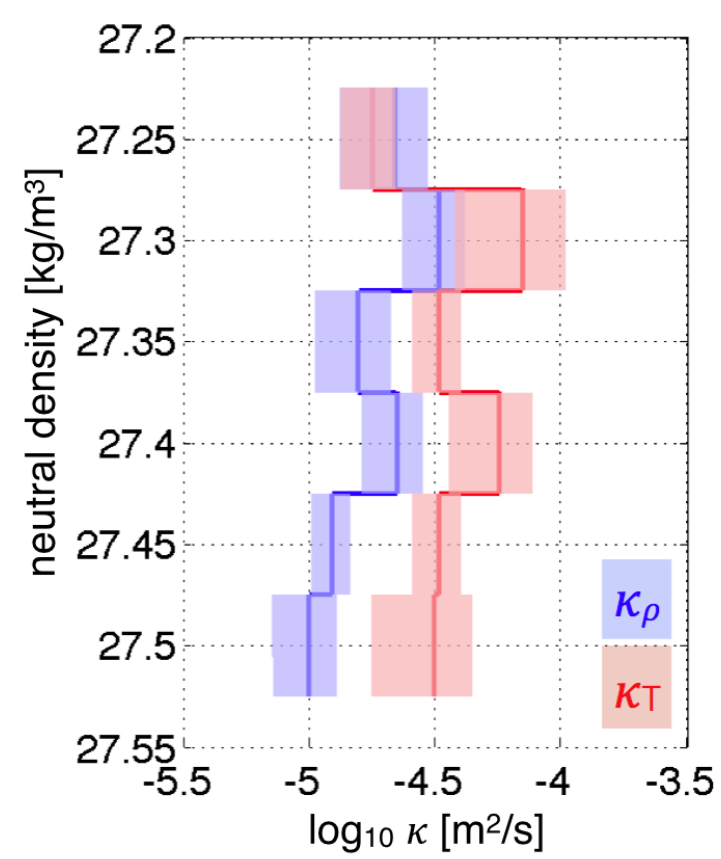

Figure 3-7: The values of $\kappa_{\rho}$ (blue) and $\kappa_{T}$ (red) in $0.05 \mathrm{~kg} / \mathrm{m}^{3}$ neutral density bins using the US5 dataset. A linear regression over each intrusion is used to compute values of $\left|T_{z}\right|$ and $N^{2}$. The density is first sorted to the minimum potential energy state before calculating the buoyancy frequency. Only bins of neutral density, $\gamma$, that included intrusions are shown and the values of $\kappa_{T}$ and $\kappa_{\rho}$ are computed using intrusion-scale quantities averaged in each bin. Additionally, only intrusions that satisfy the criterion of $0.5<R_{\rho}<2$ are shown to isolate the signal of features that are potentially doubly-diffusive.

of the ACC, describing supression of $K_{e}$ values in the core of the frontal zone. There, values of $K_{e}$ are predicted to be $O\left(10^{1}-10^{2} \mathrm{~m}^{2} / \mathrm{s}\right)$. Recently, Naveira Garabato et al. (2016) used data-based estimates, showing $K_{e}$ values of $O\left(10^{1} \mathrm{~m}^{2} / \mathrm{s}\right)$ in the core of the frontal zone just east of Drake Passage, further supporting the supression of isopycnal stirring in the upper $1000 \mathrm{~m}$ of the frontal zones in this region.

Data from the US5 expedition used in this study was taken in the Subantarctic Front and Polar Front, such that supressed levels of eddy mixing described by Ferrari and Nikurashin (2010) and Naveira Garabato et al. (2016) are expected. Thus, our estimates from (3.7) are seemingly $\mathrm{O}(2)$ to $\mathrm{O}(3)$ times larger than these studies suggest for the eddies in this region. This suggests that the variance production necessary to support our observations of $\langle\chi\rangle$ must predominantly come from vertical mixing, suggesting 


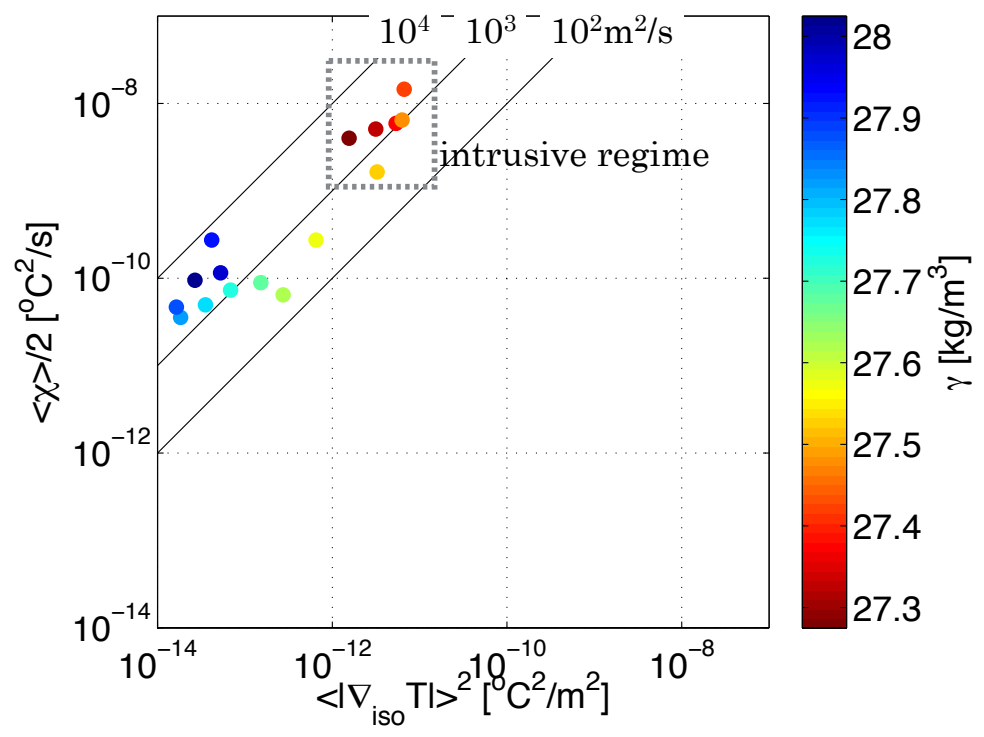

Figure 3-8: Ensemble averages in $0.05 \mathrm{~kg} / \mathrm{m}^{3}$ neutral density bins are taken of $\langle\chi\rangle / 2$ and $\left\langle\left|\nabla_{\text {iso }} T\right|\right\rangle^{2}$ to study the limit of the thermal variance budget where vertical dissipation is neglected. In this limit, the value of $K_{e}$, taken as upper bound, is generally larger than $10^{3} \mathrm{~m}^{2} / \mathrm{s}$. The black lines represent lines of constant $K_{e}$ of $10^{2}, 10^{3}$, and $10^{4} \mathrm{~m}^{2} / \mathrm{s}$

$$
\frac{2 \kappa_{T} \bar{T}_{z}^{2}}{\langle\chi\rangle} \approx 1+O\left(10^{-2}\right) .
$$

In this statement, the impact of the intrusive watermass is crucial for elevating $\kappa_{T}$ relative to mixing levels that would occur in the absence of double diffusion. If double diffusive instability is driving enhanced dissipation in the intrusive layers, the mixing efficiency of $\Gamma^{(t)}=0.2$ used in the Osborn (1980) model is invalid. To assess the failure of the mixing efficiency model in the intrusive watermass, we estimate the dissipation ratio,

$$
\Gamma=\frac{\langle\chi\rangle\left\langle N^{2}\right\rangle}{2\langle\epsilon\rangle\left\langle T_{z}\right\rangle^{2}} .
$$

In the case of turbulent mixing, the dissipation ratio is related to the mixing efficiency of turbulence, and generally has a value of $\Gamma=\Gamma^{(t)} \approx 0.2$. In the case of doublediffusive driven mixing, the dissipation ratio elevates, typically to values between 0.5 and 1 St. Laurent and Schmitt (1999). It follows that estimates of the dissipation ratio are used to identify double diffusive processes. The dissipation ratio for the intrusive regions of the water column in the US5 data, (Fig. 3-9), shows elevated values of $\Gamma$ consistent with double-diffusive processes. We therefore accept the Osborn and Cox 


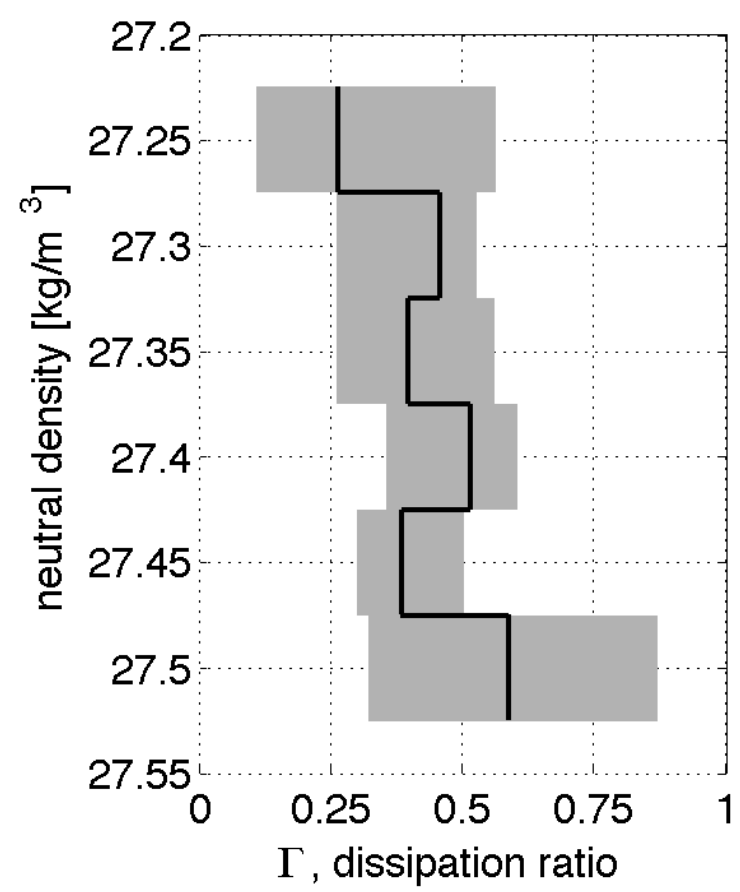

Figure 3-9: Values of the dissipation ratio, $\Gamma$, using intrusion scale quanitites that satisfy $0.5<R_{\rho}<2$. The values are consistently higher than 0.2 in the intrusive regime.

(1972) based estimate of $\kappa_{T}$ as the most meaningful estimate of mixing rate for the intrusive watermass region.

Our results indicate that it may be necessary to resolve the watermass finestructure to account for diffusive mixing in the Southern Ocean. The vertical scales of intrusions are typcially in the 50 to $100 \mathrm{~m}$ range, and the gradients of temperature and salinity on these scales are the link between the mesoscale variance production and the microscale variance dissipation. While these scales are too fine to be resolved in climate models, they are resolved in global-scale process models that are used to examine the Southern Ocean (Abernathey et al. (2010), Klocker and Abernathey (2014)). Our estimates of $\kappa_{T}$ could be used to prescribe diffusivities acting in the Southern Ocean intrusive watermasses of these models. Process studies with the high-resolution models could then be used to pursue parameterizations for models with courser, non-watermass resolving, resolutions. This approach, based on a synergy between measured data and processes oriented numerical simulations, will likely be the most successful mechanism for parameterizing sub-gridscale physics in climate models. 
The waters of the Southern Ocean have long been established to be the site of enhanced mixing. Attention has focused on the role of surface forcing, via the mixed layer, at supporting enhanced internal wave processes in the upper ocean (Dong et al. (2008), Kilbourne and Girton (2015)). Still more attention has examined the role of lee waves at driving deep turbulence levels (e.g., St. Laurent et al. (2012)). At intermediate depths where intrusive watermasses are present, we contend that double diffusive processes are an important source of enhanced dissipation in this region, which generally is not accounted for in large-scale models. While estimates of mass transport are often insensitive to the particular details of how ocean processes are parameterized, the watermass characteristics of temperature and salinity can vary radically. As small changes in temperature and salinity properties evolve over long model simulation integrations, the watermass properties can diverge from reality, destroying the ability of such models to forecast thermodynamic budgets. As models will never be able to inform us of the subgridscale processes that drive ocean mixing, it is therefore necessary to use the data provided from direct measurements to assess the potential roles for turbulence, double diffusion, and lateral stirring. 


\section{Chapter 4}

\section{Mixed layer processes and boundary layer turbulence}

\subsection{Introduction}

Ocean mixed layer (OML) processes influence the transfer of heat, momentum, and tracers between the atmosphere and the ocean, and have important implications in climate studies. Water mass properties may be modified in the OML, which when subducted into the ocean interior affect the stratification and consequently the large scale ocean circulation. The OML is an area of active study, and recent work has shown the thickness of the OML in the Southern Ocean is biased shallow in climate models (Sallée et al. (2013)). In particular, in the Drake Passage region, these errors may exceed 90\% (Belcher et al. (2012)).

Surface boundary layer parameterizations have been developed to provide simplified descriptions of the important dynamical processes in the OML. In most global climate models, turbulence in the OML is driven by surface wind stress and atmospheric fluxes. Scalings for the structure and magnitude of the turbulent kinetic energy (TKE) dissipation rate in the OML are derived from atmospheric experiments (Lombardo and Gregg (1989)). The structure of the turbulence can be defined by a stability parameter $\zeta=\frac{z}{L}$ (Monin and Obukhov (1954)). The Obukhov length scale (Obukhov (1946)), $L$, is defined as:

$$
L=\frac{-u^{* 3}}{\kappa J_{b}}
$$


where $\kappa$ is the Von Karmen constant $(=.41)$ and $u^{*}=\sqrt{\frac{\tau}{\rho}}$ is the friction velocity. The buoyancy flux, $J_{b}$ is defined as:

$$
J_{b}=\frac{g}{\rho}\left[\frac{\alpha}{c_{p}} Q_{t o t}+\left(\beta \frac{s}{1-s} L_{E}\right) Q_{l}\right]
$$

where $g$ is gravity, $\rho$ is the surface density, $\alpha$ is the thermal expansion coefficient, $C_{p}$ is the specific heat of seawater, $\beta$ is the haline contraction coefficient, $s$ is salnity in concentration units, $Q_{t o t}$ is the sensible heat flux, $Q_{l}$ is the latent heat flux, and $L_{E}$ is the latent heat of evaporation. The Obukhov length describes the static stability of the surface layer. When $L<0$, the surface is statically unstable and under neutral conditions, $L \rightarrow \infty$.

The stability parameter, $\zeta$, determines whether winds or buoyancy forcing are the dominant source of turbulent mixing in the boundary layer at depth $z$. If $\zeta<<1$, wind forcing is dominant and if $\zeta>>1$, the buoyancy forcing is dominant. In these limits, the dissipation of turbulent kinetic energy (TKE) has been shown to scale according to:

$$
\begin{array}{rlrl}
\epsilon \sim \epsilon_{s} & =\frac{u^{* 3}}{\kappa z} & & (\zeta<<1) \\
\epsilon \sim J_{b} & & (\zeta>>1)
\end{array}
$$

For intermediate cases, an empirical relationship has been proposed by Lombardo and Gregg (1989):

$$
\epsilon \sim .58 J_{b}+1.76 \epsilon_{s}
$$

Many recent studies have questioned the validity of the scalings (4.3-4.5) for the OML, particularly since they originally were developed for the boundary layer at the atmosphere-land interface. Unlike the solid lower boundary of land masses, the atmosphere-ocean interface is a deformable surface. Both observational (D'Asaro et al. (2014), Sutherland et al. (2014)) and modeling studies (Kukulka et al. (2009), Fox-Kemper et al. (2011)) have shown that the depth and structure of the OML are poorly reproduced by these classical parameterizations in part due to the effects of surface waves. An empirical validation of (4.5) has been carried out by Lombardo and Gregg (1989) in the low wind regime (wind speeds less than $10 \mathrm{~m} / \mathrm{s}$ ) with reasonable agreement. In the Southern Ocean where strong mean zonal winds energize the wavefield throughout the year, the validity of the scalings (4.3-4.5) has not been tested. 
This chapter analyzes recently collected measurements from the DIMES project (see section 1.5 for a description of the data used here) to study the structure and magnitude of dissipation in OML. In section 4.2, we describe the seasonal structure of the OML and present the high-frequency response to storm events using in-situ turbulence and shipboard meteorological measurements. In section 4.3 we consider the classical scalings based on surface wind stress and atmospheric fluxes (4.3-4.5) and show that during storm conditions, these scalings underestimate the dissipation of TKE in the wave-driven near-surface layer, and overestimate the dissipation below. The inability of the classical scalings to capture the vertical dependence of the TKE dissipation during strong wind events suggests that the surface wave field likely affects the dynamics of the OML. More detailed observations are required to assess the affects of the surface wave field on the OML in this region. We conclude with a discussion of two alternative sources of boundary layer turbulence that are potentially important in the Southern Ocean, Langmuir turbulence and submesoscale instabilities. These processes have been analyzed by D'Asaro (2014) and Thomas et al. (2013), respectively, in regions with similar conditions to the Southern Ocean with more comprehensive data sets.

\subsection{Mixed layer structure}

The OML is defined as the region of the water column from the surface to a depth $H_{\rho}$ that has uniform density and enhanced vertical mixing. The mixed layer depth, $H_{\rho}$, is defined as the depth at which hydrographic properties are homogenous using

a density criterion of $0.05 \mathrm{~kg} \mathrm{~m}^{-3}$. We also define the actively mixing layer, $H_{\epsilon}$, the depth at which $\epsilon$ falls to a background level of $10^{-9} \mathrm{~W} / \mathrm{kg}$ (following Brainerd and Gregg (1995) and Sutherland et al. (2014)). The actively mixing layer has implications for biological processes such as phytoplankton blooms and the flux of heat into the thermocline.

\subsubsection{Hydrographic mixed layers}

Conventional definitions of the mixed layer depth, $H_{\rho}$, reflect variability of regional water mass properties, seasonality, and local mixing processes such as convection. Spatial and temporal patterns of $H_{\rho}$ can be estimated from data from profiling floats 
(Holte and Roemmich (2010), Dong et al. (2008)). The seasonal cycle of the OML includes deep mixed layers $(>250 \mathrm{~m})$ in Austral winter (Jul-Sep) and shallower layers $(<150 \mathrm{~m})$ in Austral summer and fall (Jan-Jun) (Fig. 4-1). In Austral spring (OctDec) the water column restratifies as seasonal heat fluxes begin to weaken. While deep winter mixed layers are known to be driven by seasonal cooling, summer mixed layers in the Southern Ocean are shallow and influenced by local wind forcing events.

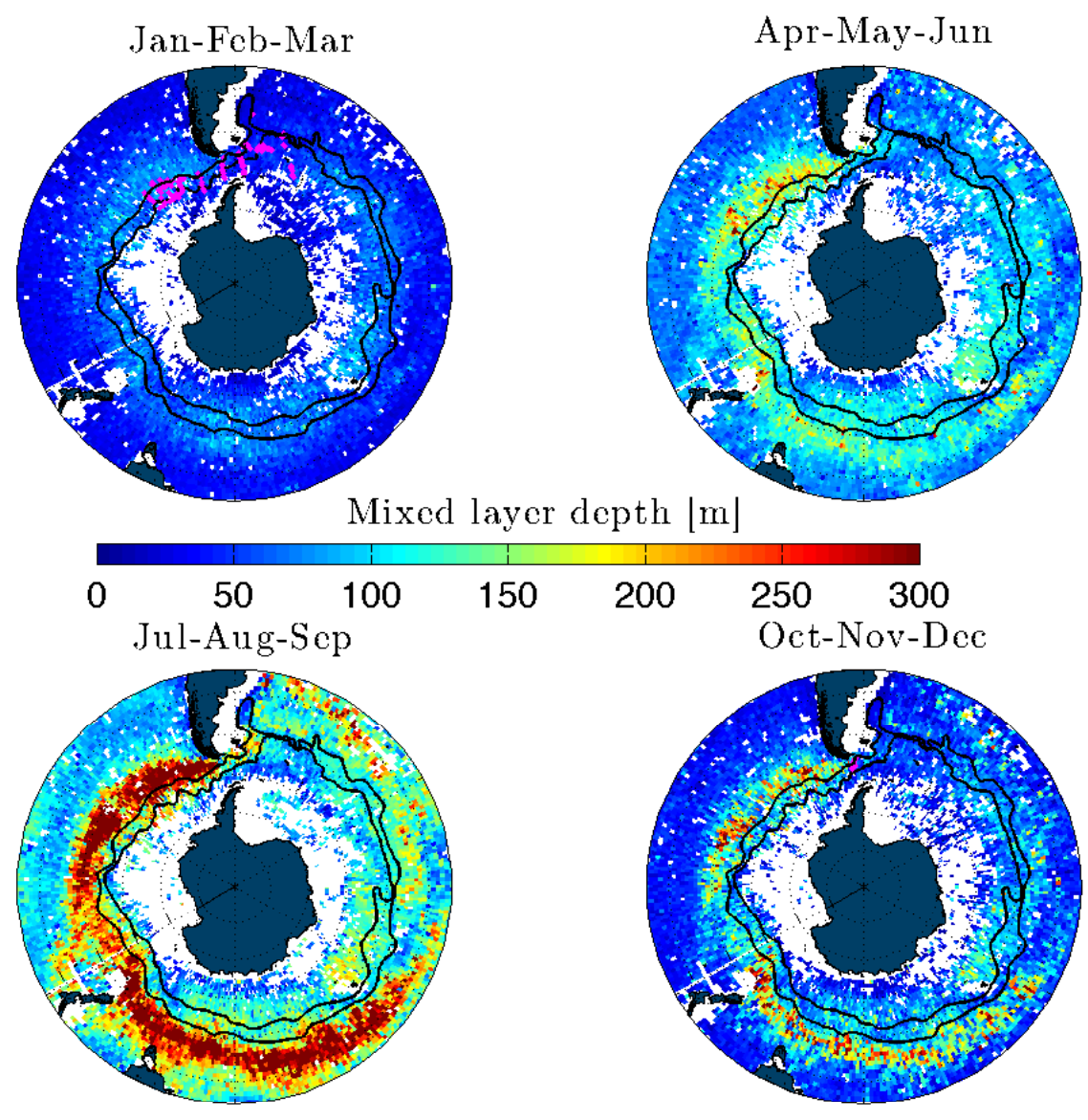

Figure 4-1: Seasonal mean mixed layer depth from the 2010 climatology (Holte and Roemmich (2010)). Pink dots represent the location of DIMES microstructure measurements. All All DIMES data except US5 (Oct-Nov) were collected in Austral Summer (January-March).

The majority of the DIMES data were collected during Austral Summer when the average hydrographic mixed layer depth, $H_{\rho}$, is $50 \mathrm{~m}$ in the region (Fig. 4-1). A strong thermocline extending approximately 50m below the mixed layer (Fig. 4-2) is evident below the well-mixed layers. In Austral Spring, the seasonal thermocline and mixed 
layer erodes such that the upper layer is weakly stratified up to the surface.
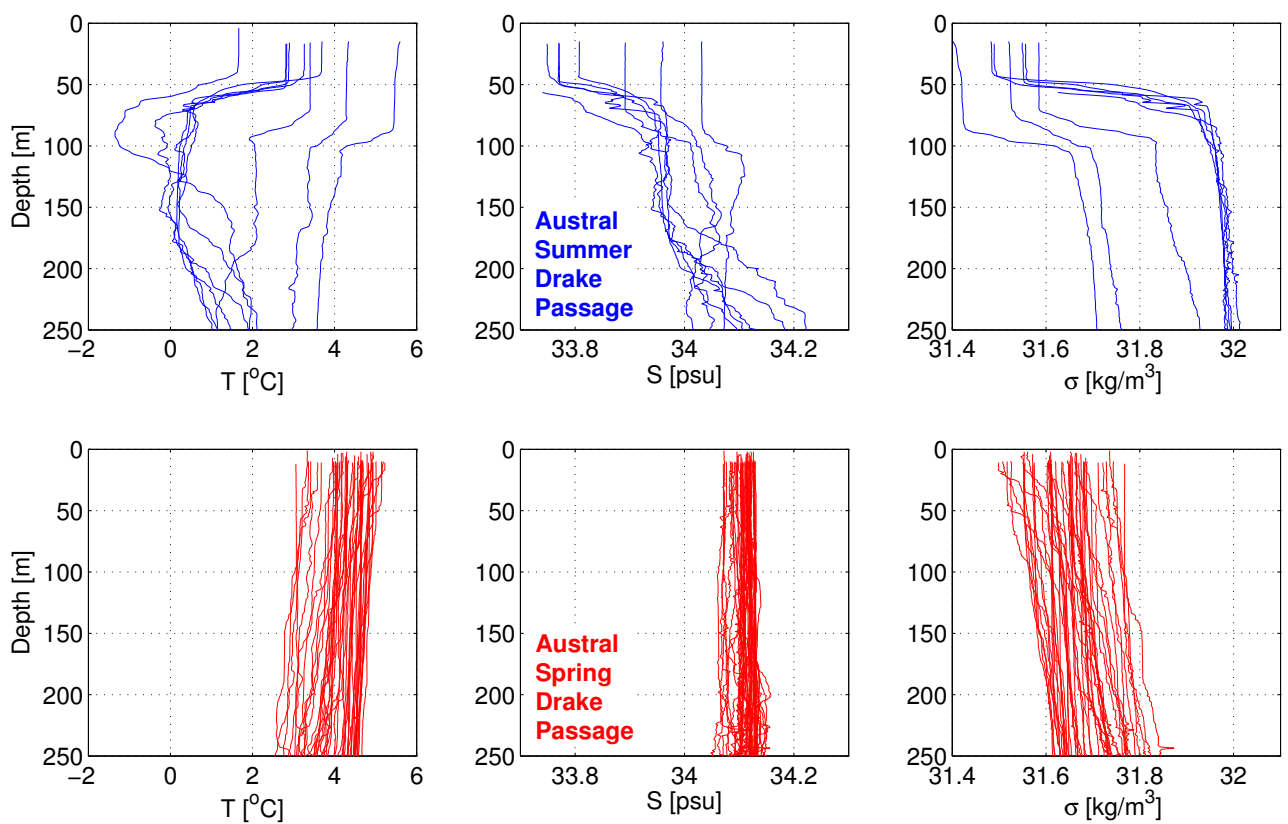

Figure 4-2: Structure of temperature, salinity and potential density as a function of season in Drake Passage. The US2 survey (blue) extended further south in Drake Passage relative to the US5 survey (Red) which was localized in the northern region.

Measurements of vertical velocity shear (Fig. 4-3b), buoyancy frequency (Fig. 4-3c), and dissipation (Fig. 4-3c) during Austral Spring (US5) show the evolution of the OML in time. Our observations reveal a shallow mixed layer of depth $H_{\rho} \approx 65 \mathrm{~m}$ maintained by the strong, predominately zonal wind field. During periods of weaker wind forcing, for example, beginning on Feb. 15, 2010, the OML restratifies near the surface. The seasonal thermocline at $65 \mathrm{~m}$ is ubiquitous throughout the US5 measurements. Enhanced dissipation is evident within the mixed layer with surface enhancement likely associated with the sustained wave field. The energy required to deepen the boundary layer is significant in the presence of the seasonal thermocline.

In Austral spring, the water column restratifies following the erosion of the seasonal thermocline during Austral winter. During a storm with peak winds exceeding 60 knots, the R/V Nathaniel Palmer (US5 expedition) took shelter near Cape Horn and obtained high-resolution tow-yo sections of hydrography and turbulence (Fig. 4-4). Five sections were taken over several hours. The upper panels of Fig. 4-4 show the temperature structure with depth indicating a mixed layer in the upper $50-100 \mathrm{~m}$ of the water column. The lower panels of Fig. 4-4 show that this layer is actively mixing 

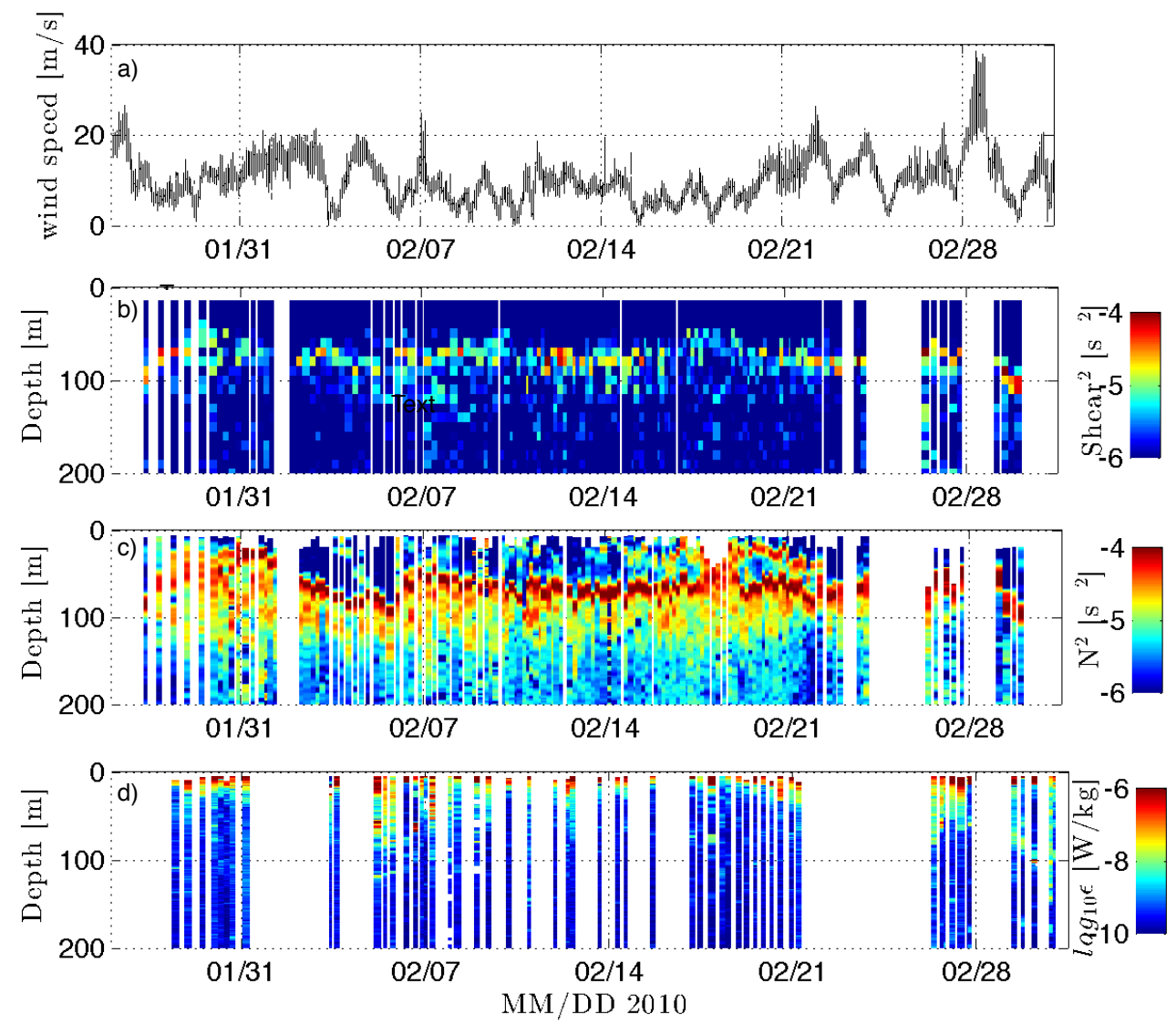

Figure 4-3: The wind speed (a) as a function of time during the US2 deployment coincident to Shear (b), buoyancy frequency (c), and dissipation (d). The seasonal stratification at $75-150 \mathrm{~m}$ exceeds the shear acting to suppress turbulence at the base of the mixed layer.

with values of $\epsilon$ decaying to background values as the stratification increases. The average shipboard wind speed during these measurements was 30 knots. The most enhanced values of dissipation at the surface likely are associated with surface wave physics such as breaking waves or Langmuir turbulence. Variability in the value and structure of $\epsilon$ on hourly timescales and $\mathcal{O}(100 \mathrm{~m})$ spatial scales is a characteristic of submesoscale activity.

The near-surface estimates of turbulent dissipation are consistently large, often exceeding $10^{-6} \mathrm{Wm}^{-2}$. Since the profiler achieved free-fall only after $10 \mathrm{~m}$ depth, all measurements shallower than $10 \mathrm{~m}$ are excluded from the present analysis. Surface wave processes likely are active in the near-surface layer influencing depths up to 

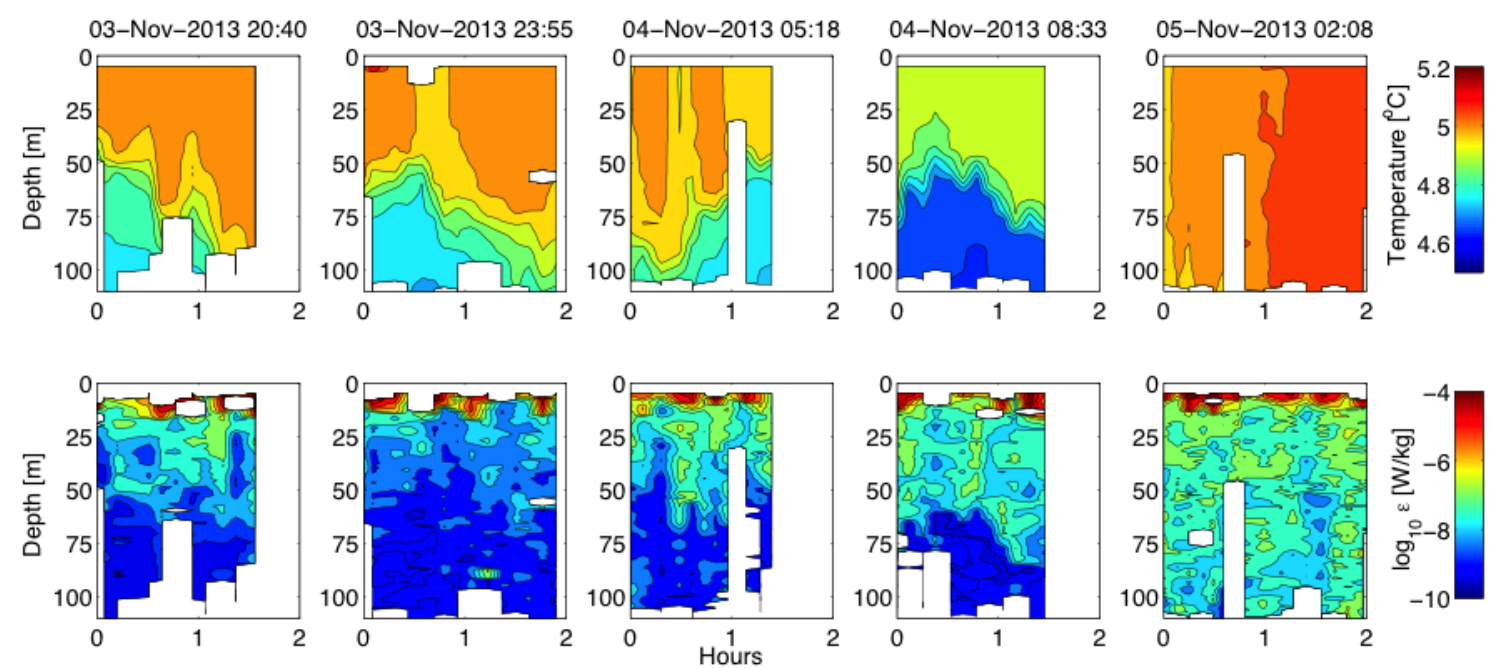

Figure 4-4: Structure of temperature (upper row) and TKE dissipation rate (lower row) for the 5 VMP yo-yo sections taken during a storm. High values of TKE dissipation rate show the same structure as temperature suggesting an actively mixing layer. The very near surface shows large values of $\epsilon$ most likely associated with breaking surface waves.

three times the significant wave height. Below $20 \mathrm{~m}$, the values of dissipation exceeded background values $\left(10^{-9} \mathrm{Wkg}^{-1}\right)$ in the deepening isothermal layer. The tow-yo measurements provide high resolution data in the OML, and are unique in the DIMES project. As these measurements were obtained in Austral spring, active mixing was observed in the weakly stratified near-surface layer due to local wind forcing. The winds decreased to less than 5 knots during the second leg of the tow-yo and observations reveal that the dissipation rates decayed to background layers in the isothermal layer (Fig. 4-4).

\subsubsection{Storm case studies}

The US5 cruise was the only DIMES expedition with both shipboard wind and atmospheric flux measurements. We therefore consider the high-frequency response to storm forcing during two case studies from this expedition. These data allow a full picture of the convective and mechanical forcing of the upper ocean. The average wind speed during the US5 survey exceeded 25 knots, characteristic of the storm track region in Drake Passage. Two strong forcing events occured during the field experiment, one between Nov. 2-5 and the second between Nov. 7-10. During these events, the wind stress reached $1.2 \mathrm{Nm}^{-2}$ (Fig. 4-5a) and turbulent (latent + sensible) 
heat losses exceeded $-300 \mathrm{Wm}^{-2}$ (Fig. 4-5b), where the sign convention is such that positive fluxes represent energy gained by the ocean from the atmosphere. The turbulent heat losses were due to cold polar air that advected into Drake Passage during the storm events. The shortwave and longwave fluxes had daily means of $141 \mathrm{Wm}^{-2}$ and $-23 \mathrm{Wm}^{-2}$, respectively (Fig. 4-5c). The total heat flux (Fig. 4-5d) ranged from a maximum of $650 \mathrm{Wm}^{-2}$ during daylight to $-250 \mathrm{Wm}^{-2}$ at night, with the largest negative values during high storm activity. The largest buoyancy losses occured during the storm events, exceeding $0.8 \times 10^{-8} \mathrm{~m}^{2} \mathrm{~s}^{-3}$ (Fig. 4-5e). The Obukhov length scale, $L$, typically was greater than 100 , such that $\zeta<<1$ suggesting that momentum fluxes due to wind forcing drove the dissipation rates in the boundary layer. During the second storm, values of $L$ approached 10 such that $\zeta \sim 1$ in the boundary layer. This suggests both winds and convective heat loss contributed to the observed values of dissipation.

The Nov. 2-5 storm was driven by a low-pressure system that advected cold air northward (Fig. 4-6). Strong, eastwardly directed winds on the northern side of the cyclone persisted for 3 days. The propagation speed of this system was approximately $7 \mathrm{~m} / \mathrm{s}$. On Nov. 5, the system merged with another low-pressure system downstream. After the storm passed, a high pressure system meandered southward, bringing polar air northward on Nov. 7-8 (Fig 4-6e,f). This advected air was significantly colder than the local SST causing the significant turbulent heat losses between Nov. 7-10.

The strong turbulent heat fluxes beginning Nov. 7, 2015 produced a well mixed layer that deepened at an average rate of $7.5 \mathrm{~m} / \mathrm{hr}$ over 18 hours (Fig. 4-7). The layer was actively mixing until 12PM on Nov. 9, 2015 when the heat flux changed sign. A surface diurnal layer, $0.32^{\circ} \mathrm{C}$ warmer than the well mixed layer developed and extended to $32 \mathrm{~m}$ depth. The heat content in the upper layer is estimated as $\int_{0}^{z_{c}} c_{p} \rho \Delta T d z=4.1 \times 10^{7} \mathrm{~J} / \mathrm{m}^{2}$ where $z_{c}$ is the depth of the capping layer, $c_{p}$ is the heat capacity of sea water at the surface, $\rho$ is the sea water density and $\Delta T$ is the change in temperature from the capping layer to the well mixed layer. The final temperature profile in Fig. 4-7 was taken at the end of the positive phase of the diurnal cycle of heatflux. The integrated net heat flux in Fig. 4-5d over the positive phase amounts to a heat input of $1 \times 10^{7} \mathrm{~J} / \mathrm{m}^{2}$ on Nov. 9. This general agreement between the heat content in the upper layer and the integrated heat flux into the ocean shows that the OML responded to solar forcing via restratification on diurnal timescales. 

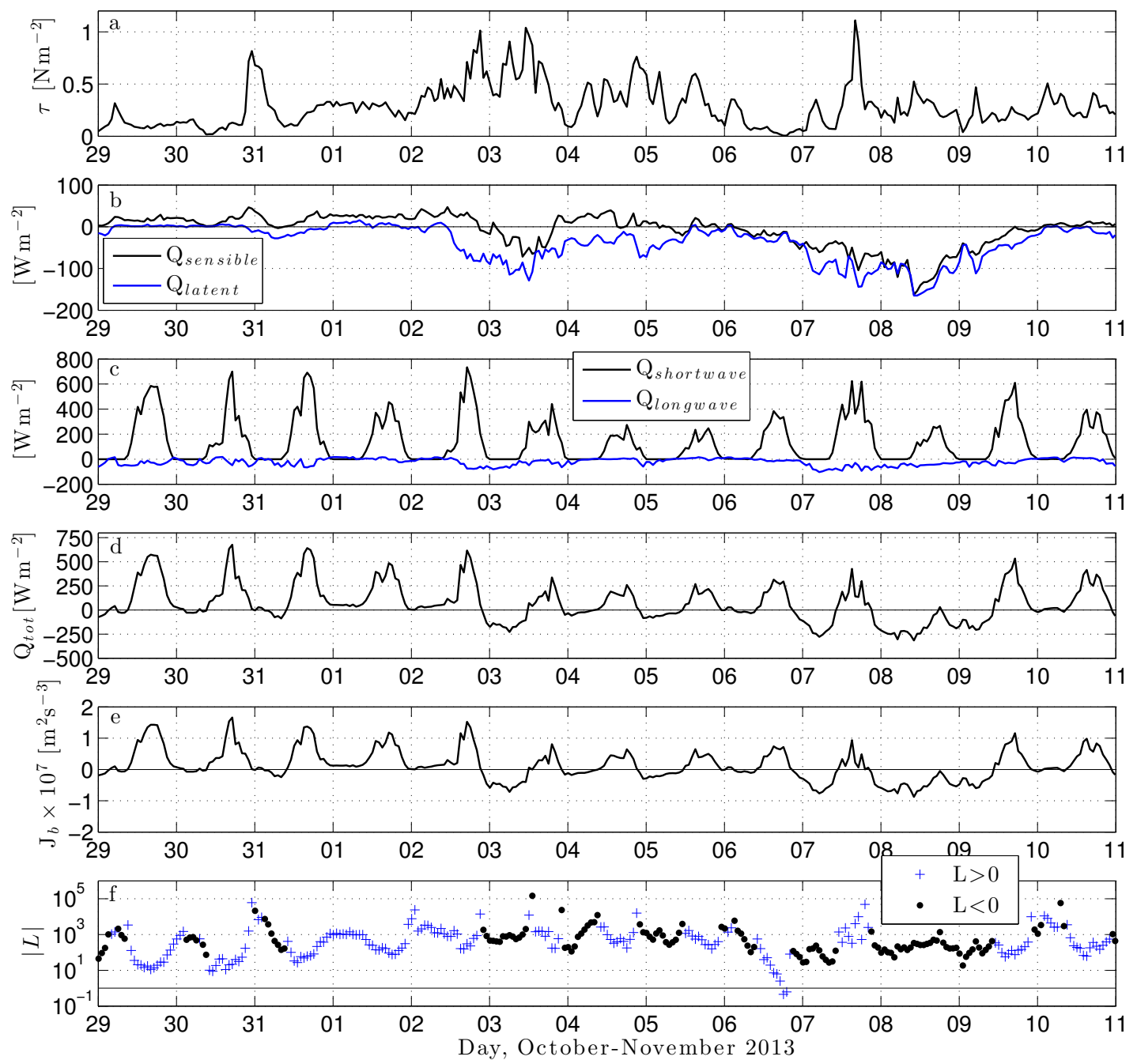

Figure 4-5: The a) wind stress b) latent and sensible heat fluxes c) shortwave and longwave heat fluxes d) total heat flux e) buoyancy flux and f) Monin-Obukhov length during the US5 field program. 

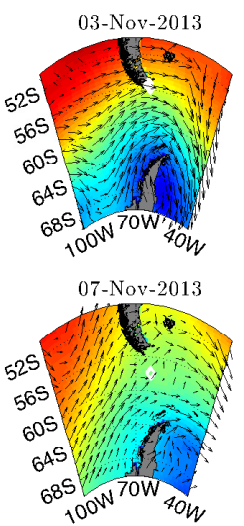
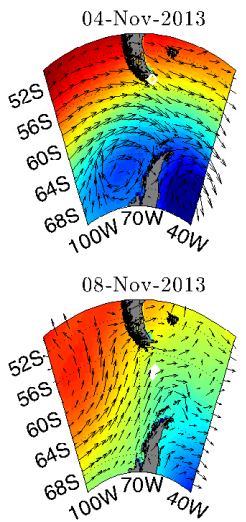
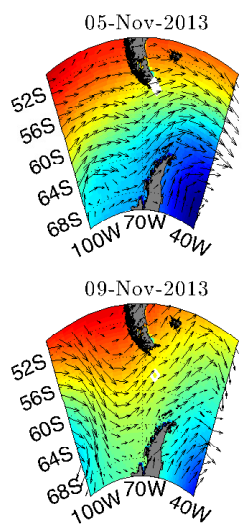
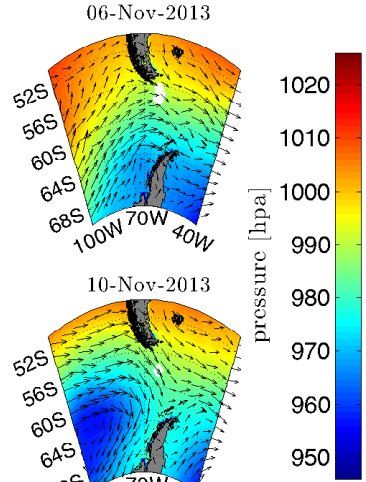

Figure 4-6: Near surface pressure from NASA MERRA during the US5 expedition.

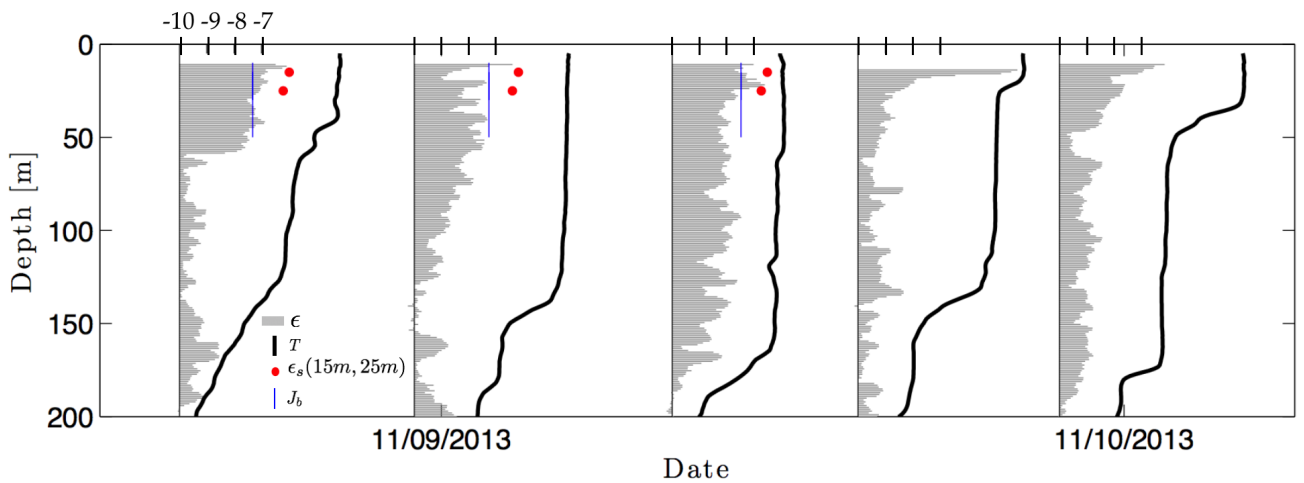

Figure 4-7: Measurements of dissipation (gray) and temperature (black) offset to show the structure of the boundary layer during the second storm (beginning on Nov. 6). The boundary layer deepened in the first three profiles before a capping layer developed as the heat flux changed to stabilizing. The red dots represent values of $\epsilon_{s}$, the Monin-Obukhov scaling in the wind dominated regime. The blue line shows the value of the buoyancy flux, $J_{b}$, during the destabilizing phase.

\subsection{Boundary layer scalings}

We have shown using direct measurements that the OML evolves on diurnal, inertial, and seasonal timescales responding to both mechanical and convective forcing. Solar heating may cap the surface layer while sustained zonal winds and storm events maintain a well-mixed layer during the majority of the dataset. The structure of dissipation in the boundary layer often is parameterized in terms of the surface wind stress and buoyancy forcing. Here we test how well (4.3-4.5) represent the observations of turbulent dissipation in the mixed layer. 

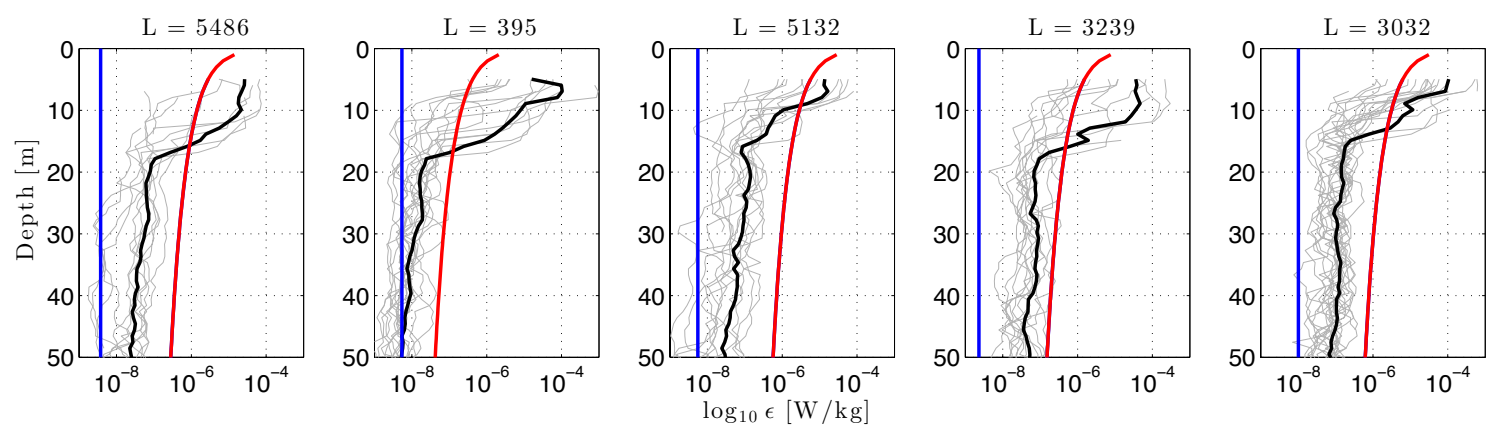

Figure 4-8: Depth structure of measured dissipation from 5 tow-yo transects during US5. The black line represents the transect mean, while the red and blue are the Monin-Obukhov scalings of wind stress, $\epsilon_{s}$ (red), and buoyancy flux, $J_{b}$ (blue). The values of the Obukhov length are given in the title, showing that $\zeta<<1$ throughout the the measurements. This suggests wind-stress dominates turbulent production.

To test (4.3-4.5), we use the 5 tow-yo transects from US5. These data give the highest temporal and spatial resolution and are coincident with a complete set of meteorological measurements from the ship. The tow-yo transects ranged from 1.5-2 hours covering $4.5-7 \mathrm{~km}$. The average profile from each transect is compared to the surface scaling of wind shear and buoyancy forcing (4.3-4.5). Only data collected when the buoyancy flux is destabilizing and shallower than the observed $H_{\rho}$ are used in this analysis. All 5 transects were in the regime where wind-stress dominates turbulence production (Fig. 4-8). A comparison of the observed values of $\epsilon$ to the scalings for wind shear, $\epsilon_{s}$, show that values of $\epsilon$ in the upper $15 \mathrm{~m}$ exceed the scaling. This result is consistent with observations of Agrawal et al. (1992), Drennan et al. (1996) and Terray et al. (1996) who quantified the energy loss from surface waves due to breaking. They found that while the observed value of $\epsilon$ is much larger than $\epsilon_{s}$ due to breaking waves, the $\epsilon_{s}$ scaling becomes valid a few significant wave heights below the surface.

The classical scalings should only hold in the mixed layer, $H_{\rho}$. We estimate the transect averaged $\epsilon / \epsilon_{s}$ when $H_{\rho} /-L<1$ (Fig. 4-9a). This method replicates the study by Lombardo and Gregg (1989) which was used to derive the mixed scaling (4.5). The similarity scaling normalizes the structure of dissipation, however, the average magnitude of $\epsilon / \epsilon_{s}$ is 0.1 . This result suggests that there is less measured dissipation than the wind stress scaling would imply below the wave-driven layer. When the winds weakened, a reduction of turbulent dissipation was observed in the boundary layer. An average of this transect normalized by the buoyancy frequency $\left(\epsilon / J_{b}\right)$ is close to 1 below the wave-breaking layer (10-15m, Fig. 4-9b). This suggests that 

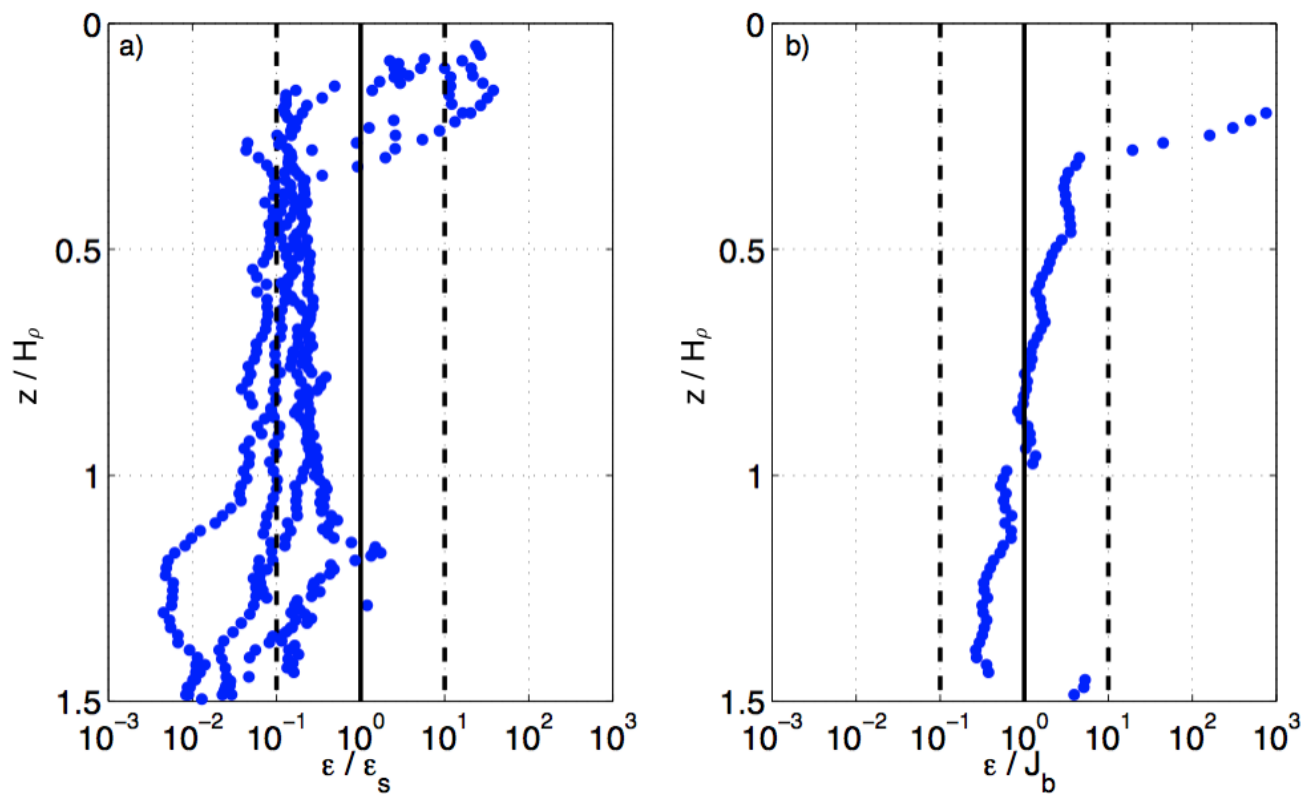

Figure 4-9: Vertical structure of scaled dissipation as a function of depth normalized by the mixed layer depth. Averages are taken along each transect. Figure follows Lombardo and Gregg (1989) Fig. 11.

the convective scaling captures the structure and magnitude of turbulent dissipation under conditions where the winds are weak.

This study shows that the similarity scaling (4.3-4.5) fails in two ways during the measurements collected during DIMES. First, in the surface layer where wave-breaking may be dominant, the scaling underestimates observed values of dissipation. Enhanced dissipation typically is observed in the upper $10-15 \mathrm{~m}$ of the water column. The depth of this layer likely is related to the significant wave height during each measurement (which is supported by a qualitative description of the wave-field) and is consistent with previous observational campaigns (e.g. Terray et al. (1996)). Secondly, the scaling based on the surface wind stress overestimates the magnitude of the observed dissipation in the boundary layer. This scaling has been evaluated using measurements in a weaker wind regime by Lombardo and Gregg (1989). In the extreme wind regime of the Southern Ocean, the surface wave-field may affect transfer of momentum from the wind field to the OML, and this remains an active area of research in both observational and numerical modeling studies (e.g. Drennan et al. (1999), García-Nava et al. (2012), Nilsson et al. (2012)). 


\subsection{Discussion}

DIMES measurements are analyzed to describe the structure and evolution of the OML in the context of diurnal, inertial (storm), and seasonal forcing. The Southern Ocean is unique in that powerful westerly winds energize a strong surface wave field. Conventional scalings based on surface wind and buoyancy forcing do not appear to accurately capture the structure and magnitude of turbulent dissipation in the OML under these extreme conditions. This study is limited by small statistics, lack of wave measurements and meteorological variables measured only during fair-weather conditions. In spite of these limitations, our analysis lends insight into the vertical structure of turbulence in the boundary layer in the Southern Ocean.

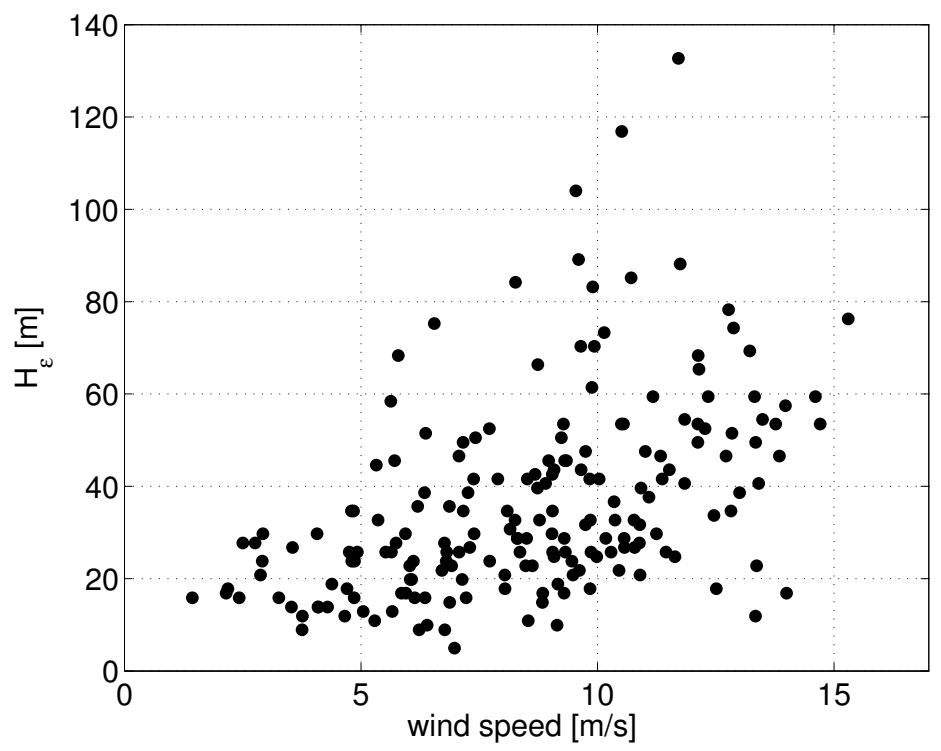

Figure 4-10: The wind speed averaged over 2 hours prior to each microstructure measurement is shown relative to the mixing depth, $H_{\epsilon}$, calculated as the depth where $\epsilon$ decays to $10^{-9} \mathrm{~W} / \mathrm{kg}$.

Our observations suggest that wind stress is generally dominant relative to convection in driving boundary layer turbulence during US5. The Southern Ocean is unique as mean zonal winds energize the surface ocean throughout the year. Fig. 4-10 presents the relationship between the wind forcing and the active mixing layer, $H_{\epsilon}$, estimated from 5 DIMES cruises (described in Table 1). While the active mixing layer depth, $H_{\epsilon}$, generally increases with increasing wind speed, considerable scatter exists suggesting that the state of the surface wave field affects the dissipation of TKE in the mixed layer. 
We further compare the relationship between the mixing depth, $H_{\epsilon}$ and the mixed layer depth, $H_{\rho}$ as a function of wind speed (Fig. 4-11). For higher wind speeds (generally greater than $8 \mathrm{~m} / \mathrm{s}), 0.5 H_{\rho}>H_{\epsilon}>H_{\rho}$. For weaker winds, the actively mixing layer depth is less than $50 \%$ of the depth of the mixed layer. Mixing parameterizations that assume that $H_{\rho}=H_{\epsilon}$ (Sutherland et al. (2014)) potentially overestimate boundary layer dissipation rates over a range of wind conditions in the Southern Ocean.

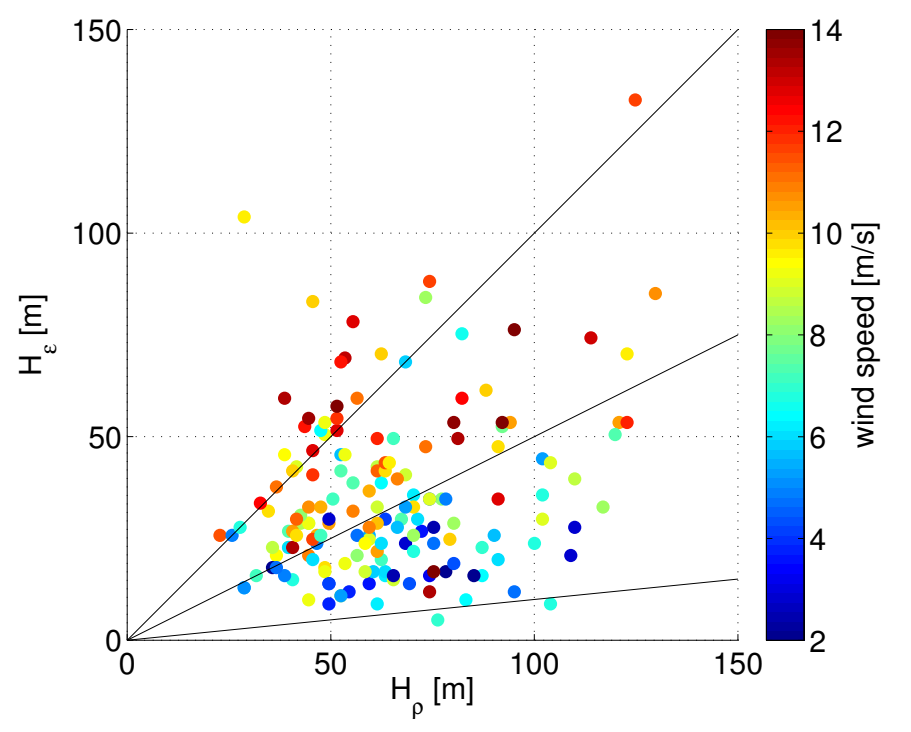

Figure 4-11: Actively mixing layer depth vs. the mixed layer depth where the colors represent the wind speed averaged 2 hours prior to the measurement of turbulence. The lines represent (from top to bottom), $H_{\epsilon} / H_{\rho}=1, H_{\epsilon} / H_{\rho}=0.5$ and $H_{\epsilon} / H_{\rho}=0.1$.

Beyond the classical scalings, the importance of surface waves and frontal instabilities in setting the magnitude of turbulent mixing in the OML is poorly understood in the Southern Ocean. The potential role of Langmuir turbulence and submesoscale instabilities in our study area is discussed below. While adequate data is not available to conclusively address the effects of Langmuir turbulence and submesoscale instabilites, the current results motivate the design of a future observational study and are included here for completeness. 


\subsubsection{Langmuir turbulence}

Langmuir turbulence is characterized in terms of the turbulent Langmuir number,

$$
L a=\sqrt{\frac{u^{*}}{U_{\text {stokes }}}}
$$

where $u^{*}$ is the surface friction velocity and $U_{\text {Stokes }}$ is the surface Stokes drift velocity. The Langmuir number measures the relative importance of wind generated shear (characterized by friction velocity $u^{*}$ ) and the nonlinearity of the wave field that generates the Stokes drift $\left(U_{\text {stokes }}\right)$. The interaction between the two processes results in Langmuir circulation, which may deepen the mixed layer (Craik and Leibovich (1976)). When $L a \leq 0.3$ Langmuir turbulence is believed to dominate shear turbulence. The transition from Langmuir to shear turbulence occurs when $0.5<L a<2$ (Grant and Belcher (2009)).

Strong westerly winds in the Southern Ocean force steep waves and potentially enhanced Langmuir turbulence. Efforts to study Langmuir circulation and turbulence from scatterometer measurements have shown that the Southern Ocean is a region of enhanced winds and Stokes drift (Fig. 4-12a,b, Liu et al. (2014)). The resulting Langmuir number (Fig. 4-12c), is low in the Southern Ocean, indicative of the potential contribution of Langmuir turbulence in the region.

In addition to hydrographic measurements, novel DIMES upper ocean turbulence measurements provide insights into boundary layer parameterizations in the OML. Observations reveal that TKE dissipation is enhanced in the upper 15-20m suggesting that surface wave breaking is active over depths up to three times the significant wave height. The conventional Monin-Obukhov scalings used in many boundary layer schemes underestimate the magnitude of turbulence dissipation during US5 below this active surface layer. The breakdown of (4.3-4.5) during storm conditions suggests that the surface wave field may play an important role in the OML dynamics in this region. Developing a better understanding of the effects of the surface wave field on the OML is a topic of future study. Finally, observations suggest that additional processes maybe responsible for dissipating energy in the boundary layer. The Southern Ocean is an obvious candidate for Langmuir turbulence and submesoscale instabilities: the high winds suggest the potential for Langmuir turbulence (Figure 4-12), and

conditions for frontal instabilites are favorable in this region (Appendix 4.5). Our preliminary observational study highlights the need for additional measurements, in 

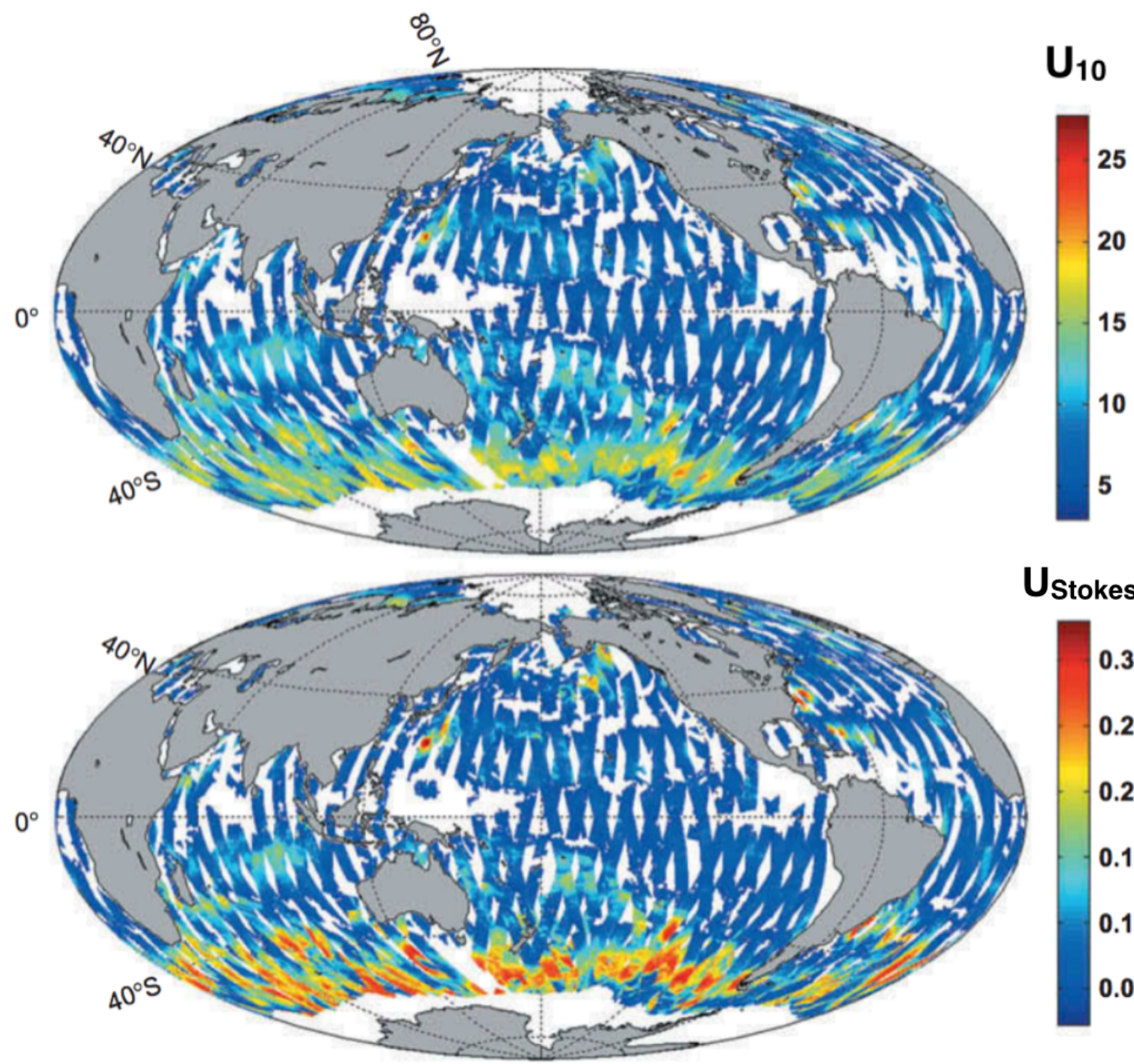

\section{Ustokes}
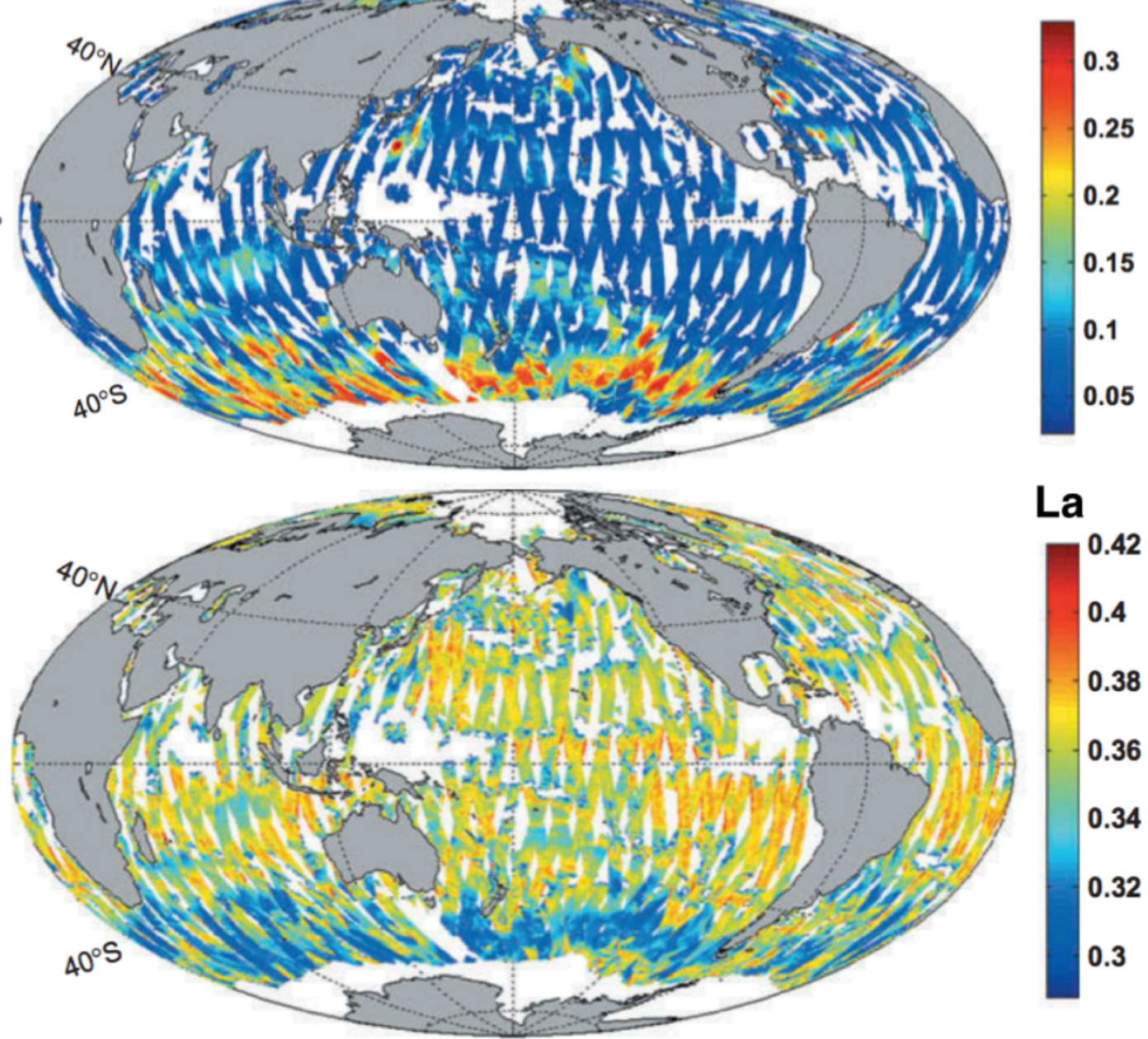

Figure 4-12: a) Strong wind speed in the Southern Ocean, b) Stokes drift, and as a result the Langmuir number, $L a$ is often lower than 0.3 in the Southern Ocean. Figures adapted from Liu et al. (2014) 
particular wave information, in this region of the ocean. Nonetheless, the DIMES experiment has provided the most comprehensive microstructure measurements in this region to date.

\subsection{Appendix}

\subsubsection{Submesoscale instabilities}

While mixing in the surface boundary layer of the ocean typically is considered a one-dimensional process, the presence of lateral density gradients on scales of 1-10km highlight the three-dimensional nature of processes that may lead to turbulence and mixing. In addition to wave-driven processes in the boundary layer, the Southern Ocean is characterized by fronts which form as mesoscale eddies interact with the large-scale density field. Frontal regions are often the location of mixed layer instabilities (Boccaletti et al. (2007)) which may shoal the wintertime mixed layer (Fox-Kemper et al. (2011)). Submesoscale instabilities provide a means of dissipating kinetic energy in the ocean circulation. Thomas et al. (2013) investigated the role of symmetric instability (SI), a class of submesoscale instability, in removing energy from geostrophic flows in the Gulf Stream, a current with similarities to the ACC.

Here, we study the submesoscale density field using the Ertel potential vorticity (PV), which has been used to diagnose instabilities in the surface boundary layer (Hoskins (1974)). In a Cartesian system, the Ertel PV has the form:

$$
Q=-\frac{\left(2 \vec{\Omega}+\zeta_{r}\right) \cdot \nabla \rho_{\theta}}{\rho}
$$

where $2 \vec{\Omega}$ represents the earth's axis of rotation, $\zeta_{r}$ is the relative vorticity, $\rho$ is the density, and $\rho_{\theta}$ is the potential density.

The potential vorticity, $Q=Q_{v}+Q_{b c}$ has contributions from the vertical component of the absolute vorticity

$$
Q_{v}=N^{2}\left(f-\frac{\partial u}{\partial y}+\frac{\partial v}{\partial x}\right)
$$


and the horizontal component along the horizontal gradient of buoyancy:

$$
Q_{b c}=\left(\frac{\partial u}{\partial z}-\frac{\partial w}{\partial x}\right) \frac{\partial b}{\partial y}+\left(\frac{\partial w}{\partial y}-\frac{\partial v}{\partial z}\right) \frac{\partial b}{\partial x}
$$

To first order, the system is assumed to be in thermal wind balance such that:

$$
Q_{b c}=-f\left(\frac{\partial u_{g}}{\partial z}\right)^{2}=\frac{g}{f \rho_{0}}\left|\nabla_{h} \rho\right|^{2}
$$

The overturning instabilities develop when $\mathrm{Q}$ opposes the sign of $f$, hence in the Southern Hemisphere, instabilities will occur in those regions where $\mathrm{Q}$ is positive. Inertial instability occurs in barotropic flows when $Q>0, Q_{v}>0$, and $Q_{b c}=0$. When $Q>0$ and $Q_{b c}>Q_{v}$, the instability is known as symmetric instability (SI). SI occurs in frontal regions that are characterized by large vertical shear and strong horizontal density gradients. In the ACC where the submesoscale frontal field is rich, symmetric instability can reduce the wind-work available to the geostrophic circulation (Thomas and Taylor (2010)). In this sense kinetic energy transferred to the large scale circulation by the wind is reduced through a submesocale sink of kinetic energy through shear instabilities.

Building on experiments in the Kuroshio (D'Asaro et al. (2011)) and the Gulf Stream (Thomas et al. (2013), Todd et al. (2016)), two boundary currents with many similar properties to the ACC, the rich submesoscale frontal field provides a link between mesoscale and dissipation scale motions, and can cascade energy to smaller scales. These $\mathrm{O}(1-10 \mathrm{~km})$ fronts provide an energy source for enhanced turbulent dissipation in the upper ocean when released by downfront winds. Further data in this region will allow us to test the hypothesis that small-scale overturning instabilities provide the energy to large dissipation rates in the upper ocean. 


\section{Chapter 5}

\section{Near-inertial energy and turbulence in the wake of storms}

\section{$5.1 \quad$ Introduction}

A number of studies have suggested that mixing rates in the upper $1.5 \mathrm{~km}$ of the Southern Ocean are influenced by breaking near-inertial internal waves (NIW) generated in the wake of storms (e.g. Wu et al. (2011), Thompson et al. (2007)). These studies have used indirect methods to estimate mixing rates from finescale hydrographic and velocity measurements. In situ microstructure measurements collected in the Southern Ocean as part of DIMES yield mixing rates significantly weaker than the reported parameterized rates (e.g. Frants et al. (2013), Merrifield et al. (2016)). This discrepancy raises a number of issues regarding the nature of finestructure variability and the pathways to mixing. In particular, the importance of NIWs for upper ocean mixing has not been well defined, including open questions regarding the efficiency of wind work on NIWs by Southern Ocean storms, the extent to which NIW energy passes from the surface mixed layer to the stratified interior, and the reasons why finescale-based mixing estimates, which presumably are strongly influenced by the presence of NIW energy, apparently over-estimate mixing rates.

To address these issues, in situ upper ocean observations are needed in the Southern Ocean during energetic storms; however, prior to DIMES few datasets were available. Shipboard measurements suffer from a fair weather bias making it difficult to quantify 
the energy imparted to the surface ocean during storm events. Moveover, there is a lack of in situ air-sea flux measurements in this region, making it difficult to assess the work done by surface wind stress on the upper ocean. The first moored buoy began recording in 2010, located southwest of Tasmania (Schulz et al. (2012)). Lacking direct observations, estimates of wind work and the generation of NIW energy have been made using wind stress products, often with a temporal resolution of 6 hours (e.g. Alford (2003)). A 6-hour product poorly resolves inertial period motions, which at $60^{\circ} \mathrm{S}$ have a period of approximately 14 hours. Studies that have used coarse temporal wind products have consistently estimated very weak values $\left(<2 \mathrm{mWm}^{-2}\right)$ of energy flux from the winds to near-inertial motions in the Southern Ocean (Alford (2003), Elipot and Gille (2009)). In addition to the poor temporal sampling of inertial motions by atmospheric products, wind work estimates generally rely on a mixed layer model to relate winds with upper layer currents. The slab model of Pollard and Millard (1970) has been used extensively (e.g. D'Asaro (1985), Watanabe and Hibiya (2002)); however, NIW energy flux estimates based on this model are sensitive to uncertainties in mixed layer depth, and to the choice of a damping parameter (Plueddemann and Farrar (2006), Kilbourne and Girton (2015)).

In general, the ability of winds to generate inertial motions plays a significant role in the kinetic energy budget of the upper ocean. The upper ocean response to wind stress forcing can include development of near-inertial internal waves, higher fequency internal waves, and enhanced shear at the base of the mixed layer (Plueddemann and Farrar (2006)). Enhanced shear and wave generation at the base of the mixed layer can lead to enhancement of dissipation both locally, through shear instability, and non locally, as the near-inertial waves propagate in the interior, interact with the background wave field and eventually break.

Estimating the amount of NIW energy that penetrates the thermocline and feeds the interior internal wave field is difficult. Estimates from a study of storms in the tropics suggest that $10 \%$ of near-inertial energy is able to reach the interior (Cuypers et al. (2013)). Large values of dissipation in the near-surface layer can act as a sink of nearinertial energy. For example, Zhai et al. (2009) estimated $70 \%$ of near-inertial energy imparted by the wind is lost to turbulent mixing in the upper 200m. Energy that is able to exit the mixed layer may escape as low-mode near-inertial waves (D'Asaro et al. (1995)). These waves can propagate equatorward where the local $f$ is decreasing, but not poleward. Model simulations by Simmons and Alford (2012) suggest as much as $16 \%$ of near-inertial wave energy radiates long distances equatorward in low modes. 
Near-inertial kinetic energy in mid-latitude studies shows a strong enhancement in winter due to increased storm intensity and frequency (Silverthorne and Toole (2009)). Surprisingly, a seasonal cycle in near-inertial kinetic energy has not been detected in the Southern Ocean, despite energetic storm forcing. Alford and Whitmont (2007) attribute this to a weaker seasonal cycle in the storm forcing.

This study uses a variety of in-situ measurements to study the near-inertial and turbulent response of the ocean to strong wind forcing in the Drake Passage region. To assess the magnitude of the NIW energy flux to the mixed-layer via surface forcing, three methods are used:

1. Surface-drifter derived inertial velocities and high-resolution shipboard wind measurements are used to estimate NIW flux.

2. Slab model forced by the high-resolution ship record.

3. Slab model forced by a 6 -hour reanalysis product.

We compare the three methods in Section 5.2.2. We then address the relationship between turbulence levels and downward radiating near-inertial shear in an effort to study the effects of surface wind forcing on mid-depth mixing rates (5.3).

\subsection{Near-inertial energy flux to mixed layer motions}

The efficiency of surface winds at inputting near-inertial energy to the mixed layer in the Southern Ocean has been difficult to assess due to a lack of in situ measurements. The DIMES study included shipboard meteorological measurements that captured the high-resolution spatial and temporal variability in the Drake Passage region during energetic storm events. Here, we evaluate three different estimates of the near-inertial energy flux input to the ocean due to surface wind forcing using (1) drifter and shipboard wind observations following Silverthorne and Toole (2009), and the slab

model of Pollard and Millard (1970) forced by (2) shipboard winds and (3) wind reanalysis products. 


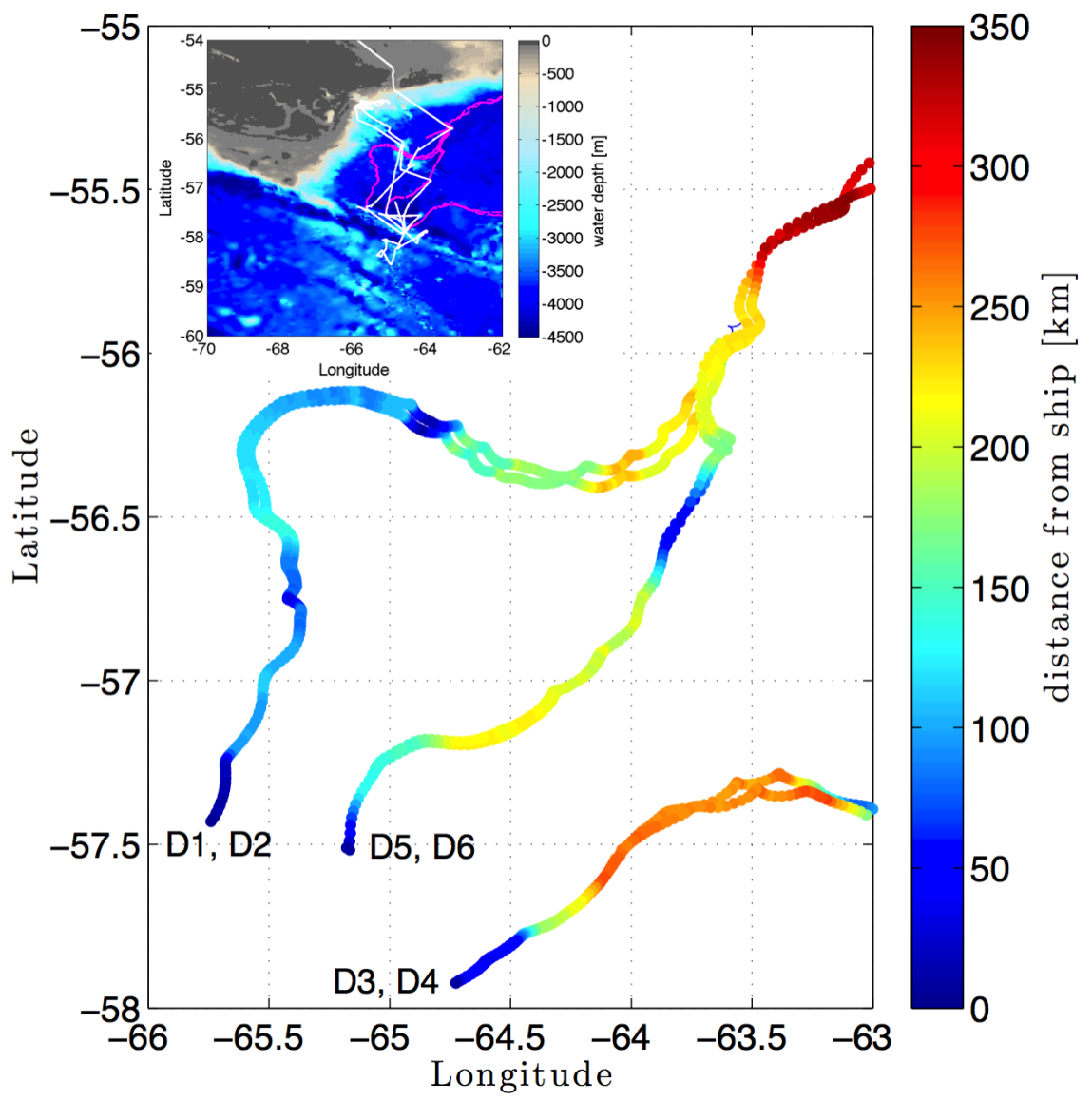

Figure 5-1: Map of the 3 drifter pair trajectories colored by the distance from the ship. Inset is a map with the cruise track (white) and drifter tracks (magenta) overlayed on water depth. 


\subsubsection{Estimate from surface drifters and shipboard winds}

Three pairs of surface drifters with $15 \mathrm{~m}$ drogues were deployed over the course of the DIMES US5 cruise to gather estimates of Lagrangian drift patterns concurrently with shipboard wind measurements. The ship and the drifters were never further than $400 \mathrm{~km}$ apart, well within a typical atmospheric synoptic scale $(\sim 1000 \mathrm{~km}$, Fig. $5-1)$. Each drifter exhibited anticyclonic motions following the onset of energetic wind events that were suggestive of wind-driven inertial motions in the mixed layer.

We isolate inertial currents using a bandpass filter in the frequency range $0.65 f-$ $1.5 f$ (Fig. 5-2a). We consider motions with frequencies as low as $0.65 f$ because inertial motions can occur at frequencies less than $f$ associated with the modified inertial frequency $f_{\text {eff }}=f+\frac{\zeta}{2}$ (Weller (1982)) where $\zeta$ is the relative vorticity of mesoscale motions. The mesoscale eddy field is active as fronts converge and meander through Drake Passage, such that enhanced inertial energy is expected at subinertial frequencies. Semidiurnal barotropic tides fall into the near-inertial frequency band at these latitudes, therefore the surface drifter velocities are de-tided using the TPXO (Egbert et al. (1994)) zonal and meridional velocity predictions at $65^{\circ} \mathrm{W} 57^{\circ} \mathrm{S}$ (Fig.5$2 \mathrm{~b}, \mathrm{c})$. We note that semidiurnal internal tides may remain in the near-inertial band current estimates.

The work done by surface wind stress on mixed layer currents, referred to here as the energy flux from the wind to mixed layer motions, is estimated using wind stress $(\tau)$ based on shipboard wind measurements and drifter currents $(u)$. The instantaneous energy flux is estimated as $E=\vec{\tau} \cdot \vec{u}$ (Fig. 5-3a). The largest E values occurred during strong wind events. Following Silverthorne and Toole (2009), the near-inertial energy flux also is estimated as $E_{i}=\vec{\tau}_{i} \cdot \vec{u}_{i}$, where the wind stress is bandpassed in the same manner as the inertial currents. The fact that $E>>E_{i}$ suggests a majority of the energy flux goes into low frequencies. $E_{i}$ is positive when the wind and inertial currents are in phase, negative when they are $180^{\circ}$ out of phase, and zero when they are in quadrature. $E_{i}$ is estimated for the 6 drifters deployed before Nov. 2 (Fig. $5-3 \mathrm{~b})$. The value of $E_{i}$ averaged over the cruise record is $2.3 \mathrm{mWm}^{-2}$ with a maximum value of $25 \mathrm{mWm}^{-2}$. The largest values occur during Nov. 7 , when a strong, impulsive wind stress was measured by the ship. This result is consistent with the idea that near-inertial energy enters the surface ocean episodically. The majority of the energy flux to the mixed layer is attributed to a few forcing events. 

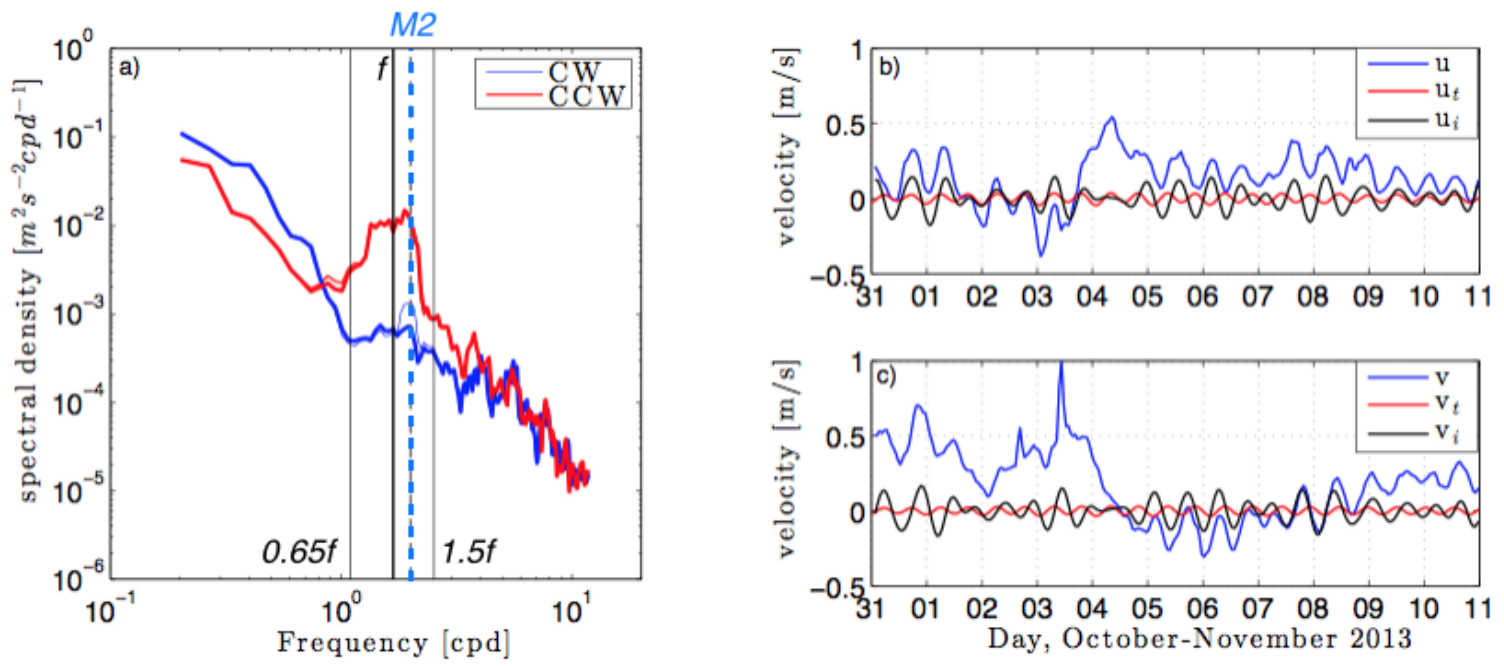

Figure 5-2: a) Rotary spectrum of a representative drifter velocity time series (D1) shows significant CCW energy at near-inertial frequencies. The thick solid vertical line denotes the mean inertial period over the course of the drifter deployment, the dashed line the M2 frequency, and the thin lines the 0.65-1.5f frequency band. The thin blue line is the spectra before de-tiding. b) The zonal current component $(\mathrm{u})$ estimated from drifter D1, compared with the near-inertial bandpass current $\left(u_{i}\right)$ and the TPXO tidal current $\left(u_{t}\right)$. c) Same as (b) for the meridional current component.

\subsubsection{Slab model}

An analytic model of a slab mixed layer response to surface wind stress forcing, developed by Pollard and Millard (1970) and extended by D'Asaro (1985) is used to estimate inertial currents in the mixed layer. The model currents then are combined with the surface wind stress to estimate $E_{i}$, the work done by the wind on the mixed layer at inertial frequencies.

The slab model is based on the $\mathrm{x}$ (zonal) and $\mathrm{y}$ (meridional) momentum equations

$$
\begin{aligned}
& \frac{d u}{d t}-f v=\frac{\tau_{x}}{H \rho}-r u \\
& \frac{d v}{d t}+f u=\frac{\tau_{y}}{H \rho}-r v
\end{aligned}
$$

where $u$ and $v$ are velocity components in the $\mathrm{x}$ and $\mathrm{y}$ directions, $\rho$ is the density, and $\mathrm{H}$ is the mixed layer depth. The components of the windstress, $\tau_{x}$ and $\tau_{y}$, are computed using the relationship $\vec{\tau}=\rho_{a} C_{d} \vec{u}_{w}|\vec{u}|_{w}$ where $C_{d}$ is the neutral drag coefficient estimated following Large and Pond (1981), $\rho_{a}$ is air density and $\vec{u}_{w}$ is the 

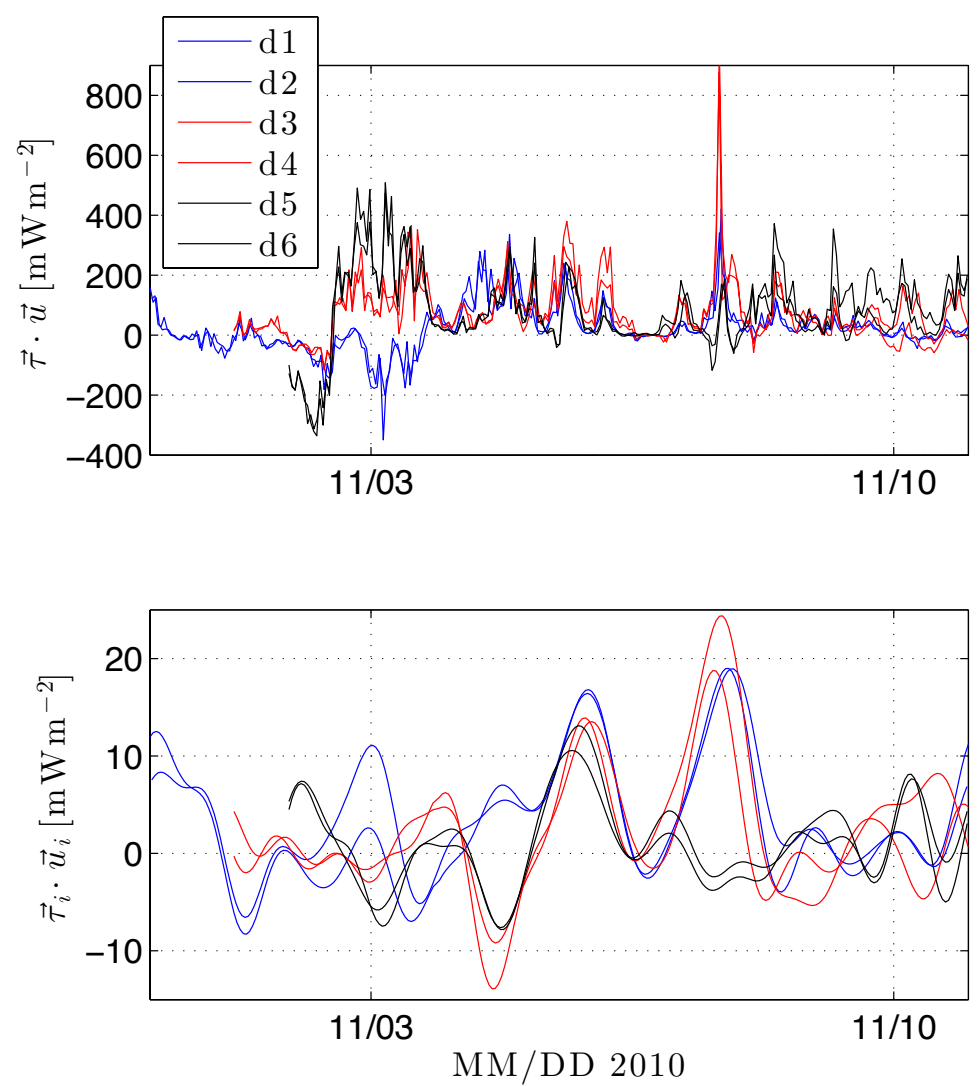

Figure 5-3: The (a) total and (b) inertial energy flux from the wind to mixed layer motions evaluated along the path of each drifter. c) Path of the six drifters with the distance between each drifter and the ship indicated in color. 
wind speed vector. The damping term, $r$, acts to represent the loss of energy in mixed layer motions to internal wave propagation.

Equations 5.1 and 5.2 can be written in terms of the following complex variables

$$
T=\frac{\tau_{x}+i \tau_{y}}{\rho} \quad Z=u+i v \quad \Omega=r+i f
$$

yielding a horizontal momentum equation in terms of $\mathrm{Z}$

$$
\frac{d Z}{d t}+\Omega Z=\frac{T}{H}
$$

The solution to equation 5.3 is:

$$
Z(t)=\frac{\int \exp \left(\int \Omega(t) d t\right) \frac{T}{H} d t}{\exp \left(\int \Omega(t) d t\right)}+C
$$

where $C$ is an unknown constant.

We evaluate equation 5.4 for conditions during the US5 cruise. The integrations are performed using a backward Euler method and a one second timestep. The model is forced by shipboard winds with 5 -minute temporal resolution.

The model results are sensitive to the choice of a linear damping term that parameterizes the transfer of energy from the mixed layer to the deep ocean (Plueddemann and Farrar (2006)). D'Asaro et al. (1995) found good agreement between observations and the slab model for values of $1 / \mathrm{r}$ between 2 and 10 days for an experiment in the northeast Pacific Ocean at $139^{\circ} \mathrm{W} 47.5^{\circ} \mathrm{N}$. Alford (2003) found that the inertial energy flux is insensitive to the magnitude and functional form of $r$ when $r<<f$. There are also well-documented sensitivies of the slab-model to the mixed layer depth $H$ (e.g. Watanabe and Hibiya (2002)). The dataset is collected over a 3 week time period such that seasonal changes in the mixed layer depth can be neglected. For simplicity, we consider a fixed mixed layer depth and damping parameter.

Generally, reanalysis or mooring data are used to provide an Eulerian measurement of surface wind stress which can then be integrated over a few inertial periods as the ocean adjusts. Because the cruise-track data are non-Eulerian, we treat the shipboard data as a local timeseries, with the inherent assumption that the characteristic deformation length scale of the winds is larger than the ship can move over a few inertial periods. The fastest travel speed of the boat was $6 \mathrm{~m} / \mathrm{s}$ such that over an inertial 
period ( $\sim 14$ hours) the ship traveled at most $300 \mathrm{~km}$. Given a typical atmospheric deformation scale of $1000 \mathrm{~km}$, the ship stays within an average synoptic scale system for multiple inertial periods. We note that unresolved variability in the wind field on scales smaller than $300 \mathrm{~km}$ may influence the estimates.

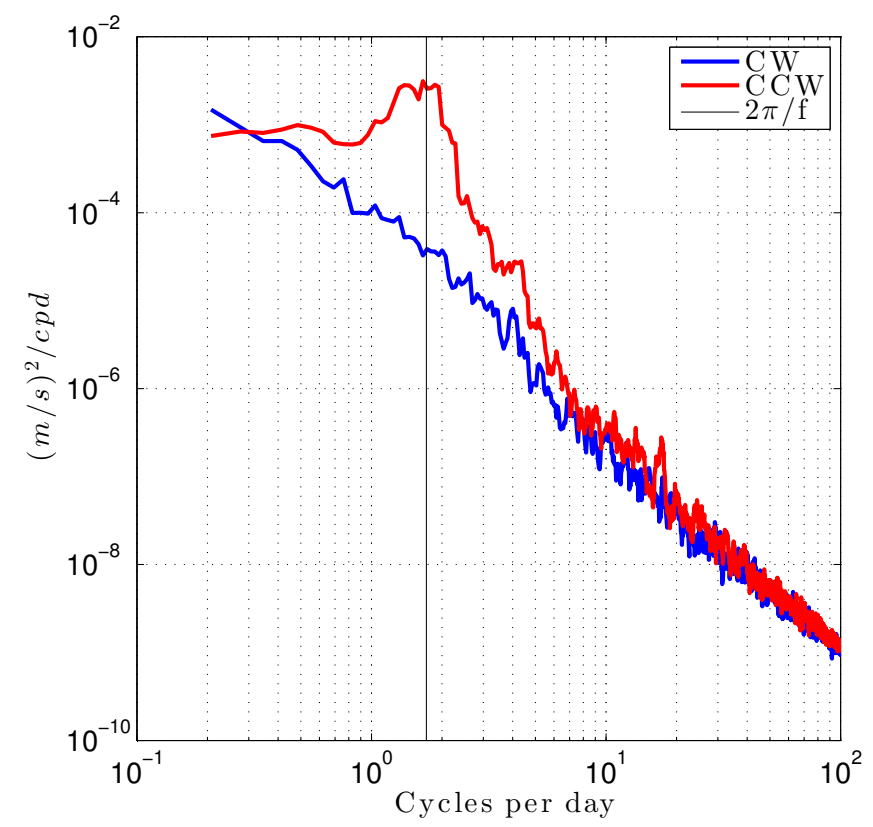

Figure 5-4: Rotary frequency spectra of the velocity estimated from the slab model.

The resulting mixed layer currents have a CCW rotary spectral peak near the inertial frequency that resembles the observed rotary spectrum estimated from drifters (Fig. 5-4). To study the flux of energy from the wind to near-inertial motions, the mixed layer velocity is spectrally filtered to estimate the near-inertial velocity in the mixed layer. The flux, $E_{i}=\vec{\tau}_{i} \cdot \vec{u}_{i}$, is estimated in the same manner as the previous section. Setting $r$ and $H$ to be constants, equation 5.4 becomes:

$$
Z(t)=\frac{1}{H} \frac{\int \exp (\Omega t) T d t}{\exp (\Omega t)}+C
$$

The mixed layer depth is inversely related to the current. We first consider estimates based on $\mathrm{H}=50 \mathrm{~m}$, estimated from the shipboard hydrographic data, and $\mathrm{r}=0.15 \mathrm{f}$ (following Alford (2003)). The resulting mixed layer currents are generally weak, with a standard deviation of $4.5 \mathrm{~cm} / \mathrm{s}$. The near-inertial velocity, estimated with a spectral filter, dominates the variance (Fig. 5-5), with a standard deviation of $3.6 \mathrm{~cm} / \mathrm{s}$. Reducing the damping parameter $(\mathrm{r}=0.02 \mathrm{f})$ to represent weak energy loss 
to the interior results in an amplification of the current, with a standard deviation of $7.7 \mathrm{~cm} / \mathrm{s}$.
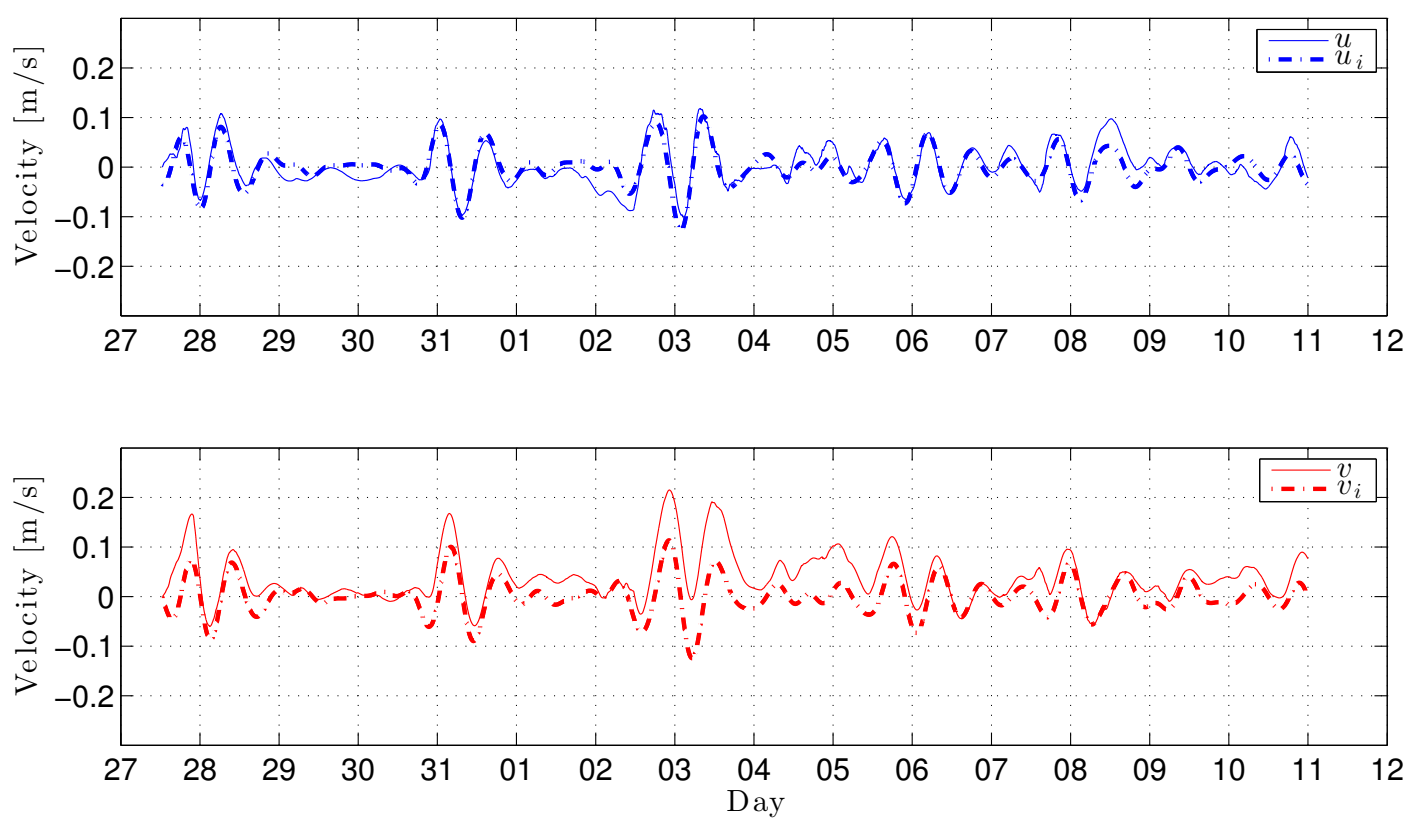

Figure 5-5: a) Zonal and b) meridional velocity (solid lines) estimated from the slab model forced with shipboard winds and a constant mixed layer $H=50 \mathrm{~m}$ and $r=0.15 f$. The dashed line is the bandpass filtered near-inertial current which dominates the variance. 

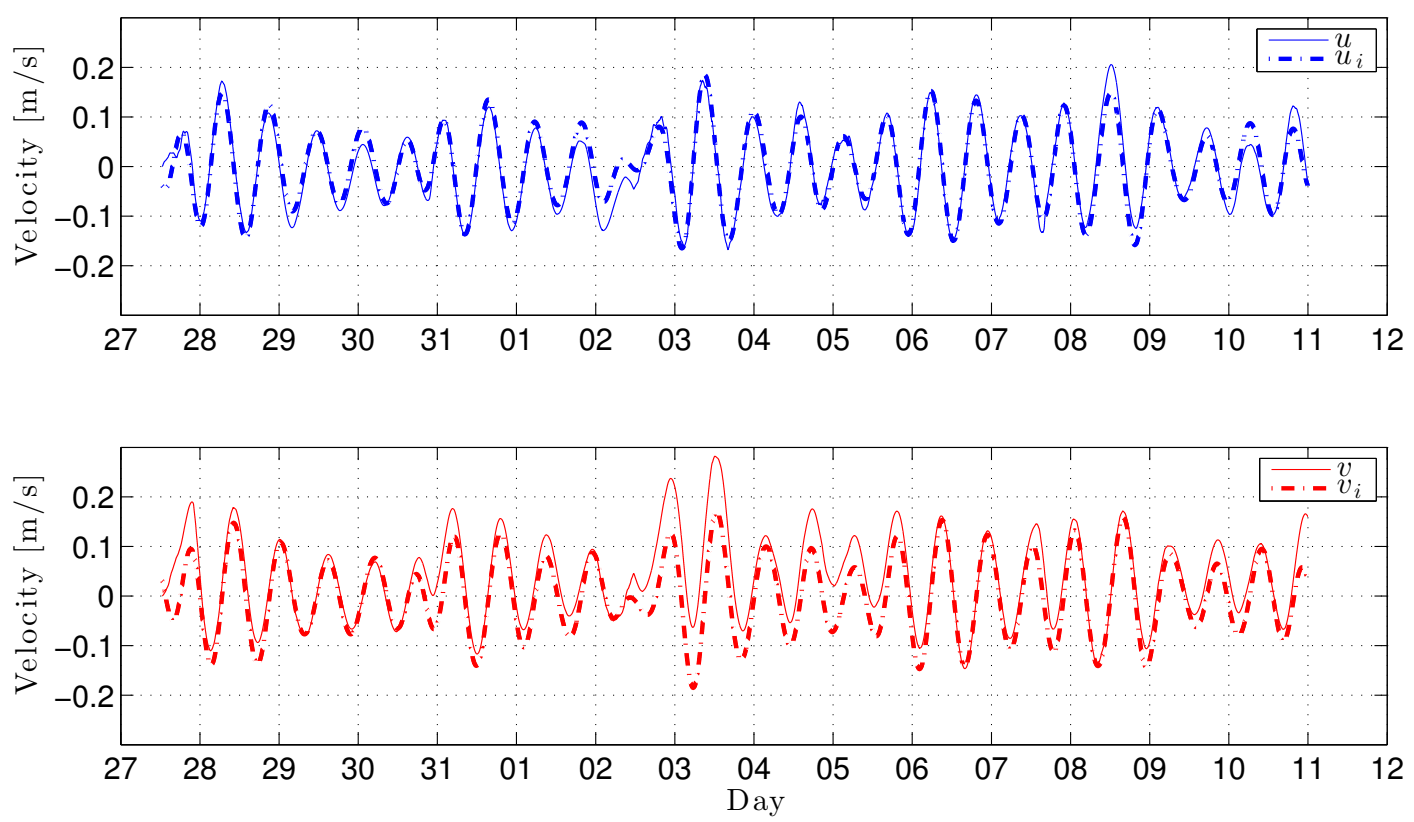

Figure 5-6: Similar to 5-5 but with $r=0.02 f$ to represent very weak damping.

\subsubsection{Reanalysis wind products}

To replicate previous studies that have estimated wind work from low-temporalresolution wind products, the European Centre for Medium-Range Weather Forecasts (ECMWF) Interim wind product (Dee et al. (2011)) is used to force the slab model. The reanalysis wind field is subsampled in space and time to match the US5 cruise track. The ECMWF product has $6 \mathrm{hr}$ temporal resolution and compares well (correlation coefficient $=0.82$ ) with the $6 \mathrm{hr}$ averaged shipboard wind time series (Fig. 5-7). The subsampled reanalysis wind is used to solve equation 5.4, using the same values of $H$ and $r$ as in the previous section.

A comparison of the near-inertial energy flux from the three methods: 1) shipboard winds and velocity from surface drifters, 2) slab model forced with shipboard winds (5min), 3) slab model forced with reanalysis winds (6hr resolution) is shown during the US5 cruise (Fig. 5-8). Of the three methods, the slab model forced with reanalysis winds gives the weakest $E_{i}$ flux. The inertial band kinetic energy $K E_{i}=\frac{1}{2}\left(u_{i}^{2}+v_{i}^{2}\right)$ is 5 times larger when forced by the high resolution shipboard wind record than the reanalysis wind product. Estimates of $E_{i}$ flux from the slab model and with drifter velocities share the shipboard wind forcing and notably the drifter velocity 

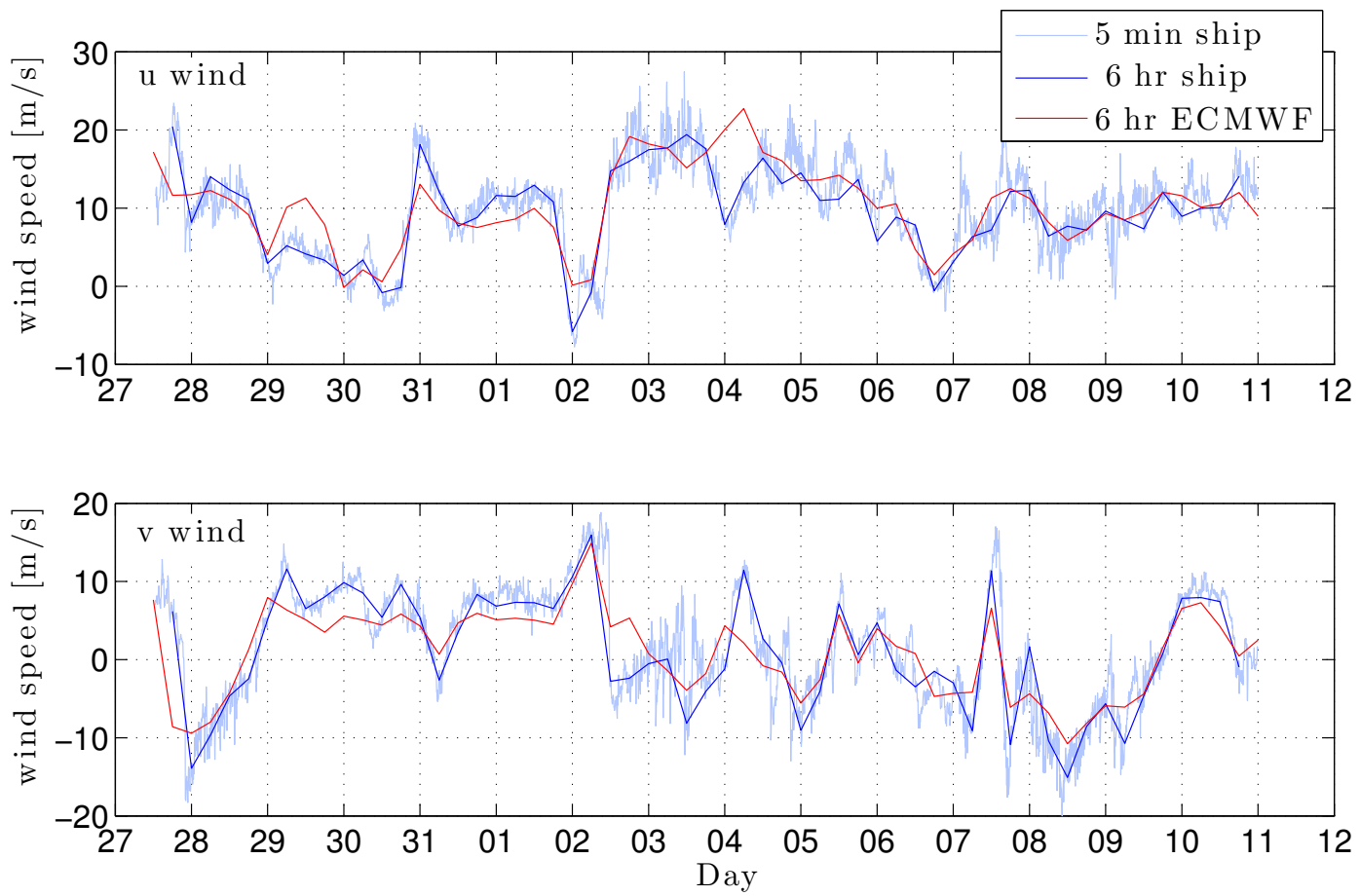

Figure 5-7: a) Zonal and b) meridional components of the wind vector measured from the ship (blue) and from the ECMWF reanalysis product (red). The wind product is well correlated to the record from the ship's mast.

was not collocated to the ship during the experiment. Nevertheless, the two methods provide insight into the velocity fields on scales smaller than a synoptic deformation radius. The two records of $E_{i}$ have similar magnitudes during strong forcing events, far exceeding the values obtained using the reanalysis product (Fig. 5-8). A summary of the RMS inertial velocities, mean inertial band kinetic energy $\left(K E_{i}\right)$, and RMS inertial energy flux $E_{i}$ is given in Table 5.1.

Table 5.1: Summary of methods used to estimate the inertial energy flux, $E_{i}, * 2 \mathrm{~d}$ refers to the same wind record as method 2, but decimated to 6-hour temporal resolution.

\begin{tabular}{|c|c|c|c|c|}
\hline Method & $r[\mathbf{1} / \mathbf{s}]$ & RMS $\vec{u}_{i}[\mathbf{c m} / \mathbf{s}]$ & $\left\langle K E_{i}>\left[\mathbf{m}^{2} / \mathbf{s}^{2}\right]\right.$ & RMS $E_{i}\left[\mathbf{m W} / \mathbf{m}^{2}\right]$ \\
\hline 1 & N/A & 7.5 & 0.56 & 5.4 \\
\hline 2 & $0.15 f$ & 3.6 & 0.25 & 4.3 \\
\hline $2 \mathrm{~d}^{*}$ & $0.15 f$ & 3.8 & 0.28 & 4.89 \\
\hline 3 & $0.15 f$ & 1.6 & 0.05 & 0.81 \\
\hline
\end{tabular}

The weak values of $E_{i}$ from the slab-model forced by reanalysis winds have been attributed to a lack of temporal variance relative to the local inertial period, 14 hours 


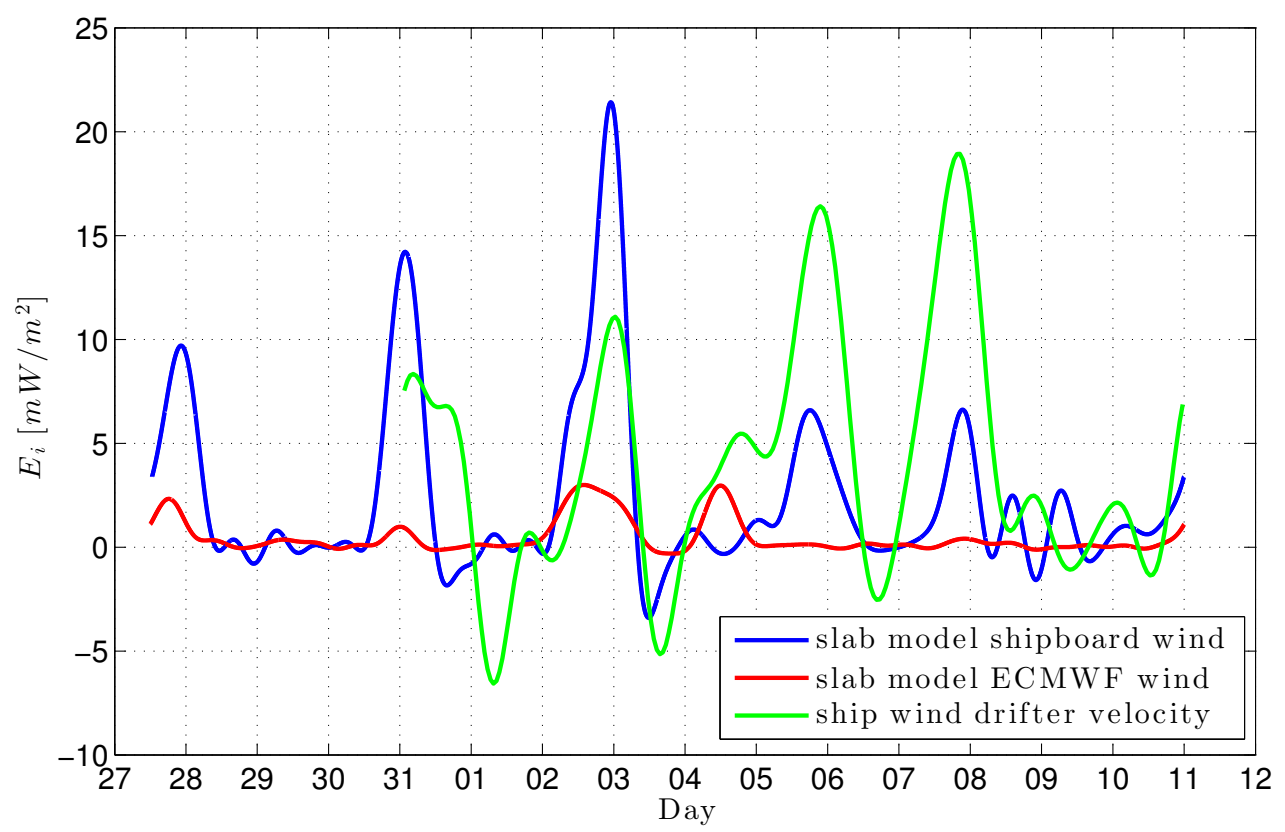

Figure 5-8: Timeseries of inertial energy flux from three methods described in the text. The slab model forced with reanalysis winds gives the weakest near-inertial flux of the methods studied.

at the study latitude (e.g. Alford (2003)). To test this, we introduce method $2 \mathrm{~d}$ (Table 5.1) which is the slab model forced by the shipboard wind record decimated to $6 \mathrm{hr}$ resolution. The largest difference between $E_{i}$ from the slab model forced by decimated US5 winds and the ECMWF winds occurs at the highest wind speeds (Fig. 5-9). This suggests that $E_{i}$ may be more sensitive to the amplitude of the wind forcing than the temporal resolution. The decimated shipboard wind record (method 2d) results in similar values of RMS $\vec{u}_{i},\left\langle K E_{i}\right\rangle$, and RMS $E_{i}$ to method 2 (Table 5.1), while method 3 yields significantly weaker values. Kilbourne and Girton (2015) posited that gridded winds are unable to accurately produce the phasing of the observed wind. Further, the mixed layer response during the passage of the storm can lead to errors in the slab model estimate of $E_{i}$. Here we conclude that the reduced amplitude of the ECMWF winds relative to the shipboard record leads to weaker values of $E_{i}$ during strong forcing events.

This section has shown that the slab model forced by shipboard winds gives weaker $E_{i}$ than those estimated from two methods using high-resolution in-situ data collected in the Southern Ocean. This result may have important implications for global maps of $E_{i}$ that have weak values at high-latitudes (e.g. Alford (2003)). 


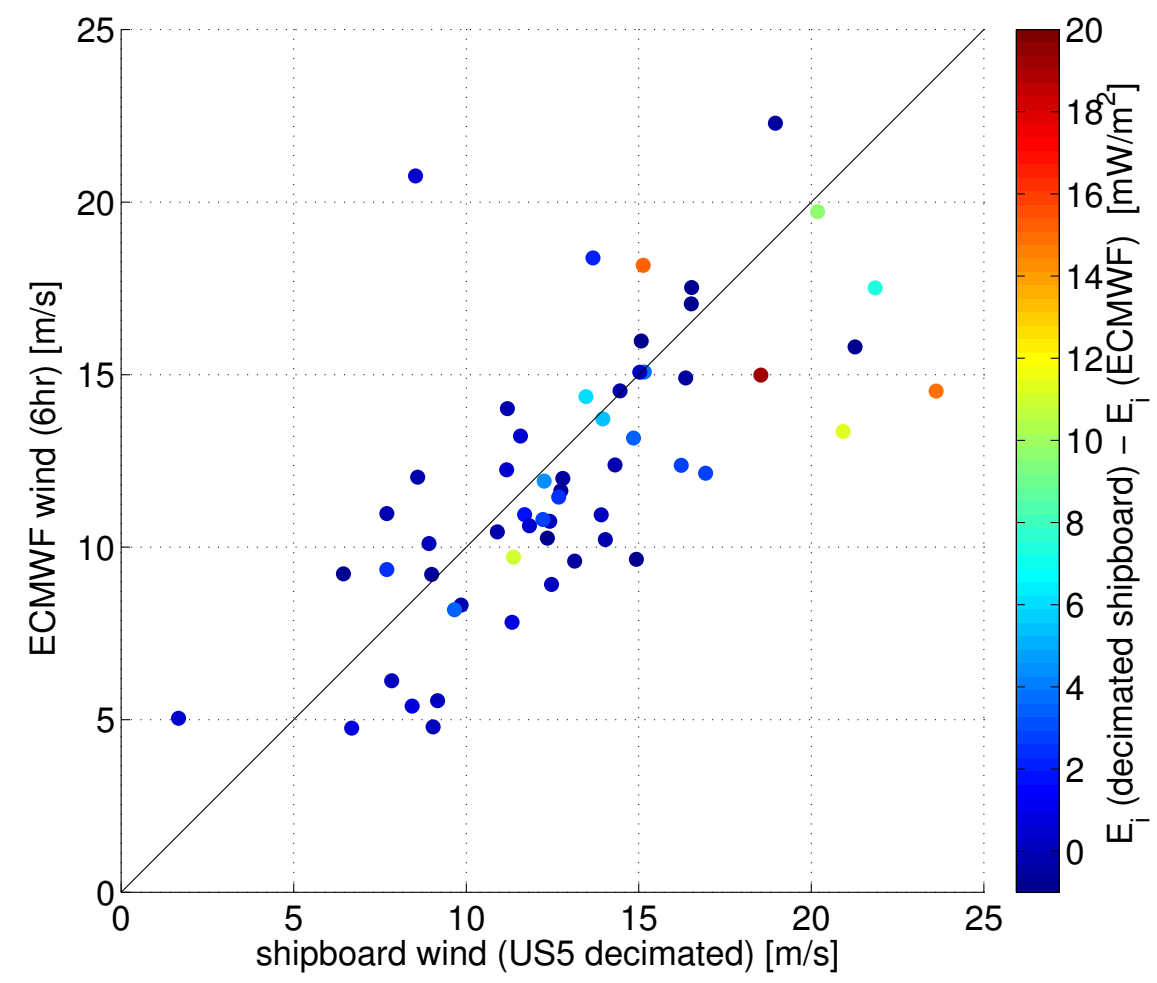

Figure 5-9: Comparison of the decimated shipboard wind record to the ECMWF wind record where colors represent the difference in the inertial energy flux from the slab model between the two methods. The laregest discrepancies occur at the highest wind speeds.

\subsection{Kinetic energy and dissipation}

The fate of the near-inertial energy that enters the mixed layer from the winds is considered by studying the observed velocity and dissipation rates in the upper $2 \mathrm{~km}$ from the US2 and US5 cruises (see section 1.5.1 and 1.5.2 for data description). To isolate ageostrophic motions, an equivalent barotropic mode is removed from the LADCP velocity component that is in the direction of the depth-averaged current, which is assumed to be the direction of the mean geostrophic flow as the ACC passes through Drake Passage. The equivalent barotropic structure function (Fig. 5-10b) is estimated as $\mu(z)=\frac{<\|\mathbf{u}(x, y, z, t)\|>}{<\|\mathbf{u}(x, y, 200, t)\|>}$. The geostrophic current vector is compared to collocated Aviso geostrophic current vectors. The agreement (Fig. 5-10a) is statistically significant with a correlation coefficient of 0.83 . 

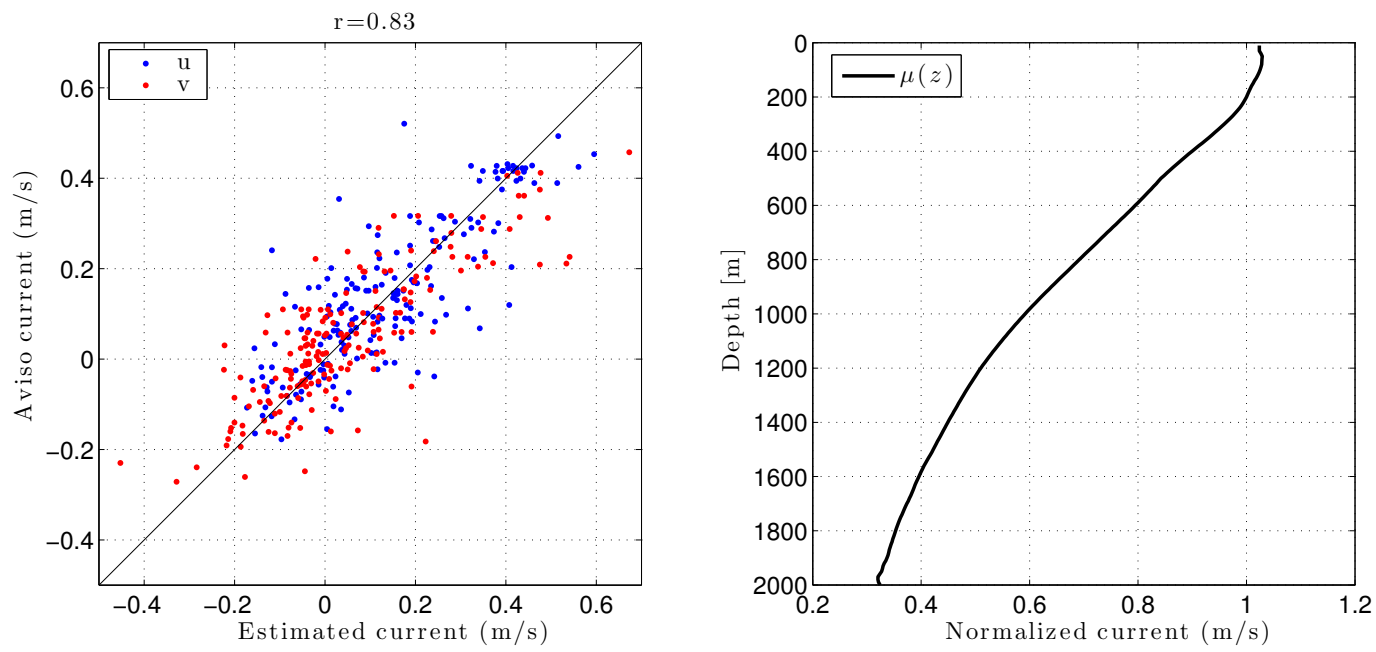

Figure 5-10: a) AVISO geostrophic velocities vs. estimated geostrophic velocities computed by removing b) the equivalent barotropic vertical structure function, $\mu(z)$ from each profile.

To estimate the inertial component of the ageostrophic currents, we consider two short time series of data when the ship was held on station for approximately 18 hours on Nov. 9 (6 LADCP stations), and 21 hours on Nov. 10 (7 stations). A least squares fit of an inertial period sinusoid is applied to the time series at each depth. The total zonal and meridional current, residual (ageostrophic) current, and inertial fit are shown for an example station in the time series (Fig. 5-11). $80 \%$ of the ageostrophic current variance is resolved by the inertial fit during these measurements, suggesting that the ageostrophic energy is dominated by inertial motions following energetic wind events.

Internal lee-waves generated at bottom topography may also be radiating into middepths. The internal lee-wave band is characterized by waves with intrinsic frequencies between 1.17f-7f. Additionally, Nikurashin and Ferrari (2010) showed that leewave generation produces inertial motions that contribute additional energy in the near-inertial band. Because surface generated NIWs likely to be dominantly downward propagating, and bottom generated lee waves upward propoagating, we consider a rotary spectral analyses are performed on the vertical shear computed from the ageostrophic current profiles in $750 \mathrm{~m}$ depth bins centered at $625 \mathrm{~m}, 1125 \mathrm{~m}$, and $1625 \mathrm{~m}$. The profiles are separated to study spatial and temporal patterns as a function of depth. Clockwise-with-depth variance corresponds to upward-propagating internal wave energy. In the SE Pacific where water depth exceeds $5000 \mathrm{~m}$ and the 

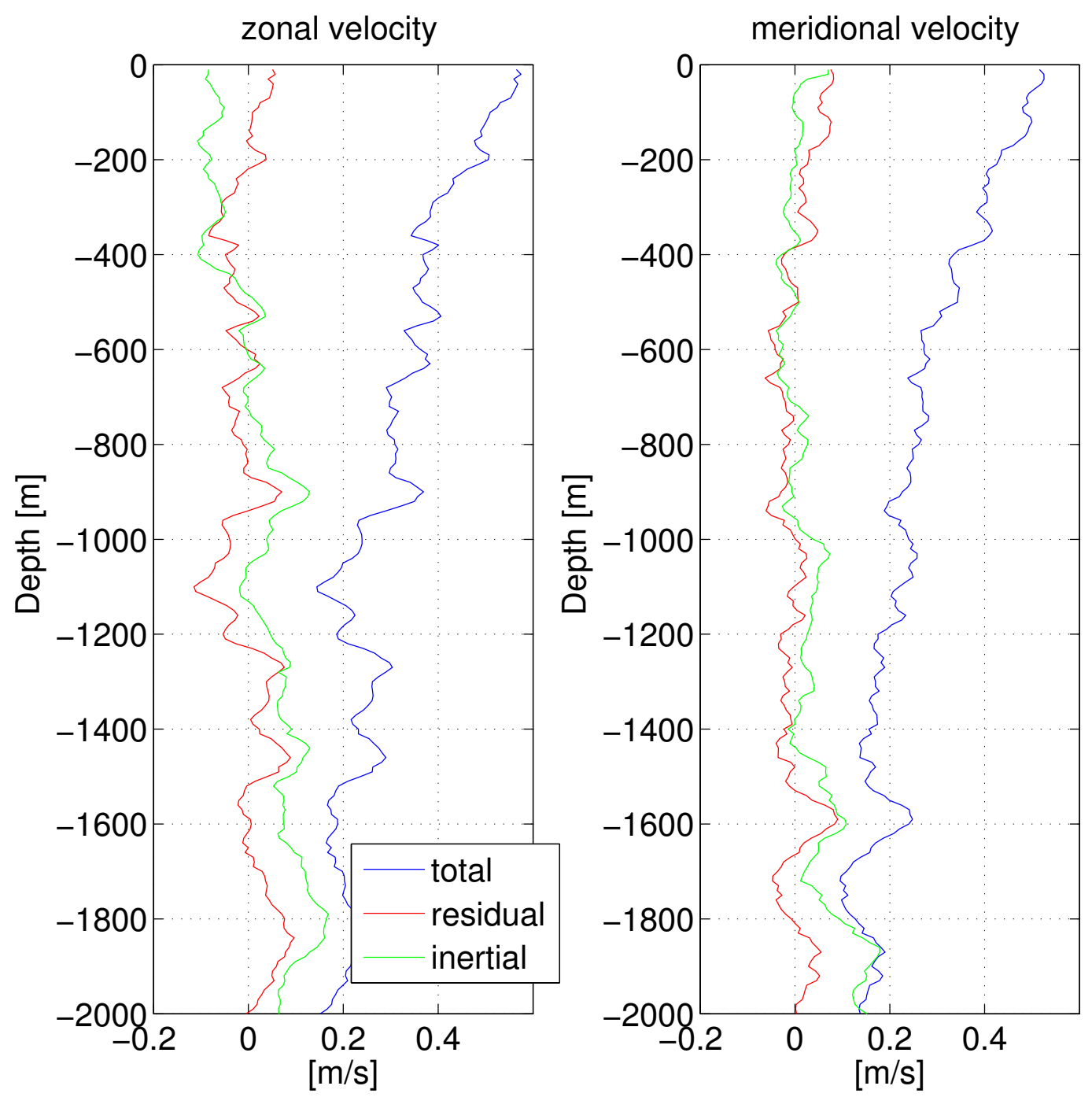

Figure 5-11: The total (blue), residual (red) and inertial (green) velocities from a representative profile in the time series. The left panel shows the zonal component of the velocity and the right panel the meridional component. The inertial fit is estimated for two sets of timeseries stations during US5. The velocity components are fit to cosine and sine waves with inertial periods as a function of depth. 
topography is smooth, the velocity measurements are expected to be free of lee-wave generation. In regions of smooth, deep topography over the cruise track, the observed vertical shear, normalized by the average buoyancy frequency, is indeed weak (Fig. $5-12 \mathrm{a}, \mathrm{b}, \mathrm{c})$. Shear that corresponds to upward propagation (CW) with depth is weak at all depths, whereas enhanced CCW energy occurs at 100-200m wavelengths at all depths. These patterns suggest a surface energy source provides the energy available for mixing in this region.

In Drake Passage, however, vertical shear with a CW polarity is enhanced in the deepest bin, consistent with upward radiating lee-wave energy (Fig. 5-12f,i). CCW energy is weak in the upper $1000 \mathrm{~m}$ during US2 (Fig. 5-12e). The US5 data in Austral spring are the most energetic, with excess CW energy in $1125 \mathrm{~m}$ and $1625 \mathrm{~m}$ bins, but with comparable values of CCW energy in the upper most bin (Fig. 5$12 \mathrm{~h}, \mathrm{i}$ ). Enhanced upward radiating energy is observed at depths as shallow as $750 \mathrm{~m}$ in Drake Passage, suggesting that lee-waves have significant non-local effects on mixing patterns in this region.

To test whether inertial band kinetic energy relates to turbulence levels, we compare the inertial energy flux to the depth-integrated dissipation in the mixed layer and in the thermocline at mid-depths. In the mixed layer, the values of $E_{i}$ estimated from the slab model forced by shipboard winds are compared to integrated dissipation rate between $10-50 \mathrm{~m}$. The majority of the values of integrated dissipation in the mixed layer are larger than $E_{i}$. This is consistent with the fact that additional processes such as wave-breaking are active in the mixed layer. In the mid-depth region, between 200-2000m, the inertial energy flux is estimated as the depth average of the ageostrophic energy density $\left(K E_{r}=\frac{1}{2} \rho\left(u^{\prime 2}+v^{\prime 2}\right)\right)$ multiplied by a vertical group velocity of $40 \mathrm{~m} /$ day taken from a study by Kilbourne and Girton (2015). Stations with depths shallower than $2500 \mathrm{~m}$ were excluded. The estimates are separated by upstream and Drake Passage and US2 and US5 cruises to show spatial and temporal variability. The estimate of $E_{i}$ is crude, but $E_{i}$ and the integrated dissipation at mid-depths have a correlation coefficient of 0.61 . The largest values of integrated dissipation and $E_{i}$ are found in Drake Passage, while the upstream values are weaker.

Probability density functions of the dissipation rate between 200-2000m show the enhancement in Drake Passage (Fig. 5-14). Upstream of Drake Passage, the measurements of dissipation taken in the SE Pacific are the weakest in the dataset below the mixed layer (Fig. 5-14a). With no bottom source of internal wave energy and a 

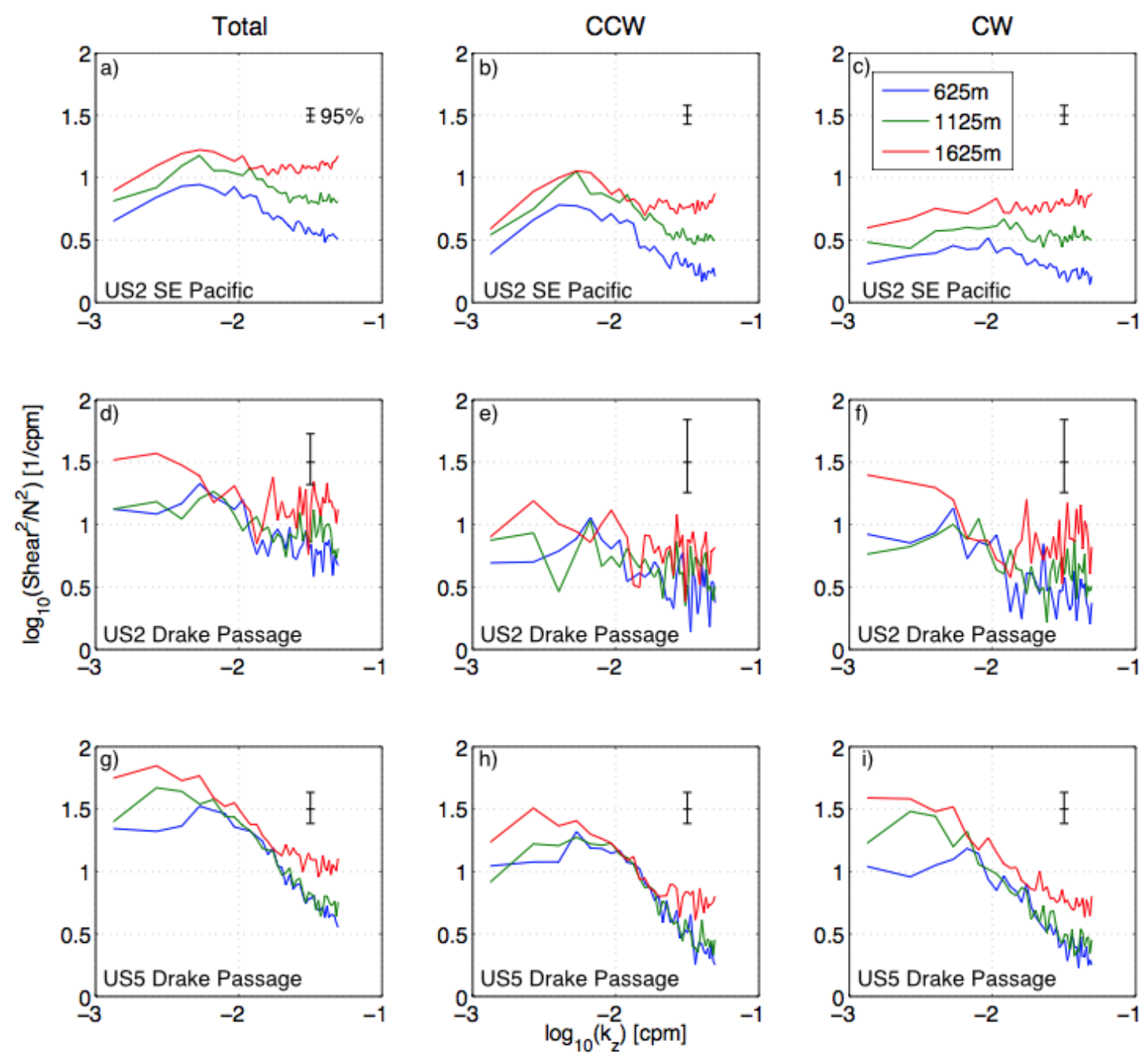

Figure 5-12: Power spectra of vertical shear normalized by mean buoyancy frequency in $750 \mathrm{~m}$ vertical bins. The vertical columns are total energy (a,d,g), counter-clockwise with depth (b,e,h), and clockwise with depth $(\mathrm{c}, \mathrm{f}, \mathrm{i})$ separated by space (upstream vs. Drake Passage) and season (Austral summer vs. Austral spring).

strongly stratified layer between 50-150m in Austral summer (Fig. 5-14d), the interior (between 200-2000m) is isolated from the atmosphere and is relatively quiet in terms of dissipation rates. In Drake Passage, the same stratified layer exists in Austral summer (Fig. 5-14e), presumably acting to inhibit downward internal wave energy and therefore turbulence levels in the interior relative to Austral spring (Fig. 5-14b,c). The stratification is estimated over a $50 \mathrm{~m}$ running interval and the enhancement at the base of the mixed layer in Austral summer is attributed to restratification due to seasonal heating. This layer erodes in the winter and into the spring, leaving the 

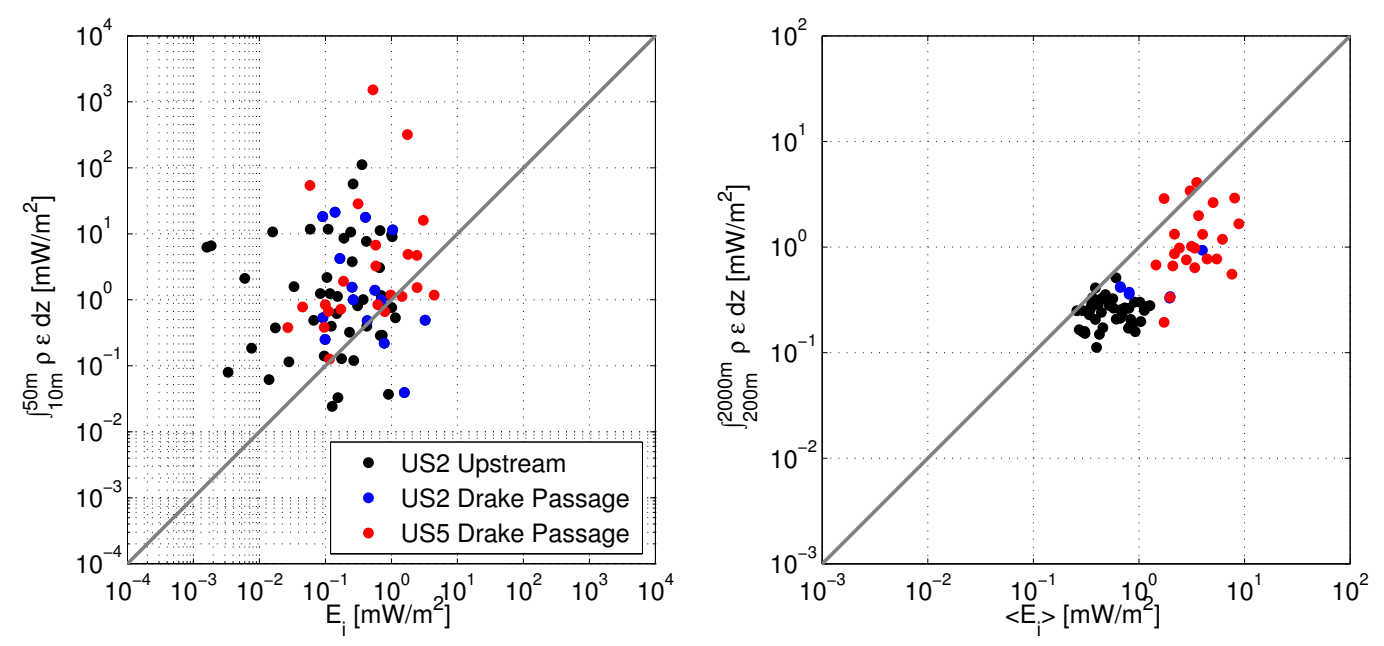

Figure 5-13: Inertial energy flux vs. integrated dissipation in the mixed layer (left panel) and at mid-depth (right panel). Integrated dissipation rates are large in the mixed layer, often exceeding values of $E_{i}$. Enhanced ageostrophic energy and dissipation are found at mid-depths during Austral spring in Drake Passage (red dots) and summer (blue dots). The weakest values are found upstream of the Passage in Austral summer (black dots).

water column weakly stratified. As a result, less wind energy is required to deepen the mixed layer in the absence of the strong near-surface stratification. 

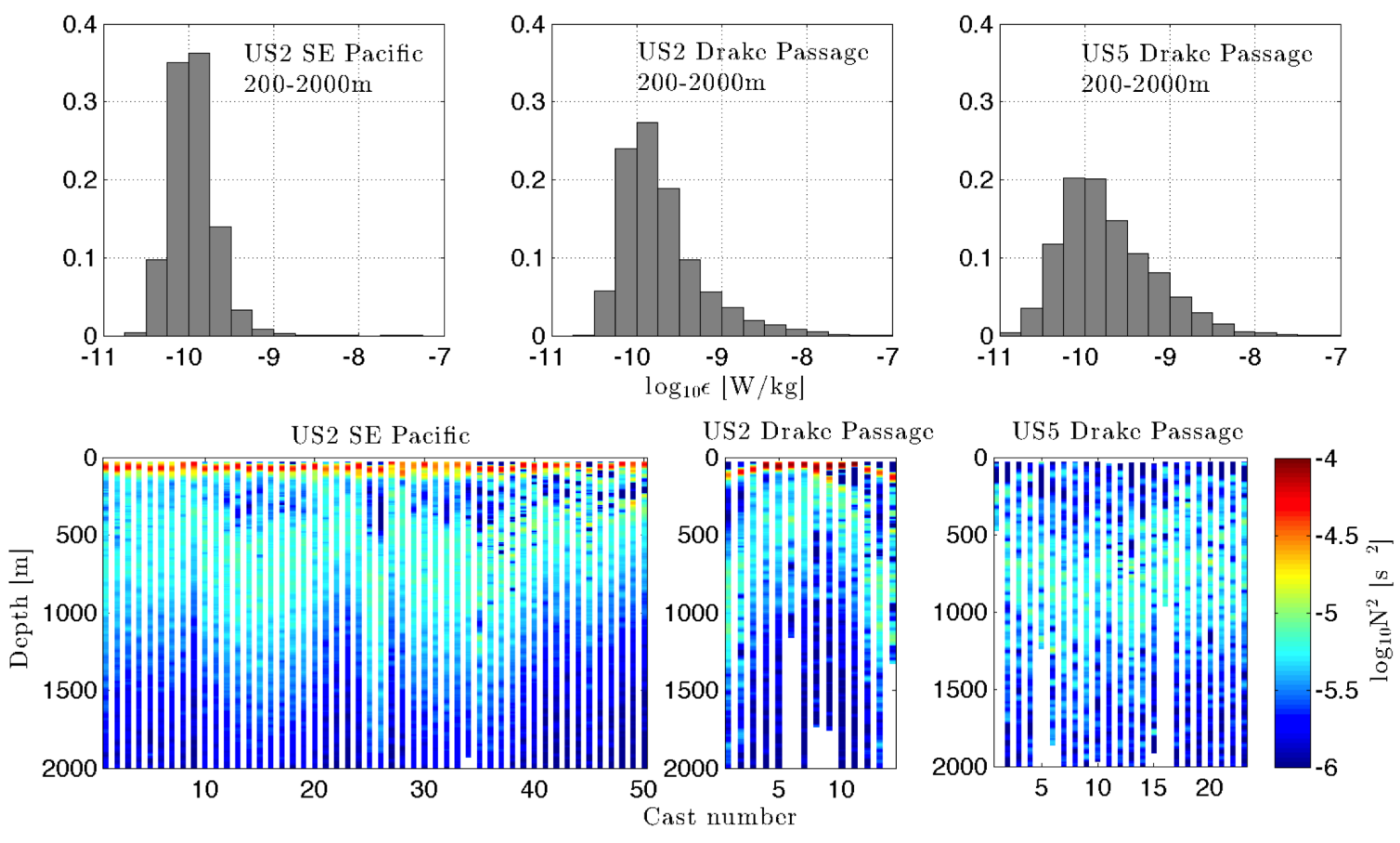

Figure 5-14: PDF's of dissipation (a,b,c) in the 200-2000m range separated spatially and seasonally. Values of dissipation in the SE Pacific are near-background throughout the region below the mixed layer. The stratification profiles $(\mathrm{d}, \mathrm{e}, \mathrm{f})$ show a strongly stratified layer in Austral summer which had eroded during the Austral Spring field experiment.

\subsection{Discussion}

The lack of air-sea flux and turbulence measurements in the Southern Ocean has made it difficult to study the oceanic response to extreme wind forcing. The region is dominated by strong zonal winds superposed with low-pressure systems that bring polar air equatorward driving turbulent heat loss. To quantify the near-inertial energy flux to mixed layer motions, three methods are used. Two of the methods using high resolution (5min) wind data from a shipboard anemometer give a five times larger inertial band kinetic energy response when compared to a similar estimate using a 6hour reanalysis product. These results suggest that estimates of wind work on inertial motions in the region based on reanalysis products may be biased low, especially during strong storm forcing. While direct measurements of winds are rare, they are necessary to update the estimates of energy flux and to better understand the energy budget in this region.

Based on the US5 shipboard dataset, we seek an estimate of the amount of near- 
inertial energy in the mixed layer that radiates downward as NIWs. To diagnose the inertial component of the flow below the mixed layer, an equivalent barotropic mode is removed from the LADCP velocity structure to isolate higher frequency motions. The ageostrophic flow kinetic energy at mid-depth is related to the integrated dissipation, with larger values of both found in Drake Passage compared to upstream. To contexualize the dissipation rates, consider a profile of $\epsilon=10^{-10} \mathrm{~W} / \mathrm{kg}$, a background value. The integrated dissipation rate, $D=\int_{2000 \mathrm{~m}}^{200 \mathrm{~m}} \rho \epsilon d z=0.18 \mathrm{~mW} / \mathrm{m}^{2}$. This value is consistent with the mid-depth rates observed in the SE sector, upstream of Drake Passage during US2. Assuming a profile of $\epsilon=10^{-9} \mathrm{~W} / \mathrm{kg}$ would give $\mathrm{D}=1.8 \mathrm{~mW} / \mathrm{m}^{2}$, a value that exceeds almost all observed values of $\mathrm{D}$ in this dataset. This result shows that values of diapycnal mixing at mid-depth in Drake Passage are weak relative to rates in the mixed layer and in the deep ocean close to rough topography. This supports the large-scale picture of the Southern Ocean overturning where the shallower cell is largely adiabatic.

The role of near-inertial waves radiating from the surface to mid-depth appears to be a weak source of mixing based on the US2 and US5 datasets. Additional data are needed to assess the seasonality of mid-depth mixing in the SW Pacific. Of the 7 DIMES cruises in the region, 6 were in Austral spring. In this season, a strong layer of stratification may influence the downward-penetration of near-inertial energy, consistent with recent modeling results (Rath et al. (2014)). We attribute enhanced values of mix-depth mixing in Drake Passage relative to upstream in part to nonlocal (vertical) effects of lee-waves generated at rough topography. 


\section{Chapter 6}

\section{Discussion and Conclusions}

\subsection{Dynamical regimes in the Southern Ocean}

This thesis analyzes in-situ data collected in Austral Spring and Summer in the Drake Passage region of the Southern Ocean to study processes that support turbulence and mixing. In the surface boundary layer, winds, buoyancy fluxes, and wave processes contribute to strong mixing rates which may vary by season. Below the seasonal thermocline and away from rough topography, shear-driven mixing is weak. Between 200-1000m, strong interleaving of temperature and salinity supports double-diffusive instability and enhanced mixing rates relative to estimates based on internal wave shear. In the deep ocean, turbulent mixing is enhanced locally where frontal flows interact with rough topography, consistent with studies such as St. Laurent et al. (2012). The nonlocal effects of lee-waves are still an active topic of research. There is evidence for upward radiating energy in the upper $1 \mathrm{~km}$, however, refraction at critical layers, for example, could be responsible for this signal.

\subsection{Mixing efficiency}

The buoyancy flux in the global ocean is dependent on the efficiency of mixing, $R_{f}$, which describes the amount of turbulent kinetic energy converted to potential energy

in stratified flows. For shear instability, the parameter $\Gamma^{(t)}=\frac{R_{f}}{1-R_{f}}$ is commonly taken to be a constant $\Gamma^{(t)}=0.2$. This value is ubiquitously used in Osborn (1980)'s model 
for turbulent mixing rates

$$
\kappa_{\rho}=\frac{\Gamma^{(t)}<\epsilon>}{<N^{2}>}
$$

However, recent studies have shown that $\Gamma^{(t)}$ may vary from the canonical 0.2 in the global ocean. In particular, there has been an effort to parameterize $\Gamma^{(t)}$ in terms of the buoyancy Reynolds number, $R e_{b}=\frac{\epsilon}{\nu N^{2}}$, and the gradient Richardson number, $R i_{g}=\frac{N^{2}}{(\partial u / \partial z)^{2}}$ (e.g. Shih et al. (2005), Ivey et al. (2008), Mater and Venayagamoorthy (2014)). We estimate $R i_{g}$ and $R e_{b}$ using data from the US5 expedition and separate the depth regime associated with interleaving (Fig. 6-1a, 6-2a) from the deep ocean (Fig. 6-1b, 6-2b), where shear instabilities associated with internal wave breaking are dominant. The largest values of $\epsilon$ and $\chi$ are associated with large buoyancy Reynolds numbers and small Richardson numbers in the deep ocean. In the interleaving region, large values of $\chi$ show no dependence on $R i_{g}$ or $R e_{b}$. In particular, large values of $\chi$ are seen at all values of Richardson and the buoyancy Reynolds number, consistent with the hypothesis that thermal dissipation rates are driven by double-diffusive processes governed by the density ratio $R_{\rho}$.
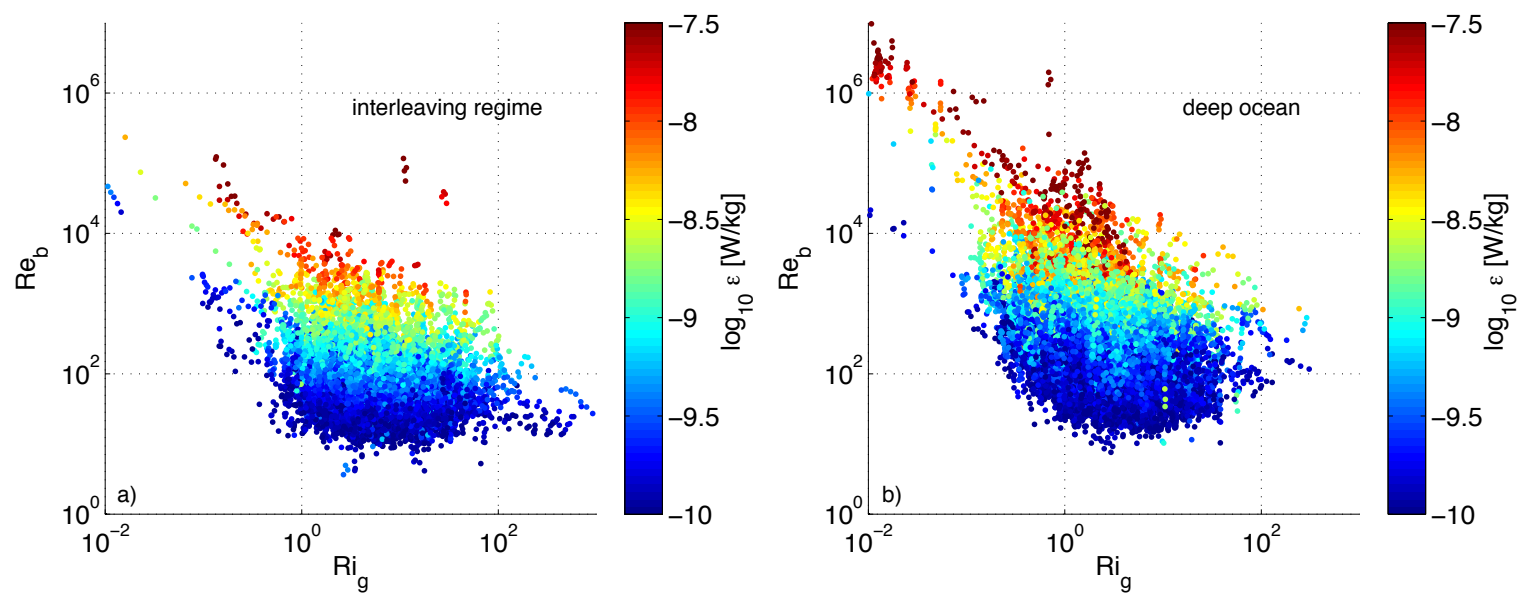

Figure 6-1: Scatter plots of $R i_{g}$ vs. $R e_{b}$ in the a) interleaving regime (200-1000m) and b) deep ocean $(\mathrm{z}>1000 \mathrm{~m})$. The data points are colored by the value of $\epsilon$ with values of $\epsilon<10^{-10}$ removed from the analysis. 


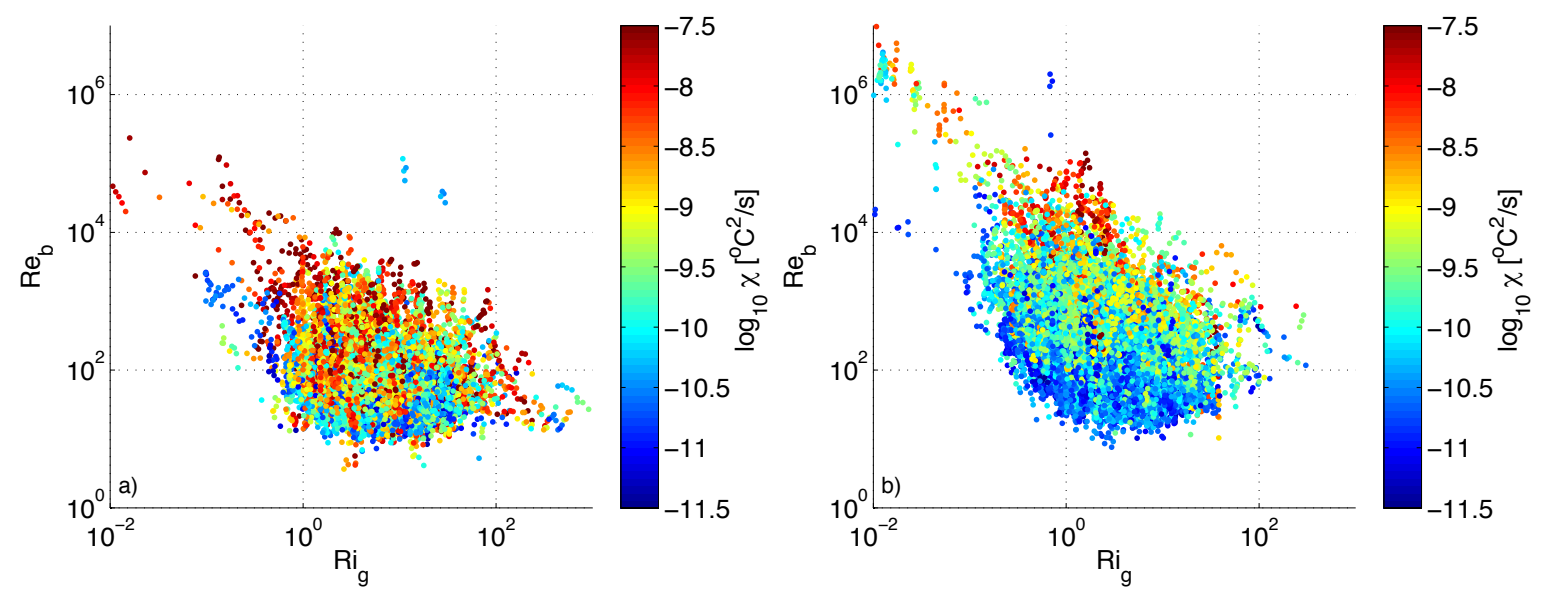

Figure 6-2: The same as Fig. 6-1 except colors show the value of $\chi$.

The $(t)$ superscript refers to the fact that turbulence is the dissipative mechanism. When this assumption is invalid, the dissipation ratio, $\Gamma=\frac{\langle\chi\rangle\left\langle N^{2}\right\rangle}{2\langle\epsilon\rangle\left\langle T_{z}\right\rangle^{2}}$ described in Chapter 3, has been used to study the efficiency of mixing. This parameter can be written in terms of the diffusivities of temperature and density as

$$
\Gamma=\frac{R_{f}}{1-R_{f}} \frac{\kappa_{T}}{\kappa_{\rho}}
$$

In high Reynolds number turbulence, $\kappa_{T}=\kappa_{\rho}$ and $\Gamma^{(t)}=\Gamma$. In the deep ocean below the interleaving regime, we estimate $\Gamma$ where turbulent mixing driven by internal wave breaking is thought to be the dominant turbulent mechanism. The values of $\Gamma$ are estimated for the highest quartile of values of $\epsilon$, where the errorbars are $95 \%$ confidence intervales using a bootstrap method (Fig. 6-3). The values of $\Gamma$ are generally close to 0.2 , consistent with previous studies based on internal wave driven mixing (Moum (1996)). 


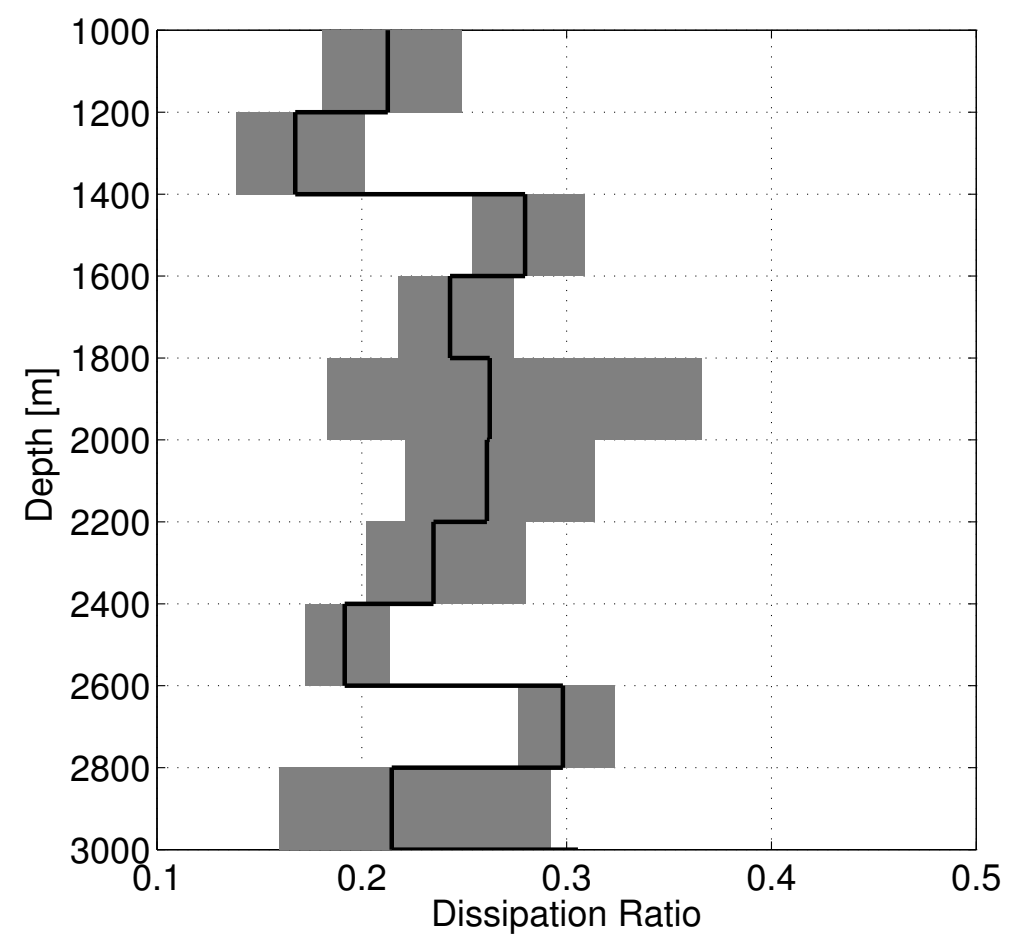

Figure 6-3: Estimates of the dissipation ratio, $\Gamma$, from statistics of US5 below the interleaving features. The values are estimated for the upper quartile of the dataset based on values of $\epsilon$.

\subsection{Autonomous sampling strategies}

While DIMES provided the first direct measurements of Southern Ocean mixing rates, the study was focused on deep and abyssal waters below 1000-m depth. The nature of the DIMES surveys was not ideal for sampling the spatial and temporal scales of processes that directly respond to forcing from the atmosphere. Critically, microstructure measurements of turbulence from ships can only be made in relatively calm conditions. Consequently, there is a significant fair-weather bias in the DIMES dataset of upper ocean turbulence. To date, there has not been an effort to collect upper ocean turbulence data in coincident with air-sea fluxes in the Southern Ocean. There are very few in-situ air-sea flux measurements in this region and shipboard measurements are subject to the aforementioned fair-weather bias. Float data, such as those collected as part of the DIMES study (Kilbourne and Girton (2015)), lack coincident air-sea meaurements of any kind, and rely entirely on climatological products for estimates of forcing. Model-data comparisons of the thickness of the boundary layer 
in the Southern Ocean have been shown to have errors often exceeding 90\% (Belcher et al. (2012)). Model validation in the high-wind regime has been compromised by a lack of direct measurements of both ocean turbulence and air-sea fluxes.

Our understanding of the processes driving enhanced mixing in the upper $1000 \mathrm{~m}$ has been limited by the nature of existing data. To fill these gaps, an experiment is proposed to collect a dataset that will inform parameterizations of mixing processes and serve to validate large-scale models in a region that is severely under-sampled.

A proposed experimental study would take advantage of autonomous assets such as surface drifters and ocean gliders. In particular, a constellation of these assets engineered to include meteorological, wave and turbulence sensors would provide an unprecedented view into the relationship between atmospheric forcing and oceanic mixing. This type of sampling pattern spawns from the gaps in this thesis and the lack of data in this region. In particular, the hypotheses listed below motivate the use of autonomous assets in this region:

\section{Double-diffusion is active in this region when shear-processes are weak.}

The rates of vertical diffusivity are underestimated by traditional implementations of the Osborn method using a mixing efficiency of 0.2. The temperature, salinity, and turbulence data from the Slocum diving to $1000 \mathrm{~m}$ will give a high resolution dataset for studying the impact of double-diffusion in the water column. We will be able to assess the relative importance of shear-driven and double-diffusive mixing motivating an assessment of current mixing parameterizations in models.

\section{Langmuir physics contribute significantly to turbulence in the near-} surface boundary layer in this region, due to the strong wind forcing and large surface waves.

Using wind and wave data from the Wave Glider coincident with microstructure measurements from a shallow-diving Slocum, we will be able to assess the impacts of swell and Langmuir processes supporting turbulence in the surface boundary layer. Notably, we will be able to estimate the Langmuir number: $L a=\left(u^{*} / u_{S}\right)^{1 / 2}$, where $u_{S}$ is the Stokes drift vector associated with the surface waves and $u *$ is the surface friction velocity. $L a$ distinguishes the relative importance of the two production terms and modeling studies (ie. Belcher et al. (2012)) have shown that Langmuir turbulence dominates when $L a<0.3$. Wave 
spectral data will be analyzed following studies such as D'Asaro (2014)). Additionally, using radiation data from the drifters we will study the impact of diurnal forcing relative to strong mechanical forcing by the winds. This will aid boundary layer mixing schemes in global models as well as process modeling efforts at the submesoscale.

\section{The inertial energy input into the mixed layer has been underesti- mated by studies that use reanalysis wind products.}

Most studies of inertial energy input (Alford (2003),Kilbourne and Girton (2015)) use a reanalysis wind product with a 6-12 hour resolution. The inertial period in this region is $\sim 14$ hours such that the reanalysis products are unable to resolve the full amplitude and frequency of strong storms. A comparison of all of the shipboard wind data from the DIMES project to the 6 hourly ERA-Interim reanalysis wind illustrates the systematic underestimation by the product.

Questions and hypotheses that have been elucidated by this thesis are the focus of future work in this region. Notably, the advent of autonomous platforms will provide a dataset that gives new scientific perspective to this remote and energetic region of the global ocean.

\subsection{Summary}

The findings from this thesis are summarized as follows. Direct measurements of turbulent dissipation in the Drake Passage region of the Southern Ocean are enhanced in regions where frontal flows interact with rough topography. Evidence of upward radiating energy consistent with lee-wave generation is evident at depths as shallow as $1 \mathrm{~km}$. The degree to which lee-waves contribute to non-local mixing rates is still a topic for further research as observational platforms become better equipped to handle the conditions in the Southern Ocean.

Previous estimates of mixing rates before the DIMES experiment were predominately based on finestructure parameterizations. These methods rely on the assumption that mixing rates away from boundaries are driven by internal wave breaking. While previous studies have noted that thermohaline variance can bias finestructure parameterizations (e.g Kunze et al. (2006)), this work presents the first effort to identify the bias in the interleaving region of the Southern Ocean. Distinguishing internal 
wave strain from watermass interleaving is an important topic for for the future of finestructure parameterizations. A comparison of microstructure based mixing rates and finestructure parameterizations shows a large discrepancy in the intrusive region of the water column. In northern Drake Passage this depth range is between 200-1000m.

Intrusions in temperature and salinity are supported by water mass variability and mesoscale activity in this region. Double-diffusion was first proposed to be an active process in this region in the 1970's, however, this thesis presents the first comprehensive effort to show the implications for mixing rates. Mixing rates are found to be up to three times larger than those estimated from the conventional Osborn method. The Osborn method relies on a mixing efficiency parameter which is ill-defined in the presence of double-diffusion. Hydrographic data give evidence that this form of instability is likely prevalent throughout the Southern Ocean.

The surface boundary layer in this region is characterized by a layer of enhanced turbulent dissipation, likely consistent with breaking surface waves. This layer of active mixing is sensitive to local wind forcing and the seasonal stratification, which inhibits mixed layer deepening in the summer months. Conventional boundary layer scalings fail to represent the structure of observed turbulent dissipation, consistently underestimating the upper $15 \mathrm{~m}$ and overestimating rates below this layer. This has significant implications for climate models which must use parameterizations to represent the interactions between the atmosphere and the ocean, and the mixed layer and the interior ocean.

Finally, this work studies the energetic pathway from wind forcing to turbulent dissipation in the form of near-inertial waves radiating from the mixed layer. While there are limitations to estimating the near-inertial energy flux from observations, the use of high-resolution ship winds yield an inertial kinetic energy response five times larger than when using coarse temporal reanalysis wind products. Because the inertial period is 14 hours at the study latitude, limited variance in the near-inertial band leads to a weaker mixed layer response and consequently lower values of inertial energy flux. It is posited that most of the near-inertial energy that enters the ocean dissipates in the upper hundred meters, above the seasonal thermocline. There is very little evidence for downward radiating energy during the study period, as mixing rates are near-background and high wavenumber shear is weak.

The results of this thesis suggest that many of the mixing parameterizations are not 
appropriate for the Southern Ocean. In addition, this thesis highlights the pressing need for more observations in this region of the global ocean. Air-sea fluxes, wind and wave information, and measurements of turbulent parameters in this region will provide insight into the oceanic response to extreme forcing. Whether these episodic storm events significantly influence the interior ocean and consequently, the large scale circulation remains an open question. 


\section{Appendix A}

\section{Appendix ${ }^{1}$}

\section{A.0.1 Estimates and uncertainty associated with $\epsilon$}

Microstructure variance is estimated using spectral analysis over 1-m depth intervals of each profile record using methods well described in the literature (eg, Schmitt et al. (1988), Gregg (1999), Lueck (2002)). No spectral corrections are done to the shear spectra, as the inertial sub-range and dissipative roll-off were always well resolved (Fig. A-1). The thermal gradient spectra are well resolved out to about $20 \mathrm{~Hz}$, and issues with estimating $\chi$ are described by Gregg (1999) and Nash and Moum (1999). To account for unresolved thermal variance in the diffusive subrange, we match the variance of the resolved portion of the measured temperature spectrum $(\omega<20 \mathrm{~Hz})$ to that of the Batchelor model (Batchelor (1959), Oakey (1982)) corresponding to our measured value of $\epsilon$.

\section{A.0.2 Estimates and uncertainty associated with $\chi$}

The microstructure temperature signals are measured using FP07 probes. As described by Gregg (1999), Nash and Moum (1999), and others, these probes alias the measured signal at frequencies greater than $20 \mathrm{~Hz}$. As such, a corrective method

\footnotetext{
${ }^{1}$ This section includes text and the appendix of Merrifield, S. T., Laurent, L. S., Owens, B., Thurnherr, A. M., \& Toole, J. M. (2016). Enhanced Diapycnal Diffusivity in Intrusive Regions of the Drake Passage. Journal of Physical Oceanography, 46(4), 1309-1321. American Meteorological Society. Reproduced with permission.
} 

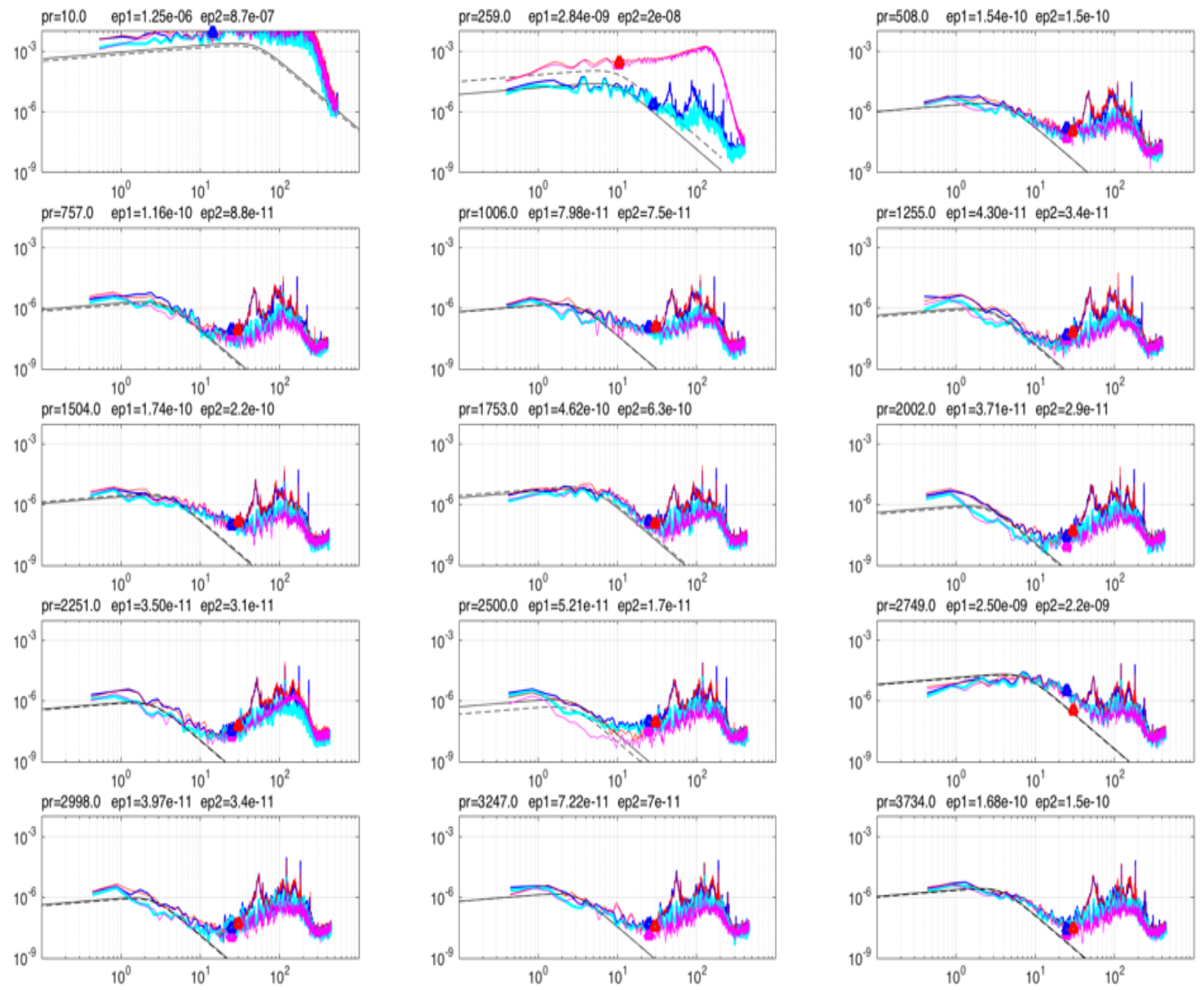

Figure A-1: Shear spectra as a function of pressure, black line represents the Nasmyth spectrum for a given value of $\epsilon$. The upper middle panel shows a case where one probe had an anomalous response, most likely due to impact with zoo plankton. In this case, only the spectral signal of the good probe is used for the estimate of $\epsilon$.

must be adopted to properly estimate temperature variance at the higher frequencies that constitute the diffusive subrange portion of the spectrum. Generally, this is done through a combination of a corrective transfer function modeled on the FP07's response, and the use of a spectral model.

For our estimates, we calculate the derivative (first difference) of the microstructure temperature signal $\frac{\partial T}{\partial t}$, and then compute its spectrum over 2 second windows in a given profile. We then 1) correct for the first difference using a transfer function, and 2) correct for probe response using the frequency response transfer function proposed by Gregg (1999) with a 5 millisecond time constant. We then integrate the variance of the spectrum out to $20 \mathrm{~Hz}$ giving us a reference variance level. Using the known value 
of $\epsilon$ for the same spectral window, we find the Batchelor spectrum that has 1) the same value of $\epsilon$, and 2) the same integrated variance level to $\omega=20 \mathrm{hz}$ as the measured reference value. We then use the value of $\chi$ that goes with that Batchelor spectrum. By using the $\omega<20 \mathrm{~Hz}$ frequency band, we are largely insensitve to the behavior of the frequency response transfer function. This, however, shifts the resolution of higher frequency variance to the spectral model of temperature fluctuations.

The assumption of a universal scalar spectrum and the estimate of the empirical scale factor, $q$, are the most significant sources of error in estimating $\chi$. We use the Batchelor (1959) model of the temperature spectrum to account for the variance of the diffusive subrange. An alternative form of the scalar variance spectrum is presented by Kraichnan (1968). The Batchelor (1959) model with a scale factor, $q=3.7$ (Oakey (1982)), is most commonly used in the literature. A discussion of the uncertainty in $q$ is presented by Nash and Moum (1999) and Nash and Moum (2002). A value of $q>3.7$ gives the Batchelor spectrum more variance for a given value of $\chi$. In the most extreme case of very high Reynolds number turbulence, Nash and Moum (2002) found half of their estimates in the range $4.2<q<7$ while Gargett (1985) argued $q$ could be as large as 12. To examine the sensitivity of our $\chi$ estimates to this uncertainty, we ran calculations for both the standard value of $q=3.7$ and the extreme value of $q=12$. We find that for values of $\chi$ that are characteristic of our data, the larger value of $q$ results in values of $\chi$ that are $20 \%$ to $30 \%$ lower than our standard estimates. We do not believe our $\chi$ estimates are biased high, as the value of $q=3.7$ is well established as the standard form of the Batchelor spectrum for typical oceanographic turbulence (Nash and Moum (1999)). However, even in the most extreme case, uncertainty in $q$ can not account for the $200 \%$ to $300 \%$ elevation of $\kappa_{T}$ relative to $\kappa_{\rho}$ in double diffusive portions of the data. 


\section{Bibliography}

Abernathey, R., Marshall, J., Mazloff, M., and Shuckburgh, E. (2010). Enhancement of mesoscale eddy stirring at steering levels in the Southern Ocean. Journal of Physical Oceanography, 40(1):170-184.

Agrawal, Y., Terray, E., Donelan, M., Hwang, P., Williams, A., Drennan, W., Kahma, K., and Krtaigorodskii, S. (1992). Enhanced dissipation of kinetic energy beneath surface waves. Nature.

Alford, M. H. (2003). Improved global maps and 54-year history of wind-work on ocean inertial motions. Geophysical Research Letters, 30(8):1424.

Alford, M. H. and Pinkel, R. (2000). Observations of overturning in the thermocline: The context of ocean mixing. Journal of Physical Oceanography, 30(5):805-832.

Alford, M. H. and Whitmont, M. (2007). Seasonal and spatial variability of nearinertial kinetic energy from historical moored velocity records. Journal of Physical Oceanography, 37(8):2022-2037.

Batchelor, G. K. (1959). Small-scale variation of convected quantities like temperature in turbulent fluid. Journal of Fluid Mechanics, 5:113-133.

Belcher, S. E., Grant, A. L., Hanley, K. E., Fox-Kemper, B., Van Roekel, L., Sullivan, P. P., Large, W. G., Brown, A., Hines, A., Calvert, D., et al. (2012). A global perspective on langmuir turbulence in the ocean surface boundary layer. Geophysical Research Letters, 39(18).

Boccaletti, G., Ferrari, R., and Fox-Kemper, B. (2007). Mixed layer instabilities and restratification. Journal of Physical Oceanography, 37(9):2228-2250.

Brainerd, K. E. and Gregg, M. C. (1995). Surface mixed and mixing layer depths. Deep Sea Research Part I: Oceanographic Research Papers, 42(9):1521-1543.

Callies, J., Ferrari, R., Klymak, J. M., and Gula, J. (2015). Seasonality in submesoscale turbulence. Nature communications, 6 .

Centurioni, L. (2011). The global drifter program. Annual Report.

Craik, A. D. and Leibovich, S. (1976). A rational model for langmuir circulations. Journal of Fluid Mechanics, 73(03):401-426. 
Cushman-Roisin, B. (1994). Introduction to geophysical fluid dynamics. Prentice Hall, Englewood Cliffs, NJ, 7632.

Cuypers, Y., Le Vaillant, X., Bouruet-Aubertot, P., Vialard, J., and Mcphaden, M. J. (2013). Tropical storm-induced near-inertial internal waves during the cirene experiment: Energy fluxes and impact on vertical mixing. Journal of Geophysical Research: Oceans, 118(1):358-380.

D’Asaro, E., Lee, C., Rainville, L., Harcourt, R., and Thomas, L. (2011). Enhanced turbulence and energy dissipation at ocean fronts. Science, 332(6027):318-322.

D'Asaro, E. A. (1985). The energy flux from the wind to near-inertial motions in the surface mixed layer. Journal of Physical Oceanography, 15(8):1043-1059.

D'Asaro, E. A. (2014). Turbulence in the upper-ocean mixed layer. Annual Review of Marine Science, 6:101-115.

D’Asaro, E. A., Eriksen, C. C., Levine, M. D., paulson, C. A., Niiler, P., and Van Meurs, P. (1995). Upper-ocean inertial currents forced by a strong storm. part i: Data and comparisons with linear theory. Journal of Physical Oceanography, 25(11):2909-2936.

D’Asaro, E. A., Thomson, J., Shcherbina, A. Y., Harcourt, R. R., Cronin, M. F., Hemer, M. A., and Fox-Kemper, B. (2014). Quantifying upper ocean turbulence driven by surface waves. Geophysical Research Letters, 41(1):102-107.

Davis, R. E. (1994). Diapycnal mixing in the ocean: equations for large-scale budgets. Journal of Physical Oceanography, 24(4):777-800.

Dee, D., Uppala, S., Simmons, A., Berrisford, P., Poli, P., Kobayashi, S., Andrae, U., Balmaseda, M., Balsamo, G., Bauer, P., et al. (2011). The era-interim reanalysis: Configuration and performance of the data assimilation system. Quarterly Journal of the Royal Meteorological Society, 137(656):553-597.

Dillon, T. (1982). Vertical overturns: A comparison of thorpe and ozmidov length scales. Journal of Geophysical Research: Oceans (1978-2012), 87(C12):9601-9613.

Dong, S., Sprintall, J., Gille, S. T., and Talley, L. (2008). Southern ocean mixedlayer depth from argo float profiles. Journal of Geophysical Research: Oceans, $113(\mathrm{C} 6): \mathrm{n} / \mathrm{a}-\mathrm{n} / \mathrm{a}$.

Drennan, W., Donelan, M., Terray, E., and Katsaros, K. (1996). Oceanic turbulence dissipation measurements in swade. Journal of Physical Oceanography, 26(5):808815.

Drennan, W. M., Kahma, K. K., and Donelan, M. A. (1999). On momentum flux and velocity spectra over waves. Boundary-Layer Meteorology, 92(3):489-515. 
Egbert, G. D., Bennett, A. F., and Foreman, M. G. (1994). Topex/poseidon tides estimated using a global inverse model.

Elipot, S. and Gille, S. T. (2009). Estimates of wind energy input to the ekman layer in the southern ocean from surface drifter data. Journal of Geophysical Research: Oceans (1978-2012), 114(C6).

Fairall, C. W., Bradley, E. F., Rogers, D. P., Edson, J. B., and Young, G. S. (1996). Bulk parameterization of air-sea fluxes in toga coare. Journal of Geophysical Research, 101(3747-3767).

Ferrari, R. and Nikurashin, M. (2010). Suppression of eddy diffusivity across jets in the Southern Ocean. Journal of Physical Oceanography, 40(7):1501-1519.

Ferrari, R. and Polzin, K. L. (2005). Finescale structure of the T-S relation in the eastern North Atlantic. Journal of Physical Oceanography, 35(8):1437-1454.

Fox-Kemper, B., Danabasoglu, G., Ferrari, R., Griffies, S., Hallberg, R., Holland, M., Maltrud, M., Peacock, S., and Samuels, B. (2011). Parameterization of mixed layer eddies. iii: Implementation and impact in global ocean climate simulations. Ocean Modelling, 39(1-2):61 - 78 .

Frants, M., Damerell, G. M., Gille, S. T., Heywood, K. J., MacKinnon, J., and Sprintall, J. (2013). An assessment of density-based finescale methods for estimating diapycnal diffusivity in the Southern Ocean. Journal of Atmospheric and Oceanic Technology, 30(11):2647-2661.

García-Nava, H., Ocampo-Torres, F. J., Hwang, P., and Osuna, P. (2012). Reduction of wind stress due to swell at high wind conditions. Journal of Geophysical Research: Oceans, 117(C11).

Gargett, A. E. (1985). Evolution of scalar spectra with the decay of turbulence in a stratified fluid. Journal of Fluid Mechanics, 159:379-407.

Garrett, C. (2001). Stirring and mixing: What are the rate controlling processes. In Proc.'Aha Huliko'a Winter Workshop, pages 1-8. DTIC Document.

Garrett, C. and Munk, W. (1972). Space-time scales of internal waves. Geophysical \&3 Astrophysical Fluid Dynamics, 3(1):225-264.

Grant, A. L. M. and Belcher, S. E. (2009). Characteristics of langmuir turbulence in the ocean mixed layer. Journal of Physical Oceanography, 39(8):1871-1887.

Gregg, M. (1999). Uncertainties and limitations in measuring $\varepsilon$ and $\chi$. Journal of Atmospheric and Oceanic Technology, 16(11):1483-1490.

Gregg, M. C. (1989). Scaling turbulent dissipation in the thermocline. Journal of Geophysical Research: Oceans, 94(C7):9686-9698. 
Gregg, M. C., Sanford, T. B., and Winkel, D. P. (2003). Reduced mixing from the breaking of internal waves in equatorial waters. Nature, 422(6931):513-515.

Holte, J., J. G. L. T. and Roemmich, D. (2010). Argo mixed layers, scripps institution of oceanography/ucsd http://mixedlayer.ucsd.edu.

Hoskins, B. (1974). The role of potential vorticity in symmetric stability and instability. Quarterly Journal of the Royal Meteorological Society, 100(425):480-482.

Ivey, G., Winters, K., and Koseff, J. (2008). Density stratification, turbulence, but how much mixing? Annual Review of Fluid Mechanics, 40(1):169.

Joyce, T. M. (1977). A note on the lateral mixing of water masses. Journal of Physical Oceanography, 7(4):626-629.

Joyce, T. M., Zenk, W., and Toole, J. M. (1978). The anatomy of the Antarctic polar front in the Drake Passage. Journal of Geophysical Research: Oceans (1978-2012), 83(C12):6093-6113.

Kilbourne, B. F. and Girton, J. B. (2015). Quantifying high-frequency wind energy flux into near-inertial motions in the southeast pacific. Journal of Physical Oceanography, 45(2):369-386.

Klocker, A. and Abernathey, R. (2014). Global patterns of mesoscale eddy properties and diffusivities. Journal of Physical Oceanography, 44(3):1030-1046.

Kraichnan, R. H. (1968). Small-scale structure of a scalar field convected by turbulence. Physics of Fluids (1958-1988), 11(5):945-953.

Kukulka, T., Plueddemann, A. J., Trowbridge, J. H., and Sullivan, P. P. (2009). Significance of langmuir circulation in upper ocean mixing: Comparison of observations and simulations. Geophysical Research Letters, 36(10).

Kunze, E., Briscoe, M. G., and Williams, A. (1990). Interpreting shear and strain fine structure from a neutrally buoyant float. Journal of Geophysical Research: Oceans (1978-2012), 95(C10):18111-18125.

Kunze, E., Firing, E., Hummon, J. M., Chereskin, T. K., and Thurnherr, A. M. (2006). Global abyssal mixing inferred from lowered adcp shear and ctd strain profiles. Journal of Physical Oceanography, 36(8):1553-1576.

Langmuir, I. et al. (1938). Surface motion of water induced by wind. Science, 87(2250):119-123.

Large, W. and Pond, S. (1981). Open ocean momentum flux measurements in moderate to strong winds. Journal of Physical Oceanography, 11(3):324-336.

Ledwell, J. R., St. Laurent, L. C., Girton, J. B., and Toole, J. M. (2010). Diapycnal Mixing in the Antarctic Circumpolar Current. Journal of Physical Oceanography, $41(1): 241-246$. 
Leibovich, S. (1983). The form and dynamics of langmuir circulations. Annual Review of Fluid Mechanics, 15(1):391-427.

Li, Q., Webb, A., Fox-Kemper, B., Craig, A., Danabasoglu, G., Large, W. G., and Vertenstein, M. (2015). Langmuir mixing effects on global climate: Wavewatch iii in cesm. Ocean Modelling, pages -.

Liu, G., Perrie, W. A., and He, Y. (2014). Ocean surface stokes drift from scatterometer observations. International Journal of Remote Sensing, 35(5):1966-1978.

Lombardo, C. and Gregg, M. (1989). Similarity scaling of viscous and thermal dissipation in a convecting surface boundary layer. Journal of Geophysical Research: Oceans (1978-2012), 94(C5):6273-6284.

Lueck, R.G., W. F. Y. H. (2002). Oceanic velocity microstructure measurements in the 20th century. Journal of Oceanography, 58(153-174).

Lumpkin, R. and Speer, K. (2007). Global ocean meridional overturning. Journal of Physical Oceanography, 37(10):2550-2562.

Marshall, J. and Speer, K. (2012). Closure of the meridional overturning circulation through southern ocean upwelling. Nature Geoscience.

Mater, B. D. and Venayagamoorthy, S. K. (2014). The quest for an unambiguous parameterization of mixing efficiency in stably stratified geophysical flows. Geophysical Research Letters, 41(13):4646-4653.

Mater, B. D., Venayagamoorthy, S. K., St. Laurent, L., and Moum, J. N. (2015). Biases in thorpe-scale estimates of turbulence dissipation. part i: Assessments from large-scale overturns in oceanographic data. Journal of Physical Oceanography, 45(10):2497-2521.

Merrifield, S. T., St. Laurent, L., Owens, B., Thurnherr, A. M., and Toole, J. M. (2016). Enhanced diapycnal diffusivity in intrusive regions of drake passage. $a c$ cepted, Journal of Physical Oceanography.

Monin, A. and Obukhov, A. (1954). Basic laws of turbulent mixing in the surface layer of the atmosphere. Contrib. Geophys. Inst. Acad. Sci. USSR, 151:163-187.

Moum, J. N. (1996). Efficiency of mixing in the main thermocline. Journal of Geophysical Research: Oceans, 101(C5):12057-12069.

Munk, W. and Wunsch, C. (1998). Abyssal recipes ii: energetics of tidal and wind mixing. Deep-Sea Research Part I, 45(12):1977-2010.

Munk, W. H. (1966). Abyssal recipes. In Deep Sea Research and Oceanographic Abstracts, volume 13, pages 707-730. 
Nash, J. D. and Moum, J. N. (1999). Estimating salinity variance dissipation rate from conductivity microstructure measurements. Journal of Atmospheric and Oceanic Technology, pages 263-274.

Nash, J. D. and Moum, J. N. (2002). Microstructure estimates of turbulent salinity flux and the dissipation spectrum of salinity. Journal of Physical Oceanography, $32: 2312-2333$.

Naveira Garabato, A. C., Polzin, K. L., Ferrari, R., Zika, J. D., and Forryan, A. (2016). A microscale view of mixing and overturning across the antarctic circumpolar current. Journal of Physical Oceanography, 46(1):233-254.

Naveira Garabato, A. C., Polzin, K. L., King, B. A., Heywood, K. J., and Visbeck, M. (2004). Widespread intense turbulent mixing in the Southern Ocean. Science, 303(5655):210-213.

Nikurashin, M. and Ferrari, R. (2010). Radiation and dissipation of internal waves generated by geostrophic motions impinging on small-scale topography: Application to the Southern Ocean. Journal of Physical Oceanography, 40(9):2025-2042.

Nikurashin, M. and Vallis, G. (2011). A theory of deep stratification and overturning circulation in the ocean. Journal of Physical Oceanography, 41(3):485-502.

Nilsson, E. O., Rutgersson, A., Smedman, A.-S., and Sullivan, P. P. (2012). Convective boundary-layer structure in the presence of wind-following swell. Quarterly Journal of the Royal Meteorological Society, 138(667):1476-1489.

Oakey, N. (1982). Determination of the rate of dissipation of turbulent energy from simultaneous temperature and velocity shear microstructure measurements. Journal of Physical Oceanography, 12(3):256-271.

Obukhov, A. (1946). Turbulence in thermally inhomogeneous atmosphere. Trudy Inst. Teor. Geofiz. Akad. Nauk SSSR, 1:951-115.

Orsi, A. H., Whitworth, T., and Nowlin, W. D. (1995). On the meridional extent and fronts of the Antarctic Circumpolar Current. Deep Sea Research Part I: Oceanographic Research Papers, 42(5):641-673.

Osborn, T. (1980). Estimates of the local rate of vertical diffusion from dissipation measurements. Journal of Physical Oceanography, 10(1):83-89.

Osborn, T. R. (1974). Vertical profiling of velocity microstructure. Journal of Physical Oceanography, 4(1):109-115.

Osborn, T. R. and Cox, C. S. (1972). Oceanic fine structure. Geophysical \& Astrophysical Fluid Dynamics, 3(1):321-345.

Plueddemann, A. and Farrar, J. (2006). Observations and models of the energy flux from the wind to mixed-layer inertial currents. Deep Sea Research Part II: Topical Studies in Oceanography, 53:5 - 30. 
Pollard, R. T. and Millard, R. C. (1970). Comparison between observed and simulated wind-generated inertial oscillations. In Deep Sea Research and Oceanographic Abstracts, volume 17, pages 813-821.

Polzin, K., Kunze, E., Hummon, J., and Firing, E. (2002). The finescale response of lowered adcp velocity profiles. Journal of Atmospheric and Oceanic Technology, $19(2): 205-224$.

Polzin, K. L. and Firing, E. (1997). Estimates of diapycnal mixing using ladep and ctd data from i8s. International WOCE Newsletter, 29:39-42.

Polzin, K. L., Naveira Garabato, A. C., Huussen, T. N., Sloyan, B. M., and Waterman, S. (2014). Finescale parameterizations of turbulent dissipation. Journal of Geophysical Research: Oceans, 119(2):1383-1419.

Polzin, K. L., Toole, J. M., and Schmitt, R. W. (1995). Finescale parameterizations of turbulent dissipation. Journal of Physical Oceanography, 25(3):306-328.

Price, J. F. (1981). Upper ocean response to a hurricane. Journal of Physical Oceanography, 11(2):153-175.

Rath, W., Greatbatch, R. J., and Zhai, X. (2014). On the spatial and temporal distribution of near-inertial energy in the southern ocean. Journal of Geophysical Research: Oceans, 119(1):359-376.

Sallée, J.-B., Shuckburgh, E., Bruneau, N., Meijers, A., Bracegirdle, T., and Wang, Z. (2013). Assessment of southern ocean mixed-layer depths in cmip5 models: Historical bias and forcing response. Journal of Geophysical Research: Oceans, 118(4):1845-1862.

Sanford, T. B., Drever, R. G., Dunlap, J. H., and D'Asaro, E. A. (1982). Design, operation and performance of an expendable temperature and velocity profiler (xtvp). Technical report, DTIC Document.

Schmitt, R. W. (1979). The growth rate of super-critical salt fingers. Deep Sea Research Part A. Oceanographic Research Papers, 26(1):23-40.

Schmitt, R. W. (1994). Double diffusion in oceanography. Annual Review of Fluid Mechanics, 26(1):255-285.

Schmitt, R. W., Toole, J. M., Koehler, R. L., Mellinger, E. C., and Doherty, K. W. (1988). The development of a fine-and microstructure profiler. Journal of Atmospheric and Oceanic Technology, 5(4):484-500.

Schulz, E., Josey, S., and Verein, R. (2012). First air-sea flux mooring measurements in the southern ocean. Geophysical Research Letters, 39(16). 
Sheen, K., Garabato, A. N., Brearley, J., Meredith, M., Polzin, K., Smeed, D., Forryan, A., King, B., Sallée, J., Laurent, L. S., et al. (2014). Eddy-induced variability in Southern Ocean abyssal mixing on climatic timescales. Nature Geoscience, $7(8): 577-582$.

Shih, L. H., Koseff, J. R., Ivey, G. N., and Ferziger, J. H. (2005). Parameterization of turbulent fluxes and scales using homogeneous sheared stably stratified turbulence simulations. Journal of Fluid Mechanics, 525:193-214.

Silverthorne, K. E. and Toole, J. M. (2009). Seasonal kinetic energy variability of near-inertial motions. Journal of Physical Oceanography, 39(4):1035-1049.

Simmons, H. L. and Alford, M. H. (2012). Simulating the long-range swell of internal waves generated by ocean storms. Oceanography.

Sloyan, B. M. (2005). Spatial variability of mixing in the southern ocean. Geophysical research letters, 32(18):L18603.

Sloyan, B. M. and Rintoul, S. R. (2001). The southern ocean limb of the global deep overturning circulation*. Journal of Physical Oceanography, 31(1):143-173.

Sloyan, B. M., Talley, L. D., Chereskin, T. K., Fine, R., and Holte, J. (2010). Antarctic Intermediate Water and Subantarctic Mode Water Formation in the Southeast Pacific: The Role of Turbulent Mixing. Journal of Physical Oceanography, 40(7):1558-1574.

Smith, W. H. and Sandwell, D. T. (1997). Global sea floor topography from satellite altimetry and ship depth soundings. Science, 277(5334):1956-1962.

St. Laurent, L., Naveira Garabato, A. C., Ledwell, J. R., Thurnherr, A. M., Toole, J. M., and Watson, A. J. (2012). Turbulence and Diapycnal Mixing in Drake Passage. Journal of Physical Oceanography, 42(12):2143-2152.

St. Laurent, L. and Schmitt, R. W. (1999). The contribution of salt fingers to vertical mixing in the north atlantic tracer release experiment*. Journal of Physical Oceanography, 29(7):1404-1424.

St. Laurent, L. and Simmons, H. (2006). Estimates of power consumed by mixing in the ocean interior. Journal of climate, 19(19):4877-4890.

Stern, M. E. (1967). Lateral mixing of water masses. In Deep Sea Research and Oceanographic Abstracts, volume 14, pages 747-753.

Stern, M. E., Radko, T., and Simeonov, J. (2001). Salt fingers in an unbounded thermocline. Journal of Marine Research, 59(3):355-390.

Stommel, H. and Arons, A. (1960). On the abyssal circulation of the world ocean-i. stationary planetary flow patterns on a sphere. Deep Sea Research (1953), 6:140154. 
Sun, O. M., Jayne, S. R., Polzin, K. L., Rahter, B. A., and St. Laurent, L. C. (2013). Scaling turbulent dissipation in the transition layer. Journal of Physical Oceanography, 43(11):2475-2489.

Sutherland, G., Christensen, K., and Ward, B. (2014). Evaluating langmuir turbulence parameterizations in the ocean surface boundary layer. Journal of Geophysical Research: Oceans, 119(3):1899-1910.

Terray, E., Donelan, M., Agrawal, Y., Drennan, W., Kahma, K., Williams, A., Hwang, P., and Kitaigorodskii, S. (1996). Estimates of kinetic energy dissipation under breaking waves. Journal of Physical Oceanography, 26(5):792-807.

Thomas, L. and Taylor, J. (2010). Reduction of the usable wind-work on the general circulation by forced symmetric instability. Geophysical Research Letters, 37(18):L18606.

Thomas, L. N., Taylor, J. R., Ferrari, R., and Joyce, T. M. (2013). Symmetric instability in the gulf stream. Deep Sea Research Part II: Topical Studies in Oceanography, 91:96-110.

Thompson, A. F., Gille, S. T., MacKinnon, J. A., and Sprintall, J. (2007). Spatial and temporal patterns of small-scale mixing in Drake Passage. Journal of Physical Oceanography, 37(3):572-592.

Thompson, A. F., Lazar, A., Buckingham, C., Naveira Garabato, A. C., Damerell, G. M., and Heywood, K. J. (2016). Open-ocean submesoscale motions: A full seasonal cycle of mixed layer instabilities from gliders. Journal of Physical Oceanography, (2016).

Thorpe, S. (1977). Turbulence and mixing in a Scottish loch. Philosophical Transactions for the Royal Society of London. Series A, Mathematical and Physical Sciences, pages 125-181.

Thorpe, S. (2004). Langmuir circulation. Annu. Rev. Fluid Mech., 36:55-79.

Thurnherr, A. M., Kunze, E., Toole, J. M., St. Laurent, L., Richards, K. J., and Ruiz-Angulo, A. (2015). Vertical kinetic energy and turbulent dissipation in the ocean. Geophysical Research Letters, 42(18):7639-7647.

Todd, R. E., Owens, W. B., and Rudnick, D. L. (2016). Potential vorticity structure in the north atlantic western boundary current from underwater glider observations. Journal of Physical Oceanography, 46(1):327-348.

Toggweiler, J. and Samuels, B. (1998). On the ocean's large-scale circulation near the limit of no vertical mixing. Journal of Physical Oceanography, 28(9):1832-1852.

Toole, J. M. and Georgi, D. T. (1981). On the dynamics and effects of doublediffusively driven intrusions. Progress in Oceanography, 10(2):123-145. 
Watanabe, M. and Hibiya, T. (2002). Global estimates of the wind-induced energy flux to inertial motions in the surface mixed layer. Geophysical Research Letters, $29(8)$.

Waterman, S., Garabato, A. C. N., and Polzin, K. L. (2013). Internal waves and turbulence in the antarctic circumpolar current. Journal of Physical Oceanography, $43(2): 259-282$.

Waterman, S., Polzin, K. L., Naveira Garabato, A. C., Sheen, K. L., and Forryan, A. (2014). Suppression of internal wave breaking in the Antarctic Circumpolar Current near topography. Journal of Physical Oceanography, 44(5):1466-1492.

Webb, D. and Suginohara, N. (2001). Oceanography: Vertical mixing in the ocean. Nature, 409(6816):37-37.

Weller, R. A. (1982). The relation of near-inertial motions observed in the mixed layer during the jasin (1978) experiment to the local wind stress and to the quasigeostrophic flow field. Journal of Physical Oceanography, 12(10):1122-1136.

Whalen, C., Talley, L., and MacKinnon, J. (2012). Spatial and temporal variability of global ocean mixing inferred from Argo profiles. Geophysical Research Letters, $39(18)$.

Wijesekera, H., Padman, L., Dillon, T., Levine, M., Paulson, C., and Pinkel, R. (1993). The application of internal-wave dissipation models to a region of strong mixing. Journal of Physical Oceanography, 23(2):269-286.

Winters, K. B. and D'Asaro, E. A. (1996). Diascalar flux and the rate of fluid mixing. Journal of Fluid Mechanics, 317:179-193.

Wolfe, C. L. and Cessi, P. (2010). What sets the strength of the middepth stratification and overturning circulation in eddying ocean models? Journal of Physical Oceanography, 40(7):1520-1538.

Wu, L., Jing, Z., Riser, S., and Visbeck, M. (2011). Seasonal and spatial variations of Southern Ocean diapycnal mixing from Argo profiling floats. Nature Geoscience, 4(6):363-366.

Wunsch, C. and Ferrari, R. (2004). Vertical mixing, energy, and the general circulation of the oceans. Annu. Rev. Fluid Mech., 36:281-314.

You, Y. (2002). A global ocean climatological atlas of the turner angle: implications for double-diffusion and water-mass structure. Deep Sea Research Part I: Oceanographic Research Papers, 49(11):2075 - 2093.

Zhai, X., Greatbatch, R. J., Eden, C., and Hibiya, T. (2009). On the loss of windinduced near-inertial energy to turbulent mixing on the loss of wind-onon the loss of wind-induced near-inertial energy to turbulent mixing in the upper ocean. Journal of Physical Oceanography, 39. 